\title{
Ultrasound imaging to tailor treatment of shoulder pain in general practice
}

Citation for published version (APA):

Ottenheijm, R. P. G. (2015). Ultrasound imaging to tailor treatment of shoulder pain in general practice. [Doctoral Thesis, Maastricht University]. Datawyse / Universitaire Pers Maastricht. https://doi.org/10.26481/dis.20151119ro

Document status and date:

Published: 01/01/2015

DOI:

10.26481/dis.20151119ro

Document Version:

Publisher's PDF, also known as Version of record

\section{Please check the document version of this publication:}

- A submitted manuscript is the version of the article upon submission and before peer-review. There can be important differences between the submitted version and the official published version of record.

People interested in the research are advised to contact the author for the final version of the publication, or visit the DOI to the publisher's website.

- The final author version and the galley proof are versions of the publication after peer review.

- The final published version features the final layout of the paper including the volume, issue and page numbers.

Link to publication

\footnotetext{
General rights rights.

- You may freely distribute the URL identifying the publication in the public portal. please follow below link for the End User Agreement:

www.umlib.nl/taverne-license

Take down policy

If you believe that this document breaches copyright please contact us at:

repository@maastrichtuniversity.nl

providing details and we will investigate your claim.
}

Copyright and moral rights for the publications made accessible in the public portal are retained by the authors and/or other copyright owners and it is a condition of accessing publications that users recognise and abide by the legal requirements associated with these

- Users may download and print one copy of any publication from the public portal for the purpose of private study or research.

- You may not further distribute the material or use it for any profit-making activity or commercial gain

If the publication is distributed under the terms of Article $25 \mathrm{fa}$ of the Dutch Copyright Act, indicated by the "Taverne" license above, 


\section{Ultirasound innaging to tailor treatinnent of shoulder pain in general practice}

Ramon Ottenheijm
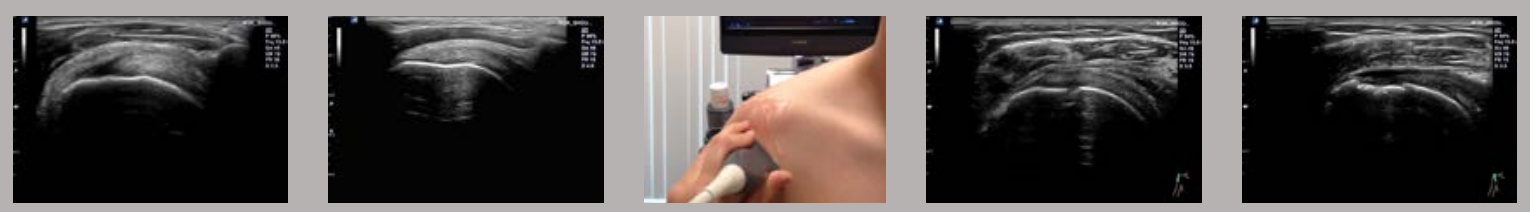
Copyright (C) Ramon PG Ottenheijm, Maastricht 2015

Design logo: Daphne Adams (Canon Nederland)

Design cover: Datawyse

Layout: $\quad$ Tiny Wouters \& Ramon PG Ottenheijm

Printed by: Datawyse | Universitaire Pers Maastricht

ISBN: 978-94-6159-336-8

The research presented in this thesis was conducted at the School for Public Health and Primary Care (CAPHRI), Department of Family Medicine, Maastricht University. CAPHRI participates in the Netherlands School of Primary Care Research (CaRe), acknowledged by the Royal Dutch Academy of Science (KNAW). CAPHRI was classified as 'excellent' by the external evaluation committee of leading international experts that reviewed CAPHRI in December 2010.

Financial support for printing of this thesis was kindly provided by: Medical Coordination Centre Omnes and Dynamic. 


\title{
Ultrasound imaging to tailor treatment of shoulder pain in general practice
}

\author{
PROEFSCHRIFT \\ ter verkrijging van de graad van doctor aan de Universiteit Maastricht, \\ op gezag van de Rector Magnificus, Prof. dr. L.L.G. Soete, \\ volgens het besluit van het College van Decanen, \\ in het openbaar te verdedigen, \\ op donderdag 19 november 2015 om 14.00 uur \\ door \\ Ramon Pieter Georg Ottenheijm
}

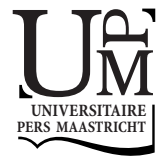




\section{Promotores}

Prof. dr. G.J. Dinant

Prof. dr. R.A. de Bie

\section{Copromotor}

Dr. J.W.L. Cals

\section{Beoordelingscommissie}

Prof. dr. L.W. van Rhijn (voorzitter)

Prof. dr. P.J.E. Bindels (Erasmus Medisch Centrum Rotterdam)

Prof. dr. N.L.U. van Meeteren

Dr. M.J.C.M. Rutten (Jeroen Bosch Ziekenhuis Den Bosch)

Financial support for the MUST-study in this thesis was provided by Medical Coordination Centre Omnes (http://www.mcc-omnes.nl). 


\section{Contents}

Chapter 1 General introduction

Chapter 2 Accuracy of diagnostic ultrasound in patients with suspected subacromial disorders: a systematic review and meta-analysis Arch Phys Med Rehabil 2010; 91:1616-25

Erratum in: Arch Phys Med Rehabil 2010; 91:1962-3

Chapter 3 GPs' perspectives on the diagnostic work-up in patients with shoulder pain: a qualitative study

J Clin Eval Pract 2014; 20:239-45

Chapter 4 Ultrasound diagnosed disorders in shoulder patients in daily general practice: a retrospective observational study BMC Fam Pract 2014;15:115

Chapter 5 The Maastricht Ultrasound Shoulder pain trial (MUST): Ultrasound 69 imaging as a diagnostic triage tool to improve management of patients with non-chronic shoulder pain in primary care BMC Musculoskelet Disord 2011;12:154

Chapter $6 \quad$ Ultrasound imaging for tailored treatment of patients with acute shoulder pain Ann Fam Med 2015;13:53-55

Chapter 7 Clinical effectiveness of ultrasound tailored treatment in patients with acute shoulder pain in general practice: a pragmatic randomised controlled trial (Maastricht Ultrasound Shoulder pain Trial)

Submitted

Chapter 8 General discussion

Summary

Samenvatting

Valorisation

List of publications

Dankwoord

Curriculum vitae 



\section{1}

General introduction 
Chapter 1 


\section{General introduction}

Shoulder pain is defined as pain in the deltoid and upper arm region, often accompanied by restriction in movements. Due to these complaints patients may be limited in activities of daily living, hobbies and sports, especially in tasks which involve above shoulder height action. Also lying on the shoulder can cause pain resulting in problems with sleeping. In this thesis, shoulder pain is defined as acute if it lasts for less than three months.

\section{What is already known?}

\section{The magnitude of the problem}

General practitioners (GPs) are frequently consulted by patients with shoulder pain. In the Netherlands, the annual incidence of shoulder pain in general practice is $29.5 / 1000$ person years. ${ }^{1,2}$ Reported 12 month prevalence varies between 4.7 and $46.7 \%$, while lifetime prevalence is reported up to $67 \% .^{2-4}$ Most studies tend to show a lower prevalence among men than among women. Approximately $40 \%$ of patients with shoulder pain consult their GP, while the remaining persons do not seek medical care. ${ }^{5}$ After initial presentation to the GP, re-consultation takes place in $50 \%$ of the cases. ${ }^{2}$ In the Netherlands, GPs refer $38 \%$ of the cases to either physiotherapy (84\%) or to secondary care (16\%). ${ }^{6}$ The prognosis of shoulder pain is rather poor in many patients. About $50 \%$ of all patients who visit their GP with a new episode of shoulder pain report persistent complaints after six months, and up to $40 \%$ still have pain and functional impairments after 12 months. ${ }^{7,8}$ Forty-six percent of the patients report previous episodes of shoulder pain, and recurrence rates vary from $31 \%$ after 12 months to $85 \%$ after 12 years. $^{9-11}$

Troublesome pain is most common in adult patients with shoulder pain until the age of 65 years. $^{12}$ Roughly $30 \%$ of the patients report limitations in daily life and sick leave is common. $^{5}$

Musculoskeletal disorders are the second most expensive disease group for health costs in the Netherlands. ${ }^{13}$ In 2011 total healthcare costs for musculoskeletal disorders in the Netherlands was $€ 5.2$ billion, which was approximately $5.8 \%$ of total healthcare costs, and $€ 1.1$ billion (21\%) can be attributed to primary care. ${ }^{14}$ It is not known which part can be attributed to shoulder pain. Although shoulder pain related costs during 6 months after presentation do not seem alarmingly high (€689 per patient), it is suggested that prolonged and recurrent episodes generate additional costs for 
expensive care and sick leave. ${ }^{15}$ In general, costs due to sick leave (productivity losses) are substantial and represent a higher burden to the economy than healthcare costs. ${ }^{16}$

\section{Etiology of subacromial disorders}

Disorders of the rotator cuff, including subacromial-subdeltoid bursitis, are the most common cause of shoulder pain seen by GPs. ${ }^{17}$ In the seventies, Neer pointed out that these specific subacromial disorders could not be distinguished by physical examination and radiographic findings. As a result, the term 'impingement syndrome' has been coined to refer to the full range of disorders. ${ }^{18}$ The subacromial structures are prone to develop pathology, and the spectrum of pathology is extensive and includes tendinopathy (tendinosis), calcific tendonitis, partial- or full-thickness tears in one or more of the rotator cuff muscles, and subacromial-subdeltoid bursitis. ${ }^{19}$ The exact mechanism for development of these subacromial disorders is still unclear. Several theories have been hypothesized to explain the mechanisms leading to pathology. Since Neers' publications in 1971 and 1983, it was accepted that biomechanical factors, mainly compression of the rotator cuff tendons beneath the acromion (impingement), were most prominent in the development of shoulder pain. ${ }^{18}$ This resulted in the introduction of the aforementioned term 'impingement syndrome'. However, improving diagnostic imaging techniques and arthroscopy showed that this hypothesis could no longer be underpinned. Nowadays, there is growing evidence that the etiology of rotator cuff disease is likely a complex of conditions comprising intrinsic and extrinsic factors. Both intrinsic failure of the tendon and extrinsic factors play an important role. Known intrinsic processes are of genetic, vascular and degenerative origin. Repetitive stress and overhead movements are extrinsic factors that can lead to subacromial disorders. Also, traumatic incidents like falling on the arm and applying excessive force like heavy lifting can lead to tears. ${ }^{20-23}$

\section{How do GPs manage patients with shoulder pain?}

\section{Diagnosis}

In 1990, the Dutch College of General Practitioners issued the first guidelines for the management of shoulder pain. GPs were advised to use a diagnostic classification which was largely based on the concepts of Cyriax. According to Cyriax, a systematic physical examination enables physicians to reach a specific, patho-anatomical diagnosis, e.g. supraspinatus tendinitis or acute bursitis. ${ }^{24}$ However, in the revised guidelines issued in 1999 and 2008, GPs are advised to refrain from using this 
diagnostic Cyriax classification to diagnose shoulder pain. ${ }^{17}$ This advice was based on research that illustrated the lack of interobserver agreement on the appropriate diagnostic criteria and various classifications used for the diagnostic interpretation. ${ }^{25-27}$ Also, it became clear that distinct groups of patients can be obtained from medical history and physical examination data, patients with limitations in range of motion and those with pain without limitations in range of motion, but that the distinction between the groups in no way mirrors Cyriax's classification. ${ }^{26}$ In summary, physical examinations used to evaluate the various disorders are fraught with uncertainty. As a result, the diagnostic phase does not often lead to a specific, patho-anatomical diagnosis. Therefore, the current shoulder pain guidelines issued in 2008 proposes a non-specific classification based on pain and physical examination findings, which can be linked to the origin of the complaints (e.g. subacromial or a specific joint). ${ }^{17}$

\section{Current treatment}

In The Netherlands, every Dutch resident is registered with a GP. Patients first visit their GP before visiting a specialist in secondary care. This is comparable with, for instance, the British healthcare system. Management of shoulder pain is primarily done by GPs. Almost $90 \%$ of patients with shoulder pain are at first visit diagnosed and treated in general practice, while only $10 \%$ is being referred for a specialist opinion. ${ }^{7}$ Usual care according to the latest shoulder pain guidelines consists of providing information, treatment and lifestyle recommendations, medication and referral to physiotherapy and secondary care. As pointed out, GPs are advised to start treatment based on patients' signs and symptoms rather than on a suspected specific diagnosis. The advised treatment for all patients consists of a stepwise approach, which starts with advice and paracetamol or NSAIDs for 2 weeks. In persisting cases corticosteroid injections into the subacromial bursa or glenohumeral joint and referral to a physiotherapist are advised, depending on the level and origin of pain and functional limitations respectively. Referral to secondary care or for additional diagnostic tests are advised if these usual care treatments fail or in case of the suspicion of severe disorders, e.g. rheumatoid arthritis or cancer (red flag situations). ${ }^{17}$ In conclusion, one can say that the current shoulder pain guidelines do not provide GPs with tailor made treatment strategies. 


\section{What is the current role of ultrasound imaging?}

In cases where physical examination cannot differentiate, ultrasound imaging can be a useful imaging technique to detect subacromial disorders (examples of ultrasound imaging of subacromial disorders are provided in Figure 1.1). ${ }^{28-32}$ Recent meta-analyses and studies evaluating the interrater-reliability show that radiologists are able to accurately diagnose rotator cuff tears. In detecting full-thickness rotator cuff tears, ultrasound imaging showed excellent diagnostic accuracy, ${ }^{29,33}$ and an almost perfect interrater-reliability (overall kappa ranging from 0.90 - to 0.95 ). ${ }^{34,35}$ In detection of partial-thickness rotator cuff tears, specificity remains high, but sensitivity decreases (0.67 to 0.73$),{ }^{29,33}$ with a substantial interrater-reliability (overall kappa ranging from 0.63 to 0.79$).{ }^{34,35}$ Also, the remaining subacromial disorders tendinopathy, calcific tendonitis, and subacromial-subdeltoid bursitis can be visualized. ${ }^{36}$ However, diagnostic accuracy of these latter disorders remains unclear. The current shoulder pain guidelines advise GPs to refrain from additional diagnostic imaging techniques as ultrasound imaging in patients with acute shoulder pain. ${ }^{17}$ This advice is based on the assumption that imaging results will not change currently advised usual care, and that many disorders are also common in asymptomatic populations. As a result, additional imaging can lead to overdiagnosis, and subsequently unnecessary referrals to secondary care. ${ }^{17}$ Ultrasound imaging is advised in patients in whom conservative treatment fails and in red flag situations. Remarkably, Dutch GPs tend to refrain from this advice and increasingly order ultrasound imaging in patients with acute shoulder pain (unpublished data), which is also the case in Australia. ${ }^{37-39}$

\section{How to improve management?}

Given the poor outcome, we can conclude that effective treatment for shoulder pain in general practice can be improved. One can say that patients with shoulder pain, without sufficient treatment, are likely to be symptomatic for a long time. As pointed out above, GPs are not able to distinguish between different subacromial disorders. The advised treatment for all patients, regardless of the underlying diagnosis, consists of a stepwise approach: when the initial applied treatment is unsuccessful, other interventions are considered. The current diagnostic process leads to a substantial case mix, and the stepwise treatment approach is expected to dilute the effects of the indicated interventions in the total population considerably. Interestingly, for all specific subacromial disorders, specific therapies are available ${ }^{40,41}$ 


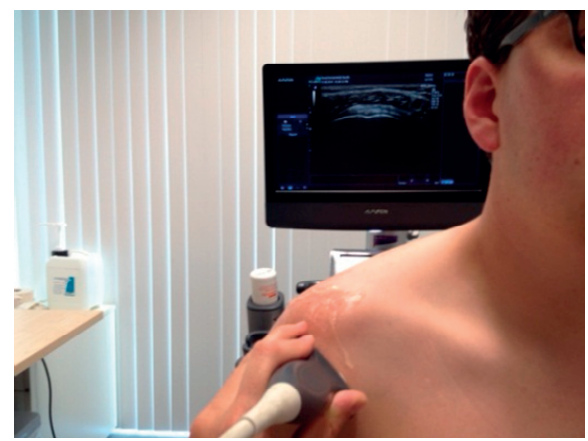

Ultrasound imaging right shoulder Longitudinal view of supraspinatus tendon

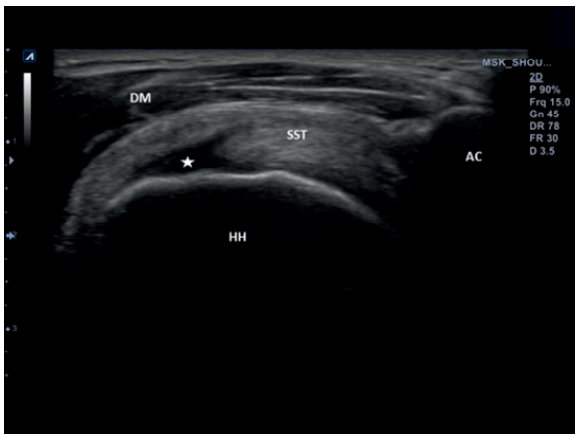

Partial-thickness tear

$\mathrm{AC}$, Acromion; $\mathrm{HH}$, humeral head; DM, deltoid muscle; SST, supraspinatus tendon; Asterisk, partial-thickness tear

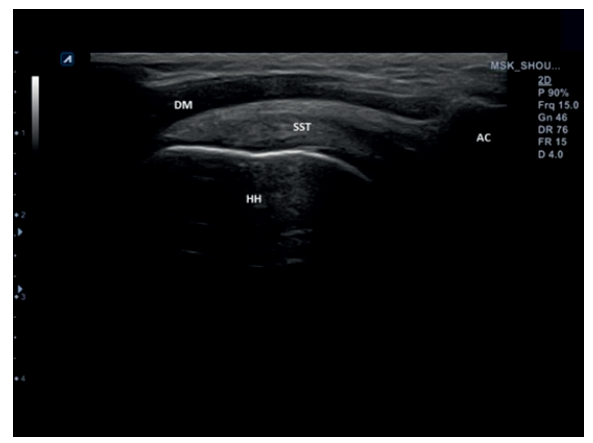

Normal tendon

AC, Acromion; $\mathrm{HH}$, humeral head; DM, deltoid muscle; SST, supraspinatus tendon

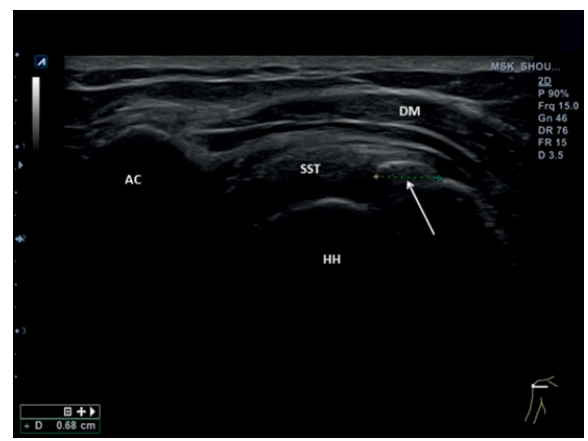

Calcific tendonitis

AC, Acromion; $\mathrm{HH}$, humeral head; DM, deltoid muscle; SST, supraspinatus tendon; Arrow, calcium deposit

Figure 1.1 Ultrasound imaging of the supraspinatus tendon

It sounds likely that an accurate diagnosis is important in order to minimise the social and economic impact. A delayed or incorrect diagnosis exposes patients and the healthcare system to the burden of delayed specific therapy tailored to the underlying diagnosis, subsequently leading to unnecessary interventions and referrals. Moreover, time spent in an unsuccessful intervention delays a possibly more appropriate intervention. This increases the likelihood of the development of chronic pain and reduces the effectiveness of future interventions. For example, in patients with tendinopathy the benefit of a corticosteroid injection is debatable; what is the advantage if there is no inflammation and tendon quality might deteriorate? Physiotherapists can use diagnostic information from ultrasound imaging to apply exercise programs avoiding strength training, as overuse of degenerative tendons can 
lead to tearing. Also, a delay in tear repair can result in irreparable tears because of significant tendon retraction or muscle atrophy. ${ }^{42}$ In order to improve treatment and prognosis of patients with shoulder pain, a more specific diagnosis at the earliest possible stage seems necessary.

Due to the sufficient diagnostic accuracy and clear relationship between ultrasound diagnosis and available evidence based therapies, combining clinical information with ultrasound diagnosis is potentially helpful to tailor treatment in patients with shoulder pain. Therefore, we hypothesise that the currently advocated stepwise approach in the guidelines can be improved by tailoring treatment to the ultrasound diagnosis in patients with acute shoulder pain. The effectiveness of this test-treatment combination, which has not been studied before, is the mainstay of this thesis. This thesis foresees in the evidence gap that is addressed in the shoulder pain guidelines of the Dutch College of General Practitioners.

\section{Objectives of this thesis}

The primary objective of this thesis is to investigate the effects of tailoring treatment to an ultrasound diagnosis on clinical recovery, compared to the current standard of care for patients with acute shoulder pain (pain less than 3 months), who present in general practice. To achieve this objective, other adjacent objectives need to be studied. With each objective we try to bridge evidence gaps in the management of shoulder pain in general practice.

\section{Translation into research questions}

On the basis of our objectives, we formulated the following research questions for this thesis.

Q1 What is the diagnostic accuracy of ultrasound imaging to detect subacromial disorders in patients presenting in primary and secondary care settings?

Q2 Which perspectives do GPs have on the diagnostic work-up of patients with shoulder pain?

Q3 What are the frequencies of specific findings as diagnosed with ultrasound imaging in patients with shoulder pain and their possible predictors?

Q4 What is the clinical effectiveness of tailoring treatment to an ultrasound diagnosis, compared to the current standard of care for patients with acute shoulder pain in general practice? 


\section{Outline of this thesis}

Following this general introduction, a systematic review and meta-analysis is presented in Chapter 2 with the goal to evaluate the diagnostic accuracy of ultrasound imaging to detect the full spectrum of subacromial disorders (Q1). So far, only studies that have reviewed the diagnostic accuracy of ultrasound imaging for the diagnosis of rotator cuff tears have been published.

Despite the pragmatic diagnostic work-up of patients with shoulder pain as advocated in the shoulder guideline, this diagnostic work-up is generally experienced as complex. Chapter $\mathbf{3}$ is a qualitative study, where GPs were interviewed about their perspectives on the diagnostic work-up of patients with shoulder pain (Q2). Topics of the interviews were how GPs diagnose patients with shoulder pain, what diagnostic classifications do GPs use, which barriers in the diagnostic work-up do GPs face and the role of imaging and ultrasound imaging in particular.

We know that GPs increasingly order ultrasound imaging in patients with shoulder pain. However, little is known about the diagnostic yield of ultrasound imaging. In Chapter 4 we describe the specific shoulder disorders detected on ultrasound imaging in general practice patients who were symptomatic enough and have had symptoms for an adequate duration to warrant referral by their GP for ultrasound imaging (Q3).

The study protocol of the Maastricht Ultrasound Shoulder pain Trial, a pragmatic, randomised controlled multicentre trial assessing the clinical effectiveness of tailoring treatment to an ultrasound diagnosis in patients with acute shoulder pain, who present in general practice, is presented in Chapter 5. In this protocol we describe the design and methods for Q3 and Q4.

Without knowledge of the prevalence of the ultrasound imaging encountered disorders, it is debatable if tailored treatment is possible. Therefore, we present the ultrasound findings of patients with acute shoulder pain, enrolled in the clinical trial, and their possible predictors in Chapter 6 (Q3).

In Chapter 7 we present the clinical and health related quality of life outcomes of treatment tailored to ultrasound diagnosis (Q4). In our trial, treatment of patients with acute shoulder pain was tailored to the specific disorder observed by ultrasound imaging.

Finally, this thesis concludes in Chapter $\mathbf{8}$ with a general discussion with elaboration on methodological considerations of the trial, and discussion of the findings of the previous chapters, while offering possible implications for clinical practice and future research. 


\section{References}

1. Feleus A, Bierma-Zeinstra SMA, Miedema HS, Bernsen RMD, Verhaar JAN, Koes BW. Incidence of nontraumatic complaints of arm, neck and shoulder in general practice. Man Ther. 2008;13:426-433.

2. Greving K, Dorrestijn O, Winters JC, et al. Incidence, prevalence, and consultation rates of shoulder complaints in general practice. Scan J Rheumatol. 2012;41:150-155.

3. Luime JJ, Koes BW, Hendriksen IJM, et al. Prevalence and incidence of shoulder pain in the general population; a systematic review. Scan J Rheumatol. 2004;33:73-81.

4. Reilingh ML, Kuijpers T, Tanja-Harfterkamp AM, van der Windt DA. Course and prognosis of shoulder symptoms in general practice. Rheumatology. 2008;47:724-730.

5. Picavet HS, Schouten JS. Musculoskeletal pain in the Netherlands: prevalences, consequences and risk groups, the DMC(3)-study. Pain. 2003;102:167-178.

6. Dorrestijn $\mathrm{O}$, Greving $\mathrm{K}$, van der Veen WJ, et al. Patients with shoulder complaints in general practice: consumption of medical care. Rheumatology. 2011;50:389-395.

7. van der Windt DA, Koes BW, Boeke AJ, Devillé W, De Jong BA, Bouter LM. Shoulder disorders in general practice: prognostic indicators of outcome. Br J Gen Pract. 1996;46:519-523.

8. Winters JC, Sobel JS, Groenier KH, Arendzen JH, Meyboom-de Jong B. The long-term course of shoulder complaints: a prospective study in general practice. Rheumatology. 1999;38:160-163.

9. Andersson IH. The course of non-malignant chronic pain: a 12-year follow-up of a cohort from the general population. Eur J Pain. 2004;8:47-53.

10. Macfarlane GJ, Hunt IM, Silman AJ. Predictors of chronic shoulder pain: a population based prospective study. J Rheumatol. 1998;25:1612-1615.

11. Luime JJ, Kuiper JI, Koes BW, Verhaar JAN, Miedema HS, Burdorf A. Work-related risk factors for the incidence and recurrence of shoulder and neck complaints among nursing-home and elderly-care workers. Scand J Work Environ Health. 2004;30:279-286.

12. Parsons S, Breen A, Foster NE, et al. Prevalence and comparative troublesomeness by age of musculoskeletal pain in different body locations. Fam Pract. 2007;24:308-316.

13. Meerding WJ, Bonneux L, Polder JJ, Koopmanschap MA, van der Maas PJ. Demographic and epidemiological determinants of healthcare costs in Netherlands: cost of illness study. BMJ. 1998; 317:111-115.

14. RIVM. Kosten van ziekten tool (Health care costs in the Netherlands 2011). In: Kosten van Ziekten 2011. Bilthoven: RIVM. Available at www.kostenvanziekten.nl

15. Kuijpers T, van Tulder MW, van der Heijden GJMG, Bouter LM, van der Windt DIAWM. Costs of shoulder pain in primary care consulters: a prospective cohort study in The Netherlands. BMC Musculoskelet Disord. 2006;7:83.

16. Jensen MK, Sjogren P, Ekholm O, Rasmussen NK, Eriksen J. Identifying a long-term/chronic, non-cancer pain population using a one-dimensional verbal pain rating scale: an epidemiological study. Eur J Pain. 2004;8:145-152.

17. Winters JC, van der Windt DAWM, Spinnewijn WEM, et al. Shoulder pain guideline of the Dutch College of General Practitioners (in Dutch). Huisarts Wet. 2008;51:555-565.

18. Neer CSn. Impingment lesions. Clin Orthop Rel Res. 1983;173:70-77.

19. Papadonikolakis A, McKenna M, Warme W, Martin BI, Matsen FA, 3rd. Published evidence relevant to the diagnosis of impingement syndrome of the shoulder. J Bone Joint Surg Am. 2011;93:1827-1832.

20. Benson RT, McDonnell SM, Knowles HJ, Rees JL, Carr AJ, Hulley PA. Tendinopathy and tears of the rotator cuff are associated with hypoxia and apoptosis. J Bone Joint Surg. 2010;92:448-453.

21. Hashimoto T, Nobuhara K, Hamada T. Pathologic evidence of degeneration as a primary cause of rotator cuff tear. Clin Orthop Rel Res. 2003:111-120.

22. Khan KM, Cook JL, Maffulli N, Kannus $P$. Where is the pain coming from in tendinopathy?: It may be biochemical, not only structural, in origin. Br J Sports Med. 2000;34:81-83.

23. Lohr JF, Uhthoff HK. The microvascular pattern of the supraspinatus tendon. Clin Orthop Rel Res. 1990:35-38.

24. Cyriax J. Textbook of Ortopedic Medicine. 7th edition: London: Ballière Tindal; 1987. 
25. de Winter AF, Jans MP, Scholten RJ, Deville W, van Schaardenburg D, Bouter LM. Diagnostic classification of shoulder disorders: interobserver agreement and determinants of disagreement. Ann Rheum Dis. 1999;58:272-277.

26. Groenier $\mathrm{KH}$, Winters JC, de Jong BM. Classification of shoulder complaints in general practice by means of nonmetric multidimensional scaling. Arch Phys Med Rehabil. 2003;84:812-817.

27. De Jongh AC. Shoulder complaints in general practice (Dissertation). Rotterdam, Erasmus University Rotterdam; 1994.

28. Allen GM, Wilson DJ. Ultrasound of the shoulder. Eur J Ultrasound. 2001;14:3-9.

29. Dinnes J, Loveman E, Mclntyre L, Waugh N. The effectiveness of diagnostic tests for the assessment of shoulder pain due to soft tissue disorders: a systematic review. Health Technol Assess. 2003;7:1-166.

30. Jacobson JA, van Holsbeeck MT. Musculoskeletal ultrasonography. Orthop Clin North Am. 1998;29: 135-167.

31. Middleton W, Teefey S, Yamaguchi K. Sonography of the rotator cuff: analysis of interobserver variability. Am J Roentgenol. 2004;183:1465-1468.

32. Martinoli C, Bianchi S, Prato N, et al. US of the shoulder: non-rotator cuff disorders. Radiographics. 2003;23:381-401.

33. de Jesus JO, Parker L, Frangos AJ, Nazarian LN. Accuracy of MRI, MR arthrography, and ultrasound in the diagnosis of rotator cuff tears: a meta-analysis. Am J Roentgenol. 2009;192:1701-1707.

34. Le Corroller T, Cohen M, Aswad R, Pauly V, Champsaur P. Sonography of the painful shoulder: role of the operator's experience. Skeletal Radiol. 2008;37:979-986.

35. Rutten MJ, Jager GJ, Kiemeney LA. Ultrasound detection of rotator cuff tears: observer agreement related to increasing experience. Am J Roentgenol. 2010;195:W440-446.

36. Bianchi S, Martinoli C. Ultrasound of the musculoskeletal system. Berlin Heidelberg New York: Springer; 2007.

37. Britt H, Miller GC, Knox S. Shoulder syndrome. In imaging orders by general practitioners in Australia 1999-2000. AlHW Cat. No. GEP 7. General Practice Series No. 7. 2001. Available at: www.aihw.gov.au/publications/index.cfm/title/6949

38. Broadhurst NA, Gialamis A, McElroy HJ, Bielby JJ. How do Australian GPs manage shoulder dysfunction? Aust Fam Physician. 2004;33:861-864.

39. Masters S, O'Doherty L, Mitchell GK, Yelland M. Acute shoulder pain in primary care - an observational study. Aust Fam Physician. 2007;36:473-476.

40. Baring T, Emery RJH, Reilly P. Management of rotator cuff disease: specific treatment for specific disorders. Best Pract Res Clin Rheumatol. 2007;21:279-294.

41. Grant HJ, Arthur A, Pichora DR. Evaluation of interventions for rotator cuff pathology: a systematic review. J Hand Ther. 2004;17:274-299.

42. Yamaguchi K, Ditsios K, Middleton WD, Hildebolt CF, Galatz LM, Teefey SA. The demographic and morphological features of rotator cuff disease. A comparison of asymptomatic and symptomatic shoulders. J Bone Joint Surg Am. 2006;88:1699-1704. 


\section{Chapter}

Accuracy of diagnostic ultrasound in patients with suspected subacromial disorders: a systematic review and meta-analysis

Ramon PG Ottenheijm, Mariëtte J Jansen, J Bart Staal, Ann van den Bruel, René E Weijers, Rob A de Bie, Geert-Jan Dinant Arch Phys Med Rehabil 2010; 91:1616-25 Erratum in: Arch Phys Med Rehabil 2010; 91:1962-3 


\section{ABSTRACT}

\section{Objective}

To determine the diagnostic accuracy of ultrasound for detecting subacromial disorders in patients presenting in primary and secondary care settings.

\section{Data sources}

Medline and Embase were searched on June $9^{\text {th }}, 2010$. In addition, the reference list of one systematic review and all included articles were searched to identify relevant studies.

\section{Study selection}

Two reviewers independently selected the articles evaluating the accuracy of ultrasound for detecting subacromial disorders from the title and abstracts retrieved by the literature search. Selection criteria were ultrasound frequency $\geq 7.5 \mathrm{MHz}$ as index test, surgery, MRI and/or radiography as reference standards, and subacromial disorders as target conditions.

\section{Data extraction}

Two reviewers independently extracted the data on study characteristics and results to construct $2 \times 2$ tables, and performed a methodological quality assessment.

\section{Data synthesis}

Twenty-three studies were included: 22 reported on full-thickness rotator cuff tears, 15 on partial-thickness tears, 3 on subacromial bursitis and 2 on tendinopathy and calcifying tendonitis, respectively. For fullthickness tears, pooled sensitivity of ultrasound was 0.95 ( $95 \% \mathrm{Cl} 0.90$ to 0.97$)$, and specificity 0.96 (0.93 to 0.98). For partial-thickness tears, pooled sensitivity was 0.72 ( 0.58 to 0.83 ), and specificity 0.93 ( 0.89 to 0.96 ). Statistical pooling was not possible for the other disorders. For subacromial bursitis, sensitivity ranged from 0.79 to 0.81 , and specificity from 0.94 to 0.98 . For tendinopathy, sensitivity ranged from 0.67 to 0.93 , specificity from 0.88 to 1.00 . Sensitivity for calcifying tendonitis was 1.00 in both studies, with specificity ranging from 0.85 to 0.98 .

\section{Conclusions}

We strongly recommend ultrasound in patients for whom conservative treatment fails, to rule in or out fullthickness tears, to rule in partial-thickness tears and to a lesser extent to diagnose tendinopathy, subacromial bursitis and calcifying tendonitis. These results can help physicians tailor treatment. 


\section{Introduction}

Shoulder complaints are a common and troublesome problem in primary and secondary care settings, with an unfavourable prognosis. ${ }^{1-3}$ Subacromial disorders are considered to be the most common pathology affecting the shoulder. The spectrum is extensive and includes rotator cuff tendinopathy (tendinosis), calcifying tendonitis, partial- or full-thickness tears, acute or chronic bursitis and bursal reactions. ${ }^{4}$ Conflicting theories have been proposed to explain the mechanisms leading to pathology, and more research is needed to understand the disease process. ${ }^{4}$ However, the dominant view is that many shoulder complaints have their origin in a dynamic pathology, with subacromial impingement as the initial stage, and rotator cuff tears as the final stage. ${ }^{5,6}$ This requires a combined patho-anatomical and dynamic diagnosis.

Management of shoulder complaints involves various medical disciplines, including general practice medicine, physiotherapy, orthopaedic surgery, rheumatology, sports medicine, rehabilitation medicine and radiology. For each of these disciplines, the diagnosis of patients with shoulder complaints is a complex problem. Research shows that physical examinations used to evaluate various disorders are fraught with uncertainty. ${ }^{7-11}$ Nevertheless, an accurate diagnosis is essential to ensure that patients receive appropriate and timely treatment and correct information regarding their prognosis. Unfortunately, the diagnostic phase often does not lead to an adequate patho-anatomical diagnosis. Most patients are therefore managed in a stepwise treatment approach, where response to conservative treatment indicates whether surgical intervention is needed. This is undesirable, as benefits of treatment may vary for different disorders, especially in view of the availability of specific treatments for specific subacromial disorders. ${ }^{12,13}$ For example, patients with a suspected rotator cuff tear preferably are referred to an orthopaedic surgeon to assess surgery options, as positive postoperative outcome has been correlated with early surgical repair, ${ }^{14,15}$ while patients without a surgical indication should not necessarily be seen by an orthopaedic surgeon. Therefore, a plausible first step to achieve more appropriate treatment decisions seems to establish a more accurate diagnosis.

Musculoskeletal ultrasound (US) can be a useful imaging method to detect subacromial disorders. ${ }^{16-21}$ Two studies have reviewed the diagnostic accuracy of US for the diagnosis of rotator cuff tears and showed that, in secondary care settings, US is accurate and the most cost-effective test to identify full-thickness tears, and to a lesser extent also to diagnose partial-thickness tears. ${ }^{17,21}$ The transferability of these results to settings with lower prevalence and different disease spectra remains to be determined. The diagnostic accuracy of US for the full spectrum of subacromial disorders, while distinguishing between primary and secondary care settings, therefore remains unclear. 
The objective of our systematic review was to determine the diagnostic accuracy of US to detect subacromial disorders in patients presenting in primary and secondary care settings.

\section{Methods}

\section{Search strategy}

A well-conducted systematic review on this topic was published in 2003 , using a search strategy comparable to ours. ${ }^{17}$ This review was based on a search from 1985 to October 2001. We updated this review by using its reference list and conducting a new search, incorporating the methods recommended in the Cochrane Handbook on Systematic Reviews of Diagnostic Tests. ${ }^{22}$ Two reviewers (MJ and RO) searched the bibliographic databases of Pubmed and EMBASE from 2001 up to June $9^{\text {th }}, 2010$, with a set of MeSH and key terms for the index test, target conditions, and diagnostic accuracy (see Table 2.1). In addition, references of all included studies were hand-searched for relevant studies.

\section{Selection criteria}

Two reviewers (MJ and RO) independently screened the titles and abstracts for relevance and full papers for inclusion, and extracted data. Disagreements were resolved by consensus. Studies were eligible if they included adults with suspected subacromial disorders diagnosed with subacromial bursitis, or the rotator cuff disorders of tendinopathy, calcifying tendonitis and tears (either partial- or full-thickness). The index test was US imaging using linear array transducers with a frequency of $\geq 7.5 \mathrm{MHz}$. Reference standards used for all target conditions had to consist of MRI, surgery (open or arthroscopic) and/or radiography. The criteria for pathology, scanning technique(s) and/or surgery procedure had to be described in some detail or reference had to be made to established techniques or procedures. Studies were included in the review if they were full reports in English, French, German or Dutch in peer-reviewed journals. We only included test accuracy studies allowing the generation of $2 \times 2$ tables. Authors were contacted if relevant data were lacking. 
Table 2.1 Search strategy

\begin{tabular}{|c|}
\hline Search terms \\
\hline For Pubmed \\
\hline (“Ultrasonography"(MeSH Terms) OR “Ultrasonography” (tw) OR \\
\hline “Echography"(tw) OR “ultrasound" (tw) OR "sonography") \\
\hline AND \\
\hline (“Shoulder"(MesH Terms) OR “shoulder"(tw) OR “Shoulder pain” (MeSH \\
\hline Terms) OR “Shoulder pain"(tw) OR "Shoulder impingement \\
\hline syndrome"(MeSH Terms) OR “Shoulder impingement syndrome"(tw) OR \\
\hline "Bursitis"(MeSH Terms) OR "Bursitis"(tw) OR “Tendinopathy"(MeSH \\
\hline $\begin{array}{l}\text { Terms) OR “Tendinopathy"(tw) OR “Rotator cuff”(MeSH) OR “Rotator } \\
\text { cuff"(tw) OR “tend*”(tw)) }\end{array}$ \\
\hline
\end{tabular}

AND

("Sensitivity and specificity"(MeSH) OR sensitiv*(tw) OR

“Diagnosis"(MeSH) OR “Diagnosis"(tw))

Set

Index test set

Target condition set

Limits: Languages English, French, German and Dutch;

Adults $19-80+$ years

\section{For Embase (Ovid format)}

exp Echography

Index test set

Ultrasonography.mp

Ultrasound.mp.

Sonography.mp.

or/1-4

exp Shoulder

exp Shoulder pain

or/6-7

exp Shoulder impingement syndrome

exp Rotator cuff

exp Tendinitis

Subacromial bursitis.mp.

or/9-12

Sensitivity.mp.

Specificity.mp.

Diagnostic accuracy set

or/14-15

5 and 8 and 13 and 16

Combined sets

Limits: Languages English, French, German and Dutch 


\section{Methodological quality assessment}

The methodological quality of each selected study was assessed independently by two reviewers ( $\mathrm{MJ}$ and $\mathrm{RO}$ ) using the Quadas tool, which highlights the strengths and weaknesses of diagnostic accuracy studies. $^{23}$ Disagreements were resolved by consensus.

\section{Data synthesis}

All eligible studies were first categorized and data analysis was performed according to the target condition. We retrieved sensitivity and specificity data, with their $95 \%$ confidence intervals $(95 \% \mathrm{Cl})$, for each individual study, and plotted these results using Review Manager 5. ${ }^{a}$ The recommended bivariate model, which takes into account the negative correlation between sensitivity and specificity, was used to produce summary estimates of sensitivity, specificity, positive and negative likelihood ratios (LR+ and LR-), with their $95 \% \mathrm{Cl}$. These intervals take into account the heterogeneity beyond chance between studies (random effects model). ${ }^{24,25}$ These parameters were used to obtain hierarchical summary receiver operating characteristic curves (HSROC curves). Potential sources of heterogeneity were investigated by carrying out several predefined subgroup analyses: study design, transducer frequency, age, prevalence and key biases. This statistical analysis and drawing used the Metandi module in STATA $10 .^{b}$

\section{Results}

\section{Study selection and characteristics}

Our search strategy yielded 137 records. Figure 2.1 summarizes the flow of records through the review. Of the 114 excluded records, 18 were duplicates and 56 were rejected on the basis of the title or abstract. Of the remaining records, eight provided insufficient data, and 32 were excluded for other reasons: other populations, other target condition, US not used as index test, US transducer frequencies $<7.5 \mathrm{MHz}$ or arthrography as a reference standard.

Twenty-three studies thus met the selection criteria for inclusion in this review. ${ }^{26-48}$ We found accuracy results for all target conditions; 15 studies evaluated more than one target condition. The outcomes most commonly reported were full-thickness tears (22 studies, 1843 participants, 875 diseased) and partial-thickness tears (15 studies,

\footnotetext{
${ }^{a}$ Review Manager software. Available at: http://www.cc-ims.net/revman

b METANDI: Stata module to perform meta-analysis of diagnostic accuracy. Statistical Software Components 2008. Available at: http://ideas.repec.org/c/boc/bocode/s456932.html
} 
1456 participants, 344 diseased). Accuracy results for subacromial bursitis were presented in three studies (377 participants, 85 diseased), and those for calcifying tendonitis (300 participants, 41 diseased) and tendinopathy (97 participants, 32 diseased) in two studies. Seven of the eight authors contacted about missing data in their articles did not provided additional information. One author provided additional information, which contained insufficient data.

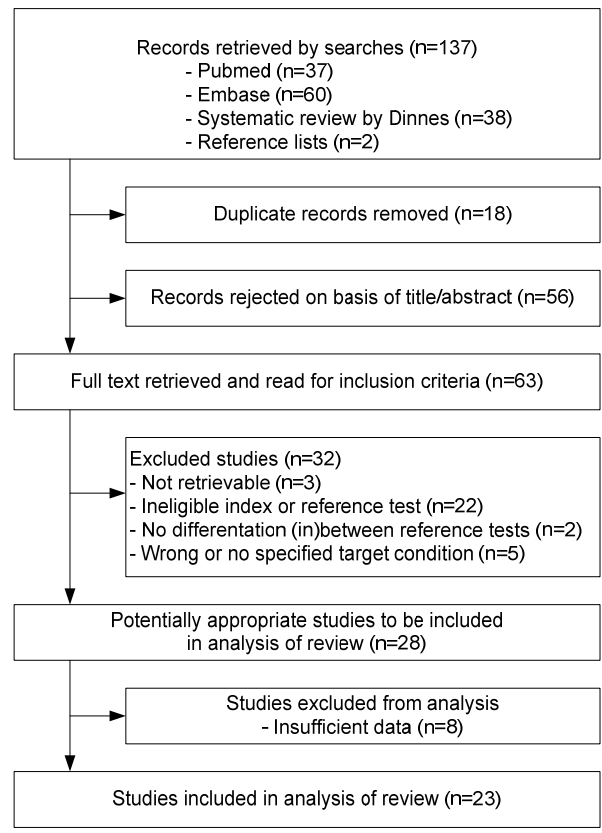

Figure 2.1 Flowchart of the search and selection process.

Table 2.2 shows the characteristics of the studies included. Most studies were conducted at orthopaedic and/or radiology departments; not a single study was conducted in a primary care setting. Across the studies, the mean age of included participants was 52 years. The majority of participants were characterized by having suspected rotator cuff disorders and by failure of conservative treatment. Eleven studies $^{28,29,32,34,35,38,41,44,45,47,48}$ offered no clear presentation of the study design, eight were prospective in design ${ }^{26,31,33,36,39,42,43,46}$ and four had a retrospective design. ${ }^{27,30,37,40}$ The mean sample size was 93, and eight studies included at least 100 participants. The frequencies of the US transducers varied between the studies, with more recent studies ( $n=7$ ) using frequencies $\geq 10 \mathrm{MHz}{ }^{26-28,30,34,40,45}$ Only one study used MRI instead of surgery (open or arthroscopic) as a reference standard. ${ }^{36}$ 


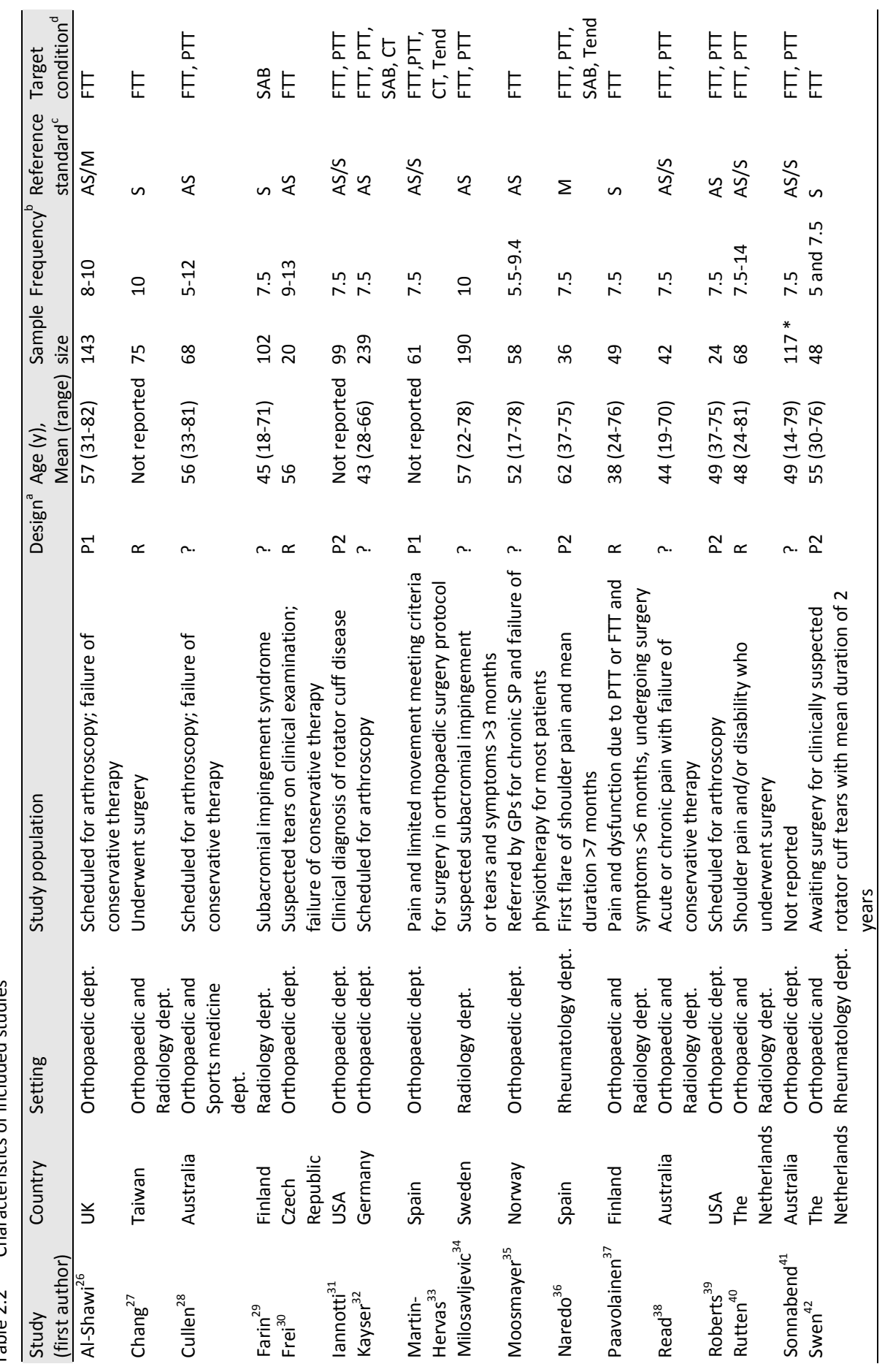




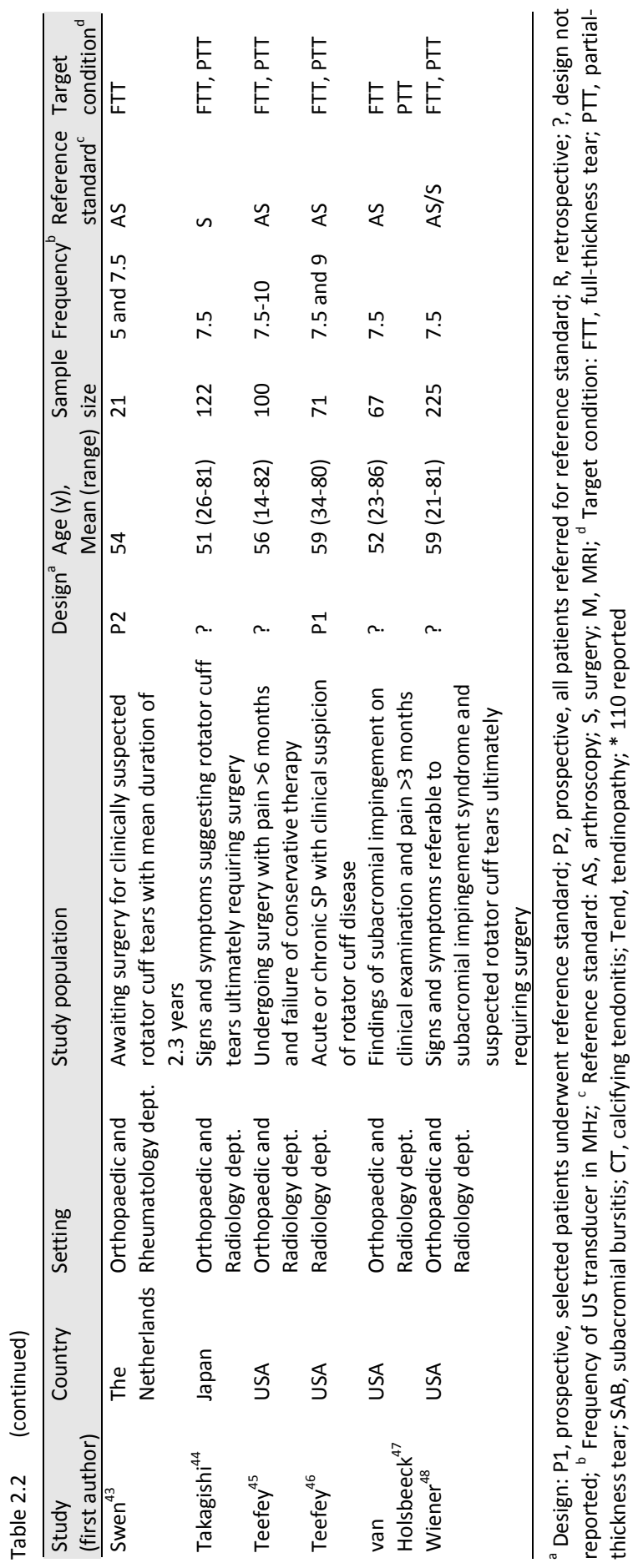




\section{Methodological quality assessment}

Details about the quality assessment are presented in Table 2.3. Nineteen studies $^{26,28,30,31,33-40,42-48}$ provided enough information to evaluate the spectrum of participants included. In nine studies ${ }^{27,28,35,36,38,41,43,45,46}, \leq 3$ months elapsed between US and the reference standard, a time interval we consider to be the maximum to be reasonably sure that the target condition did not change between the two tests. Partial verification (performance of the reference standard depending on US outcome) was clearly avoided in 12 studies. $^{26,30-36,38,42,43,47}$ In nineteen studies, the US results were interpreted without knowledge of the reference standard. Since US is normally performed before the reference standard, it may be safe to assume that in the remaining four studies, ${ }^{28,33,37,42}$ in which this item was unclear, interpretation was blinded.

Table 2.3 Methodological quality assessment results

\begin{tabular}{|c|c|c|c|c|c|c|c|c|c|c|c|c|c|c|c|c|c|}
\hline Study (first author) & 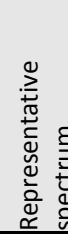 & 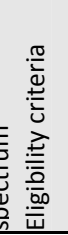 & 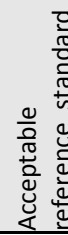 & 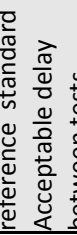 & 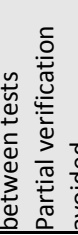 & 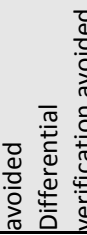 & 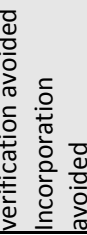 & 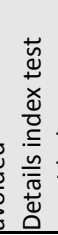 & $\frac{0}{\frac{0}{0}}$ & 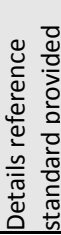 & 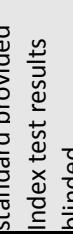 & 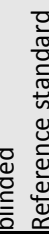 & 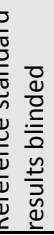 & 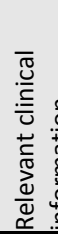 & 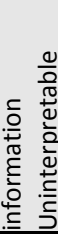 & 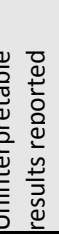 & 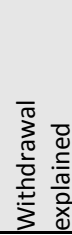 \\
\hline Al-Shawi $^{26}$ & $\mathrm{Y}$ & $\mathrm{N}$ & $\mathrm{Y}$ & $?$ & $\bar{Y}$ & $\mathrm{~N}$ & $\bar{Y}$ & $Y$ & $\bar{Y}$ & $\bar{Y}$ & $\bar{Y}$ & $\mathrm{Y}$ & & $?$ & $?$ & & $\mathrm{Y}$ \\
\hline Chang $^{27}$ & ? & $\mathrm{N}$ & Y & Y & $\mathrm{N}$ & $Y$ & Y & $Y$ & $Y$ & Y & Y & $Y$ & & ? & $\mathrm{N}$ & & $\mathrm{N}$ \\
\hline Cullen ${ }^{28}$ & $Y$ & $\mathrm{~N}$ & Y & Y & $\mathrm{N}$ & $Y$ & Y & $Y$ & & N & $?$ & $\mathrm{~N}$ & & ? & $?$ & & Y \\
\hline Farin $^{29}$ & ? & ? & Y & ? & $?$ & $\mathrm{~N}$ & $Y$ & $Y$ & & N & Y & $?$ & & ? & $\mathrm{N}$ & & $\mathrm{N}$ \\
\hline Frei $^{30}$ & $Y$ & $\mathrm{~N}$ & Y & ? & Y & $Y$ & Y & $Y$ & $Y$ & Y & Y & $?$ & & $Y$ & $\mathrm{~N}$ & & $\mathrm{~N}$ \\
\hline Iannotti ${ }^{31}$ & $Y$ & Y & Y & $?$ & Y & $\mathrm{Y}$ & Y & $Y$ & $Y$ & Y & Y & $\mathrm{N}$ & & $\mathrm{N}$ & $?$ & & Y \\
\hline Kayser $^{32}$ & ? & $\mathrm{N}$ & Y & ? & Y & $\mathrm{Y}$ & Y & $Y$ & $Y$ & Y & Y & $Y$ & & Y & $\mathrm{N}$ & & $\mathrm{N}$ \\
\hline Martin-Hervas ${ }^{33}$ & $Y$ & ? & Y & ? & Y & $Y$ & Y & $Y$ & & N & ? & $?$ & & ? & $\mathrm{N}$ & & $\mathrm{N}$ \\
\hline Milosavljevic ${ }^{34}$ & $Y$ & Y & Y & $\mathrm{N}$ & Y & $\mathrm{Y}$ & Y & $Y$ & & Y & Y & $?$ & & $?$ & $\mathrm{~N}$ & & $\mathrm{~N}$ \\
\hline Moosmayer ${ }^{35}$ & $Y$ & $\mathrm{~N}$ & Y & Y & Y & Y & $Y$ & Y & $Y$ & $\gamma$ & Y & $Y$ & & $\mathrm{~N}$ & $?$ & & Y \\
\hline Naredo $^{36}$ & $Y$ & Y & Y & Y & Y & $Y$ & Y & $Y$ & $Y$ & $\gamma$ & Y & $Y$ & & ? & $\mathrm{N}$ & & $\mathrm{N}$ \\
\hline Paavolainen ${ }^{37}$ & $Y$ & Y & Y & ? & $\mathrm{N}$ & $\mathrm{Y}$ & Y & $Y$ & $Y$ & $\gamma$ & ? & $?$ & & ? & $\mathrm{N}$ & & $\mathrm{N}$ \\
\hline $\operatorname{Read}^{38}$ & $Y$ & $\mathrm{~N}$ & Y & Y & Y & $Y$ & Y & $Y$ & $\mathrm{~N}$ & $N$ & Y & $\mathrm{N}$ & & ? & $\mathrm{N}$ & & $\mathrm{N}$ \\
\hline Roberts ${ }^{39}$ & $Y$ & $\mathrm{~N}$ & Y & ? & $?$ & $Y$ & Y & $Y$ & $Y$ & $\gamma$ & Y & $?$ & & ? & $?$ & & $?$ \\
\hline Rutten $^{40}$ & $Y$ & Y & Y & $Y$ & $\mathrm{~N}$ & $Y$ & Y & $Y$ & $Y$ & $\gamma$ & Y & $?$ & & ? & $\mathrm{N}$ & & Y \\
\hline Sonnabend ${ }^{41}$ & ? & ? & Y & Y & $?$ & $Y$ & Y & $Y$ & $Y$ & $\gamma$ & Y & $?$ & & ? & $\mathrm{N}$ & & $\mathrm{N}$ \\
\hline Swen ${ }^{42}$ & $Y$ & Y & Y & ? & Y & $Y$ & Y & $Y$ & $Y$ & $\gamma$ & $?$ & $?$ & & $\mathrm{~N}$ & $\mathrm{~N}$ & & $\mathrm{~N}$ \\
\hline Swen $^{43}$ & $Y$ & Y & Y & Y & Y & $Y$ & Y & $Y$ & $Y$ & $\gamma$ & Y & $?$ & & $\mathrm{~N}$ & $\mathrm{~N}$ & & $\mathrm{~N}$ \\
\hline Takagishi $^{44}$ & $Y$ & $\mathrm{~N}$ & Y & $?$ & $?$ & $Y$ & Y & $Y$ & $Y$ & $\gamma$ & Y & $?$ & & $?$ & $?$ & & $\mathrm{~N}$ \\
\hline Teefey $^{45}$ & $Y$ & Y & Y & Y & ? & $Y$ & Y & $Y$ & $Y$ & $\gamma$ & Y & $?$ & & ? & $?$ & & $\mathrm{~N}$ \\
\hline Teefey ${ }^{46}$ & $Y$ & Y & Y & $Y$ & $\mathrm{~N}$ & $Y$ & Y & $Y$ & $\mathrm{~N}$ & $N$ & Y & $\mathrm{N}$ & & ? & $?$ & & Y \\
\hline van Holsbeeck $^{47}$ & $Y$ & $\mathrm{~N}$ & Y & $\mathrm{N}$ & Y & $\mathrm{Y}$ & Y & $\mathrm{Y}$ & Y & $\gamma$ & Y & $?$ & & ? & $?$ & & $\mathrm{~N}$ \\
\hline Wiener ${ }^{48}$ & $Y$ & $\mathrm{~N}$ & $\mathrm{Y}$ & $?$ & $?$ & $\mathrm{~N}$ & $Y$ & $Y$ & Y & $\gamma$ & Y & $\mathrm{N}$ & & $?$ & $?$ & & $\mathrm{~N}$ \\
\hline Responses to quali & assess & smen & criteria & ia: $Y, y e$ & es; $N, \mathrm{r}$ & no; ?, u & unclear & & & & & & & & & & \\
\hline
\end{tabular}




\section{Data analysis}

Figures 2.2 and 2.3 show the forest plots for all target conditions and the HSROC curves for full- and partial-thickness tears, respectively. Pooled results for full- and partialthickness tears are presented in Table 2.4.

The 22 studies addressing full-thickness tears showed an overall pooled sensitivity of US of 0.95 (95\% Cl 0.90 to 0.97 ), specificity of 0.96 (95\% Cl 0.93 to 0.98 ), LR+ of 24.20 (95\% Cl 12.85 to 45.63 ) and LR- of 0.05 (95\% Cl 0.03 to 0.11 ). Studies using transducer frequencies $\geq 10 \mathrm{MHz}$ showed a pooled sensitivity of $0.98(95 \% \mathrm{Cl} 0.93-1.00)$ and a specificity of 0.94 ( $95 \% \mathrm{Cl} 0.87-0.97$ ). Pooled sensitivity and specificity remained above 0.90 in studies with a population average age $\leq 50$ years, a prevalence $<40 \%$, or without at least one key bias. None of the analyses found significant heterogeneity between the studies.

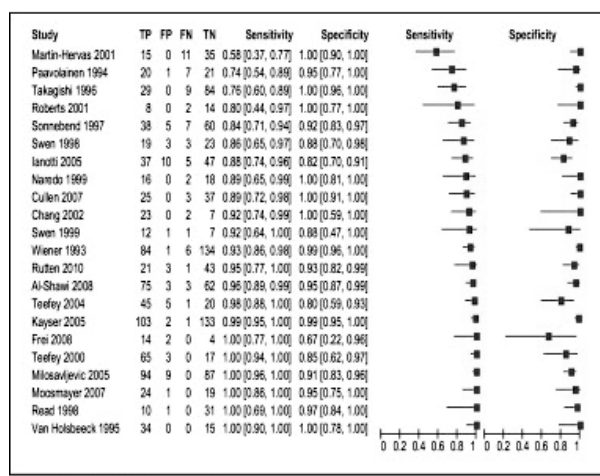

Full-thickness tear

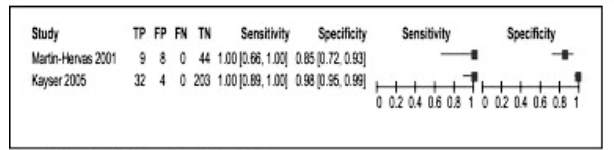

Calcifying tendonitis

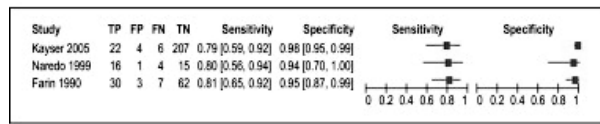

Subacromial bursitis

Figure 2.2 Forest plots per target condition

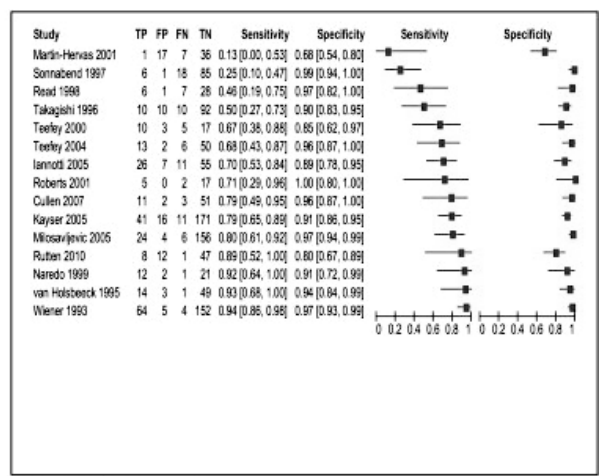

Partial-thickness tear

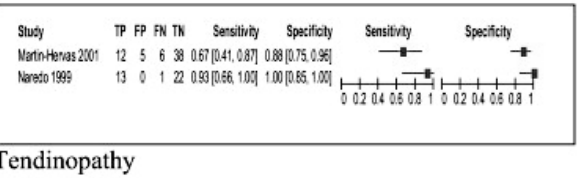




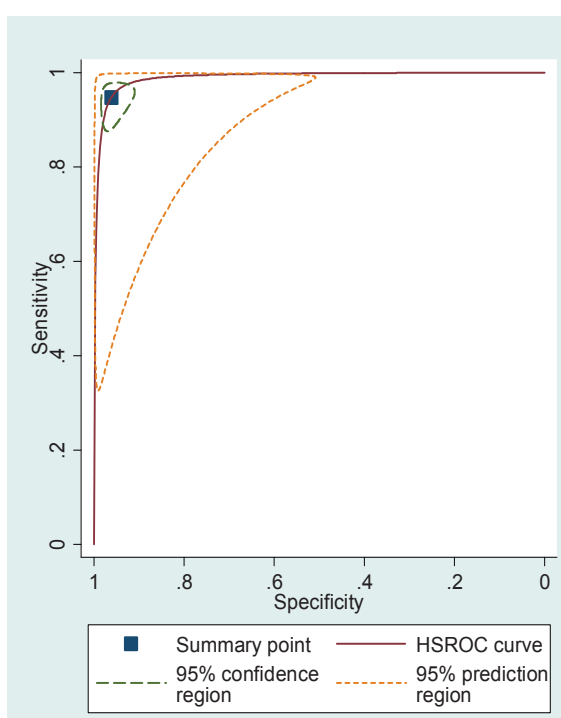

Full-thickness tears

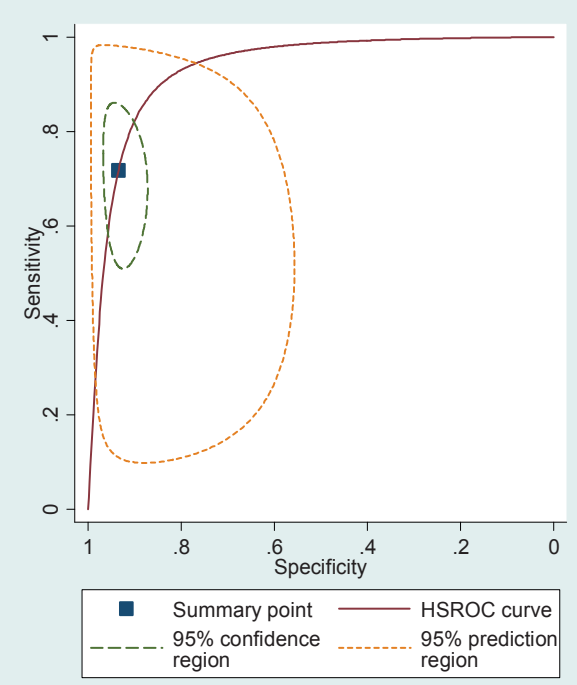

Partial-thickness tears

Figure 2.3 HSROC curves for full- and partial-thickness tears

The 15 studies addressing partial-thickness tears yielded a pooled sensitivity of 0.72 ( $95 \% \mathrm{Cl} 0.58$ to 0.83 ), specificity of 0.93 (95\% Cl 0.89 to 0.96 ), LR+ 10.91 (95\% Cl 6.26 to 19.02 ) and LR- 0.30 (95\% Cl 0.19 to 0.48 ). Subgroup analysis showed that sensitivity and specificity were higher in studies using transducer frequencies $\geq 10 \mathrm{MHz}$; sensitivity of 0.74 (95\% Cl $0.60-0.85)$ and a specificity of 0.97 (95\% Cl $0.94-0.98)$. Sensitivity decreased to 0.64 in studies with an average age $\leq 50$ years and 0.60 in those with a prevalence $<20 \%$. However, the overlap in $95 \% \mathrm{Cl}$ demonstrate that these differences are not statistically significant. None of the analyses found significant heterogeneity between the studies.

Due to statistical software limitations ( $\geq 4$ studies were needed) it was impossible to pool data and draw HSROC curves with point estimates for subacromial bursitis, tendinopathy or calcifying tendonitis. The accuracy statistics for the individual studies are presented in Table 2.5. For subacromial bursitis, sensitivity ranged from 0.79 to 0.81 , specificity from 0.94 to 0.98 , LR+ from 12.8 to 41.45 and $L R$ - from 0.20 to 0.22 . For tendinopathy, sensitivity ranged from 0.67 to 0.93 , specificity from 0.88 to 1.00 , LR+ from 5.73 to 41.47 and LR- from 0.07 to 0.38 . Sensitivity for calcifying tendonitis was 1.00 in both studies, with specificity ranging from 0.85 to $0.98, L R+$ from 6.50 to 51.75 and LR- from 0.02 to 0.06 . 


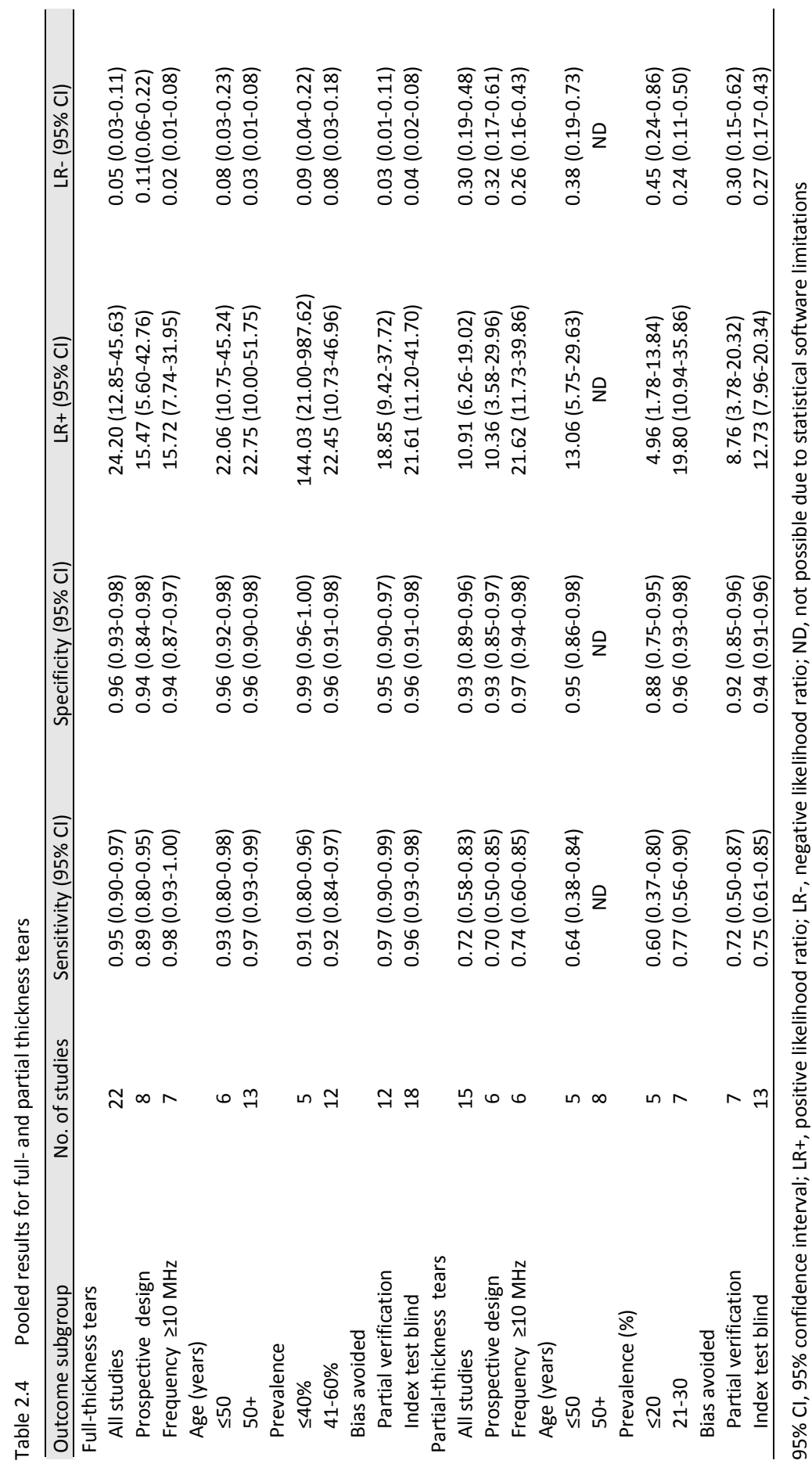




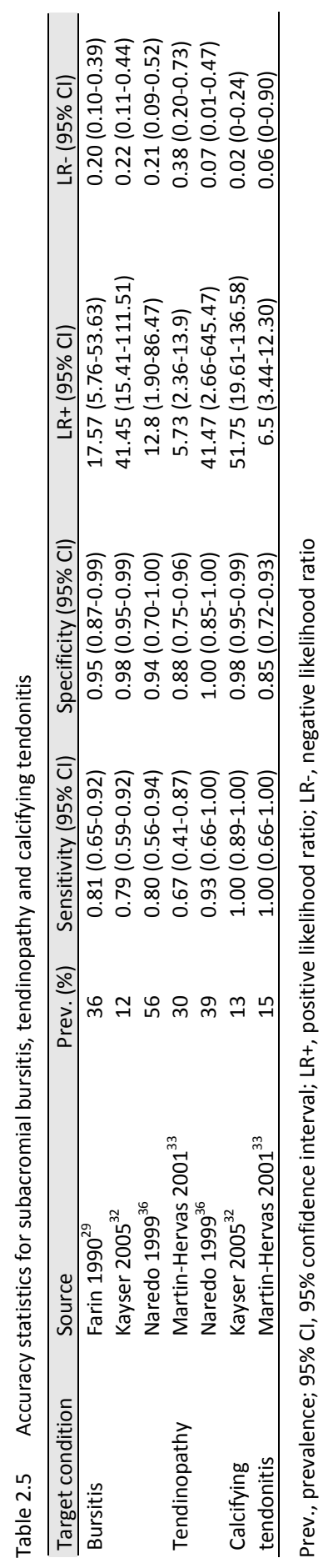




\section{Discussion}

\section{Statement of main results}

To our knowledge, this is the first time a systematic review has examined the accuracy of US to diagnose the full spectrum of subacromial disorders. Our comprehensive review identified data for tendinopathy, calcifying tendonitis, subacromial bursitis and partial- and full-thickness tears. The results indicate that in a secondary care setting in patients in whom conservative treatment has failed, US has a high diagnostic accuracy for most of these disorders.

Tests with high sensitivity and small LR- are most useful for ruling out disease, i.e., a negative result indicates that disease is not likely to be present. Tests with high specificity and high LR+ are most useful for ruling in disease, i.e., a positive test indicates that disease is likely to be present. ${ }^{49}$ Likelihood ratios summarize how many times more (or less) likely patients with the disease are to have a particular test result than patients without the disease. An LR+ above 10 and a LR- below 0.1 are considered to provide strong evidence to rule a diagnosis in or out, respectively. ${ }^{50}$

Overall, US has excellent diagnostic accuracy for diagnosing full-thickness tears, with a narrow $95 \% \mathrm{Cl}$. The results demonstrate that a positive US finding of a full-thickness tear provides convincing evidence that such a tear is in fact present, as it increases the odds of a full-thickness tear being present 24-fold ( $L R+24.20)$, well above the arbitrary threshold of $10 .^{50}$ In addition, a negative result rules out a full-thickness tear, as it decreases the odds 0.05 -fold (LR- 0.05 ), well below the 0.1 value commonly used for exclusion.

For partial-thickness tears, the individual studies show a wide variation in sensitivity, and a rather small variation in specificity. Four studies ${ }^{33,38,41,44}$ reported a low sensitivity $(<0.60)$, which might be explained by a small sample size, the use of low frequency transducers, disease progression and/or verification bias. It is easier to diagnose patients with partial-thickness tears (specificity 0.93, LR+ 10.91) than to rule it out (sensitivity 0.72 , LR- 0.30 ). Safely ruling out a partial-thickness tear in patients $\leq 50$ years and in populations with a prevalence $<20 \%$ is difficult (sensitivity 0.64 and 0.60 , respectively).

All studies were conducted in secondary care settings, and included patients with suspected rotator cuff disorders who had failed conservative treatment. In addition, 22 of the included 23studies evaluated full-thickness tears with surgery as the reference standard. This implies that these studies may have included a high proportion of more severe cases. Despite the high prevalence of subacromial disorders in primary care 
settings, we are uncertain whether the diagnostic value of US will be similar when used in primary care settings.

The small number of studies investigating subacromial bursitis, calcifying tendonitis and tendinopathy made statistical pooling impossible, so these results should be interpreted with caution. US has a high diagnostic accuracy for calcifying tendonitis. Although subacromial bursitis can be ruled in and out safely, and US appears to be able to accurately rule in tendinopathy, the sensitivity results of the two available studies conflict. $^{33,36}$ The low sensitivity in one study (0.67) might be explained by a rather small population, an outdated transducer frequency $(7.5 \mathrm{MHz})$ and possible disease progression bias. ${ }^{33}$

\section{Comparison with existing literature}

The two available systematic reviews on this topic were restricted to evaluating the accuracy of US for rotator cuff tears, ${ }^{17,21}$ whereas we also included results for tendinopathy, subacromial bursitis and calcifying tendonitis. Dinnes et al. ${ }^{17}$ included 38 studies, most of which had been carried out several years ago on equipment that has been superseded with higher frequency transducers, and were therefore not included in our review. On the other hand, we updated this review by adding 10 further studies. Compared to their results the overall sensitivity for full- and partial-thickness tears increased from 0.87 to 0.95 and from 0.67 to 0.72 , respectively, with specificity remaining unchanged. In comparison with de Jesus et al., ${ }^{21}$ we found 11 additional studies. This can be explained by the fact that we also included studies in which US had been performed by non-radiologists. They found an overall sensitivity for full- and partial thickness tears of 0.92 and 0.67 , respectively, with similar specificity compared to our results.

\section{Implications for clinical practice}

Our results suggest that US can have a positive impact on establishing a more accurate diagnosis in patients with shoulder complaints seen in a secondary care setting. For patients seen in primary care settings, the diagnostic value of US remains unclear. The combination of medical history, physical examination and US fulfil the need for diagnostic certainty and enables physicians to tailor treatment in most cases. For example, US is able to rule in and rule out full-thickness tears and hence allows rapid identification of those eligible to cuff repair surgery. There is evidence that cuff repair is effective. ${ }^{14,15,51}$ An example is given in the textbox below. 
Textbox

For an orthopaedic surgeon with 200 new patient encounters concerning shoulder pain per year, a prior probability of having a full-thickness tear of $40 \%$, using US (sensitivity of 0.91 and specificity of 0.99 ; see Table 2.4 ) this means that 73 true positive tears will be diagnosed, 7 false negative, 1 false positive, and 119 true negatives. The diagnosis will be incorrect for 8 patients; 7 cases with a possible surgery delay and 1 unnecessary surgical intervention. The use of MRI will not lead to a better result and will increase costs.

Ruling out partial-thickness tears seems to be the only major restriction in establishing an accurate diagnosis, especially in patients $<50$ years and in those with a low pre-test probability. Since diagnostic criteria for partial- and full-thickness tears overlap, and those for full-thickness tears are better defined, it is likely that ruling out is more difficult than ruling in. This implies that a positive test can in fact indicate a fullthickness tear instead of a partial-thickness one. Errors of diagnosis often consist of an inability to distinguish between small tears, ${ }^{26,31}$ but do not affect the planned approach. With some exceptions, e.g. active athletes, it can be questioned whether missing a small partial-thickness tear is harmful, as initial conservative treatment is recommended in these patients.

$M R I$ and MR arthrography can also be used to evaluate the painful shoulder. Our results confirm the evidence that US offers at least similar performance to MRI in diagnosing full-thickness tears (pooled sensitivity and specificity of MRI being 0.92 and 0.93 , respectively), ${ }^{21}$ and may be better at diagnosing partial-thickness tears ( 0.64 and 0.92). Evidence has shown that US is the more cost-effective test in secondary care to diagnose full- and partial thickness tears. ${ }^{17,21}$ This implies that US can be the method of choice in evaluating patients with shoulder pain, and MRI can be reserved for diagnosing concomitant abnormalities in those identified for surgery, or for those patients with suspected intra-articular disorders, e.g. labrum lesions. ${ }^{52} \mathrm{MR}$ arthrography is more accurate in ruling out a partial-thickness tear (pooled sensitivity of 0.86$).{ }^{21}$ This implies that MR arthrography can be performed in cases in which US is not definitive.

Rotator cuff tears are also common in asymptomatic and unselected populations. ${ }^{53-55}$ Since a certain percentage of the pathology found is not responsible for the reported complaints, physicians should realize that there might not be a straightforward correlation between US findings and symptoms. To prevent unnecessary treatment of 
asymptomatic US pathology, US findings have to be linked to medical history and physical examination.

Remarkably, only two studies have reported on tendinopathy. ${ }^{33,36}$ Tendinopathy refers to a degenerative tendon, ${ }^{56-58}$ and overuse in tendinopathy can result in loss of tendon structure, and a decrease in biomechanical properties of the tendon. This might eventually result in tendon tearing and chronic subacromial bursal reactions. In our experience, tendinopathy is present in many patients with shoulder complaints, especially in those with tears. This should be kept in mind during rehabilitation.

\section{Strengths and limitations of the study}

We carried out an extensive literature search using the methods recommended in the Cochrane Handbook on Systematic Reviews of Diagnostic Test, and current statistical methods. Two reviewers independently screened the titles and abstracts, assessed the quality of the studies and extracted data. This is the first systematic review providing accuracy results for all subacromial disorders.

We updated the reference list of the first published systematic review on this topic, with a comparable search strategy, while realizing that their search was limited to articles in English. ${ }^{17}$ Contacting authors did not increase the number of studies included. Although this may have resulted in a potential loss of relevant data, a substantial number of studies were included, and no statistical heterogeneity was detected. The studies also appear to be clinically homogeneous: all studies were conducted in secondary care settings, and included patients with suspected rotator cuff disorders who had failed conservative treatment. This strengthens our results.

Unfortunately, incomplete reporting hampers the evaluation of potential sources of bias in diagnostic accuracy studies, although it is not entirely clear how individual quality items may affect estimates of diagnostic accuracy. ${ }^{59,60}$ Only seven studies reported on the duration of symptoms, and it was not possible to compare between acute and chronic complaints. Partial verification bias was only clearly avoided in half of the studies, and disease progression bias might have influenced the results. However, these design features hardly seem to affect estimates of diagnostic accuracy. ${ }^{59}$ As recommended, we did not incorporate a combined quality score in our review, but investigated the association of individual quality items with estimates of diagnostic accuracy. ${ }^{61}$ We found that two individual quality items (partial verification avoided and index test blind), identified beforehand as key biases, did not significantly influence the estimates. This can be due to a true lack of influence or due to a lack of power to detect influence. 
The results of this review are presented graphically in order to provide both a detailed overview of the results (diagnostic accuracy) and to communicate information that can be used to inform clinicians (predictive value). ${ }^{62}$ Unfortunately, limitations of the statistical software we used made it impossible to pool data and draw HSROC curves with point estimates for all target conditions.

\section{Unanswered questions and future research}

Not a single study was conducted in a primary care setting, nor have acute complaints been a specific subject of study. This is not entirely surprising, considering US is rarely performed in primary care settings, and US is more or less reserved for patients with chronic complaints. The prognosis of shoulder complaints is poor, and one might say that patients with shoulder complaints are predetermined to develop a chronic condition if they do not receive adequate treatment. This might lead to significantly reduced health and high costs. Management should focus on prevention of progression and early intervention in these complaints. This seems to require collecting more information, at an earlier stage, about the quality and dynamics of the rotator cuff. Future research should focus on diagnostic accuracy studies in settings with lower prevalences, follow-up studies of patients with shoulder complaints in primary and secondary care settings to evaluate the natural history and epidemiology, and studies into the cost-effectiveness of management that includes diagnostic US in treatment decisions.

\section{Conclusions}

In order to achieve appropriate treatment decisions, we strongly recommend the use of US in patients in whom conservative treatment has failed, to rule full-thickness tears in or out, to rule in partial-thickness tear, and to a lesser extent to diagnose tendinopathy, subacromial bursitis and calcifying tendonitis. The diagnostic accuracy of US in patients with acute complaints remains to be determined. 


\section{References}

1. Feleus A, Bierma-Zeinstra SM, Miedema HS, Bernsen RM, Verhaar JA, Koes BW. Incidence of nontraumatic complaints of arm, neck and shoulder in general practice. Man Ther. 2008;13:426-433.

2. Picavet HS, Schouten JS. Musculoskeletal pain in the Netherlands: prevalences, consequences and risk groups, the DMC(3)-study. Pain. 2003;102:167-178.

3. Bot SD, van der Waal JM, Terwee CB, et al. Incidence and prevalence of complaints of the neck and upper extremity in general practice. Ann Rheum Dis. 2005;64:118-123.

4. Lewis J, Tennent D, eds. How effective are diagnostic tests for the assessment of rotator cuff disease of the shoulder? London: Blackwell, BMJ Books; 2007. MacAuley D, Best TM, eds. Evidence based sports medicine.

5. Bigliani LU, Levine WN. Subacromial impingement syndrome. J Bone Joint Surg Am. 1997;79:1854-1868.

6. Neer CSn. Impingment lesions. Clin Orthop rel Res. 1983;173:70-77.

7. Fischer AF, Dexter WW, eds. How evidence-based is our examination of the shoulder. London: Blackwell, BMJ Books; 2007. MacAuly D, Best TM, eds. Evidence based sports medicine.

8. Hughes PC, Taylor NF, Green RA. Most clinical tests cannot accurately diagnose rotator cuff pathology: a systematic review. Aust J Physiother. 2008;54:159-170.

9. Bamji AN, Erhardt CC, Price TR, Williams PL. The painful shoulder: can consultants agree? $\mathrm{Br} J$ Rheumatol. 1996;35:1172-1174.

10. de Winter AF, Jans MP, Scholten RJ, Deville W, van Schaardenburg D, Bouter LM. Diagnostic classification of shoulder disorders: interobserver agreement and determinants of disagreement. Ann Rheum Dis. 1999;58:272-277.

11. Liesdek C, van der Windt DAWM, Koes BW, Bouter LM. Soft-tissue disorders of the shoulder. A study of inter-observer agreement between general practitioners and physiotherapists and an overview of physiotherapeutic treatment. Physiotherapy. 1997;83:12-17.

12. Baring T, Emery RJH, Reilly P. Management of rotator cuff disease: specific treatment for specific disorders. Best Pract Res Clin Rheumatol. 2007;21:279-294.

13. Kuhn JE. Exercise in the treatment of rotator cuff impingement: a systematic review and a synthesized evidence-based rehabilitation protocol. J Shoulder Elbow Surg. 2009;18:138-160.

14. Millett PJ, Wilcox RB, 3rd, O'Holleran JD, Warner JJ. Rehabilitation of the rotator cuff: an evaluationbased approach. J Am Acad Orthop Surg. 2006;14:599-609.

15. Lahteenmaki HE, Hiltunen A, Virolainen $P$, Nelimarkka O. Repair of full-thickness rotator cuff tears is recommended regardless of tear size and age: a retrospective study of 218 patients. J Shoulder Elbow Surg. 2007;16:586-590.

16. Allen GM, Wilson DJ. Ultrasound of the shoulder. Eur J Ultrasound. 2001;14:3-9.

17. Dinnes J, Loveman E, Mclntyre L, Waugh N. The effectiveness of diagnostic tests for the assessment of shoulder pain due to soft tissue disorders: a systematic review. Health Technol Assess. 2003;7:1-166.

18. Jacobson JA, van Holsbeeck MT. Musculoskeletal ultrasonography. Orthop Clin North Am. 1998;29: 135-167.

19. Middleton W, Teefey S, Yamaguchi K. Sonography of the rotator cuff: analysis of interobserver variability. Am J Roentgenol. 2004;183:1465-1468.

20. Martinoli C, Bianchi S, Prato N, et al. US of the shoulder: non-rotator cuff disorders. Radiographics. 2003;23:381-401.

21. de Jesus JO, Parker L, Frangos AJ, Nazarian LN. Accuracy of MRI, MR arthrography, and ultrasound in the diagnosis of rotator cuff tears: a meta-analysis. Am J Roentgenol. 2009;192:1701-1707.

22. Handbook for DTA reviews. Available at: http://srdta.cochrane.org/en/authors.html

23. Whiting P, Rutjes AW, Dinnes J, Reitsma J, Bossuyt PM, Kleijnen J. Development and validation of methods for assessing the quality of diagnostic accuracy studies. Health Technol Assess. 2004;8:iii, 1-234.

24. Harbord RM, Whiting P, Sterne JA, et al. An empirical comparison of methods for meta-analysis of diagnostic accuracy showed hierarchical models are necessary. J Clin Epidemiol. 2008;61:1095-1103. 
25. Reitsma JB, Glas AS, Rutjes AW, Scholten RJ, Bossuyt PM, Zwinderman AH. Bivariate analysis of sensitivity and specificity produces informative summary measures in diagnostic reviews. J Clin Epidemiol. 2005;58:982-990.

26. Al-Shawi A, Badge R, Bunker T. The detection of full thickness rotator cuff tears using ultrasound. J Bone Joint Surg. 2008;90:889-892.

27. Chang CY, Wang SF, Chiou HJ, Ma HL, Sun YC, Wu HD. Comparison of shoulder ultrasound and MR imaging in diagnosing full-thickness rotator cuff tears. Clin Imaging. 2002;26:50-54.

28. Cullen DM, Breidahl WH, Janes GC. Diagnostic accuracy of shoulder ultrasound performed by a single operator. Australas Radiol. 2007;51:226-229.

29. Farin PU, Jaroma H, Harju A, Soimakallio S. Shoulder impingement syndrome: sonographic evaluation. Radiology. 1990;176:845-849.

30. Frei R, Chladek P, Trc T, Kopecny Z, Kautzner J. Arthroscopic evaluation of ultrasonography and magnetic resonance imaging for diagnosis of rotator cuff tear. Ortop Traumatol Rehabil. 2008;10: 111-114.

31. lannotti JP, Ciccone J, Buss DD, et al. Accuracy of office-based ultrasonography of the shoulder for the diagnosis of rotator cuff tears. J Bone Joint Surg Am. 2005;87:1305-1311.

32. Kayser R, Hampf S, Pankow M, Seeber E, Heyde CE. Validity of ultrasound examinations of disorders of the shoulder joint. Ultraschall Med. 2005;26:291-298.

33. Martin-Hervas C, Romero J, Navas-Acien A, Reboiras JJ, Munuera L. Ultrasonographic and magnetic resonance images of rotator cuff lesions compared with arthroscopy or open surgery findings. J Shoulder Elbow Surg. 2001;10:410-415.

34. Milosavljevic J, Elvin A, Rahme H. Ultrasonography of the rotator cuff: a comparison with arthroscopy in one-hundred-and-ninety consecutive cases. Acta Radiol. 2005;46:858-865.

35. Moosmayer S, Heir S, Smith HJ. Sonography of the rotator cuff in painful shoulders performed without knowledge of clinical information: results from 58 sonographic examinations with surgical correlation. $J$ Clin Ultrasound. 2007;35:20-26.

36. Naredo $A E$, Aguado $P$, Padron $M$, et al. A comparative study of ultrasonography with magnetic resonance imaging in patients with painful shoulder. J Clin Rheumatol. 1999;5:184-192.

37. Paavolainen $\mathrm{P}$, Ahovuo J. Ultrasonography and arthrography in the diagnosis of tears of the rotator cuff. J Bone Joint Surg Am. 1994;76:335-340.

38. Read JW, Perko M. Shoulder ultrasound: diagnostic accuracy for impingement syndrome, rotator cuff tear, and biceps tendon pathology. J Shoulder Elbow Surg. 1998;7:264-271.

39. Roberts CS, Walker JA, 2nd, Seligson D. Diagnostic capabilities of shoulder ultrasonography in the detection of complete and partial rotator cuff tears. Am J Orthop. 2001;30:159-162.

40. Rutten MJ, Spaargaren GJ, van Loon T, de Waal Malefijt MC, Kiemeney LA, Jager GJ. Detection of rotator cuff tears: the value of MRI following ultrasound. Eur Radiol. 2010;20:450-457.

41. Sonnabend DH, Hughes JS, Giuffre BM, Farrell R. The clinical role of shoulder ultrasound. Aust N Z J Surg. 1997;67:630-633.

42. Swen WA, Jacobs JW, Algra PR, et al. Sonography and magnetic resonance imaging equivalent for the assessment of full-thickness rotator cuff tears. Arthritis Rheum. 1999;42:2231-2238.

43. Swen WA, Jacobs JW, Neve WC, Bal D, Bijlsma JW. Is sonography performed by the rheumatologist as useful as arthrography executed by the radiologist for the assessment of full thickness rotator cuff tears? J Rheumatol. 1998;25:1800-1806.

44. Takagishi K, Makino K, Takahira N, Ikeda T, Tsuruno K, Itoman M. Ultrasonography for diagnosis of rotator cuff tear. Skeletal Radiol. 1996;25:221-224.

45. Teefey SA, Hasan SA, Middleton WD, Patel M, Wright RW, Yamaguchi K. Ultrasonography of the rotator cuff. A comparison of ultrasonographic and arthroscopic findings in one hundred consecutive cases. J Bone Joint Surg Am. 2000;82:498-504.

46. Teefey SA, Rubin DA, Middleton WD, Hildebolt CF, Leibold RA, Yamaguchi K. Detection and quantification of rotator cuff tears. Comparison of ultrasonographic, magnetic resonance imaging, and arthroscopic findings in seventy-one consecutive cases. J Bone Joint Surgery Am. 2004;86-A:708-716.

47. van Holsbeeck MT, Kolowich PA, Eyler WR, et al. US depiction of partial-thickness tear of the rotator cuff. Radiology. 1995;197:443-446. 
48. Wiener SN, Seitz WH, Jr. Sonography of the shoulder in patients with tears of the rotator cuff: accuracy and value for selecting surgical options. Am J Roentgenol. 1993;160:103-107; discussion 109-110.

49. Altman DG, Bland JM. Diagnostic tests. 1: Sensitivity and specificity. BMJ. 1994;308:1552.

50. Deeks JJ, Altman DG. Diagnostic tests 4: likelihood ratios. BMJ. 2004;329:168-169.

51. Burkhart SS, Barth JR, Richards DP, Zlatkin MB, Larsen M. Arthroscopic repair of massive rotator cuff tears with stage 3 and 4 fatty degeneration. Arthroscopy. 2007;23:347-354.

52. Matava MJ, Purcell DB, Rudzki JR. Partial-thickness rotator cuff tears. Am J Sports Med. 2005;33: 1405-1417.

53. Milgrom C, Schaffler M, Gilbert S, van Holsbeeck M. Rotator-cuff changes in asymptomatic adults. The effect of age, hand dominance and gender. J Bone Joint Surg. 1995;77:296-298.

54. Reilly P, Macleod I, Macfarlane R, Windley J, Emery RJH. Dead men and radiologists don't lie: a review of cadaveric and radiological studies of rotator cuff tear prevalence. Ann R Coll Surg Engl. 2006;88: 116-121.

55. Sher JS, Uribe JW, Posada A, Murphy BJ, Zlatkin MB. Abnormal findings on magnetic resonance images of asymptomatic shoulders. J Bone Joint Surg Am. 1995;77:10-15.

56. Khan KM, Cook JL, Bonar F, Harcourt P, Astrom M. Histopathology of common tendinopathies. Update and implications for clinical management. Sports Med. 1999;27:393-408.

57. Puddu G, Ippolito E, Postacchini F. A classification of Achilles tendon disease. Am J Sports Med. 1976;4: 145-150.

58. Perugia L, Ippolitio E, Postacchini F. A new approach to the pathology, clinical features and treatment of stress tendinopathy of the Achilles tendon. Ital J Orthop Traumatol. 1976;2:5-21.

59. Rutjes AW, Reitsma JB, Di Nisio M, Smidt N, van Rijn JC, Bossuyt PM. Evidence of bias and variation in diagnostic accuracy studies. CMAJ. 2006;174:469-476.

60. Leeflang $M$, Reitsma J, Scholten R, et al. Impact of adjustment for quality on results of metaanalyses of diagnostic accuracy. Clin Chem. 2007;53:164-172.

61. Whiting $P$, Harbord R, Kleijnen J. No role for quality scores in systematic reviews of diagnostic accuracy studies. BMC Med Res Methodol. 2005;5:19.

62. Whiting PF, Sterne JA, Westwood ME, et al. Graphical presentation of diagnostic information. BMC Med Res Methodol. 2008;8:20. 


\section{Chapter}

GPs' perspectives on the diagnostic work-up in patients with shoulder pain: a qualitative study

Ramon PG Ottenheijm, Nicolle JJM Hesselmans, Anouk Kemper, Albine Moser, Rob A de Bie, Geert-Jan Dinant, Jochen WL Cals J Clin Eval Pract 2014;20:239-45 


\section{Abstract}

\section{Rationale, aims and objectives}

The diagnostic work-up of patients with shoulder pain in general practice is complex. GP guidelines advise a pragmatic diagnostic work-up in which additional imaging has a limited role. However, diagnostic ultrasounds are increasingly ordered by GPs, which seems to reflect complexity in management of shoulder pain. This study aimed to explore GPs' perspectives on the diagnostic work-up of patients with shoulder pain.

\section{Method}

This study has a qualitative exploratory design with an inductive approach and was carried out in Dutch general practice. The study population consisted of 18 Dutch GPs who were sampled purposefully with a spread in clinical experience and ordering diagnostic ultrasound. Data were gathered by means of semistructured interviews and analysed following principles of the constant comparative method.

\section{Results}

Three main categories with subcategories emerged that captured the diagnostic work-up of shoulder pain: variety in diagnostic classifications ((non-)specific diagnosis, and interdisciplinary differences), establishing strategies for diagnostic work-up (use of existing tools, and motives to deviate from existing tools), and strategies dealing with diagnostic uncertainties (accepting diagnostic uncertainties, diagnostic imaging tests, and interdisciplinary consultation and referral).

\section{Conclusions}

Despite the availability of evidence-based shoulder guidelines, GPs experience uncertainties during diagnostic work-up, and apply different strategies when dealing with these uncertainties. At some point, GPs as well as patients seem to have a need for a specific diagnosis. Currently, there appears to be little agreement if, or in which phase of shoulder pain, diagnostic ultrasound is useful or indicated. 


\section{Introduction}

Shoulder pain is the third most common musculoskeletal health problem managed in general practice, with up to 100 new patients per general practitioner (GP) consulting per year and a prevalence of $17-20 \%$ in the general population. ${ }^{1-3}$ Shoulder pain is a complex problem with an unfavourable outcome. About $40 \%$ of the patients still experience pain after one year, ${ }^{4}$ and $40-50 \%$ reconsult their GP. ${ }^{3,5,6}$ Therefore, improvement of the general practice management of shoulder pain has the potential to improve prognosis of shoulder pain.

Since 1999 the Dutch College of General Practitioners (DCGP) advises to refrain from using the diagnostic Cyriax classification, which was based on patho-anatomical characteristics, e.g. supraspinatus tendinitis or acute bursitis, to diagnose shoulder pain. ${ }^{3}$ This advice was based on research that illustrated the lack of interobserver agreement on the appropriate diagnostic criteria and various classifications used for

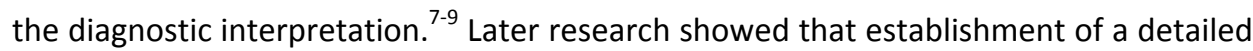
classification of shoulder pain is not possible. ${ }^{10}$ Therefore, the shoulder guidelines issued by the DCGP and the National Institute for Health and Clinical Excellence (available as Clinical Knowledge Summaries) in the UK, propose a non-specific classification based on pain and physical examination findings, which can be linked to the origin of the complaints (e.g. subacromial or a specific joint). ${ }^{3,11}$ Studies in The Netherlands and UK show that GPs in most cases record a non-specific diagnosis., ${ }^{5,6}$ In general practice, subacromial pathology, e.g. rotator cuff disorders, is responsible for the pain in $80 \%$ of the cases with shoulder pain. ${ }^{3}$ Shoulder guidelines advise that additional imaging tests, ultrasound (US) in particular, should be reserved for use in patients in whom conservative treatment fails and in red flag situations. ${ }^{3,11-13}$ However, research in general practice in Australia and the USA shows that in $40-80 \%$ of the patients with a new presentation of shoulder pain at least one imaging test is ordered, ${ }^{14-18}$ and that in The Netherlands and Australia there has been a rapid increase in the use of US. ${ }^{19,20}$ Also, GPs show an overreliance on early specialist referral. ${ }^{16}$

The diagnostic work-up in patients with shoulder pain in general practice still seems complicated, and limited research is available about GPs experiences and problems during this diagnostic work-up, and their motives for ordering US in these patients. Insight in these experiences and motives would be valuable, as it might provide a basis for clinical management improvement, GP education and input for further research. The aim of this study is to obtain a deeper understanding about GPs perspectives on the diagnostic work-up of patients with shoulder pain in general practice, with the following questions:

- Which diagnostic classifications do GPs use in patients with shoulder pain? 
- Which problems do GPs experience during the diagnostic work-up of patients with shoulder pain?

- How do GPs deal with problems during their diagnostic work-up?

\section{Methods}

In an exploratory qualitative design, we performed semi-structured interviews, as this is best suited to achieve a deeper understanding of experiences and motives from the perspective of the GP. ${ }^{21}$

\section{Participants}

We invited 48 GPs from the wider Maastricht region to participate. Purposive sampling was used to ensure a wide range in clinical experience (more or less than 10 years of experience) To obtain a wide range of perspectives on the diagnostic use of US, based on data provided by the local diagnostic centres, we additionally selected GPs that ordered more than 30 shoulder US per year, reflecting high-end users. ${ }^{22,23}$ After receiving full information of the study, participants gave informed consent. This research did not require approval of an ethics committee according to Dutch legislation. $^{24}$ In the Netherlands, all patients are enlisted with a GP; GPs operate as 'gatekeepers' to secondary care. Management of shoulder pain is primarily done by GPs, and GPs have direct access to imaging techniques, such as US.

\section{Data collection}

The face-to- face interviews took place between October 2012 and January 2013. To explore each research question, we designed a topic list with open ended questions (Box 1.1), and piloted this in two test-interviews. The questions were partly adapted, as new codes arose during analysis. ${ }^{25}$ The average interview time was 30 minutes. All interviews were audio-taped and transcribed verbatim.

\section{Box 1.1 Topics included in the semi-structured interviews}

- Diagnosing patients with shoulder pain

- Classification of patients with shoulder pain

- Barriers in the diagnostic work-up

- The role of imaging and US in particular 
Transcription and analyses were carried out by two researchers ( $\mathrm{NH}, \mathrm{AK})$. The responses were analysed inductively, using the constant comparative method. ${ }^{25}$ First, the interviews were read to get a general impression. Then text fragments were selected which provided an answer to the research questions. Next, open coding was used. ${ }^{23,25}$ Subsequently, we compared and contrasted these codes, and grouped them in larger (sub)categories. After analysis of 16 interviews, categorical saturation occurred. The remaining two interviews were used to confirm and verify the analysis. Coding of the data was supported by NVivo software.

To assure internal validity GPs were provided with the transcripts to comment on the accuracy (member checking). ${ }^{26}$ A third researcher (RO) supervised the analysis process (research triangulation). ${ }^{26}$ To obtain basic coding elements, the first three transcripts were analysed and coded separately by these three researchers, followed by analytical discussions and agreement on the coding. The third researcher randomly coded five of the additional fifteen interviews and was consulted if necessary. Throughout the inquiry, all authors held regular meetings to review and explore the analysis process (peer debriefing). ${ }^{26}$

\section{Results}

In total, 48 GPs were invited; 18 of these were interviewed, 17 did not reply, 11 declined to participate, and two were unable to participate within the study period. Characteristics of the participating GPs are presented in Table 3.1.

\begin{tabular}{lr} 
Table $3.1 \quad$ Characteristics of participating GPs \\
\hline & $\mathrm{n}$ \\
\hline Sex & 13 \\
$\quad$ Male & 5 \\
$\quad$ Female & \\
Experience, years & 8 \\
$\quad<10$ (less experienced) & 10 \\
>10 (experienced) & \\
Practice type & 2 \\
$\quad$ Single handed & 16 \\
$\quad$ Group & \\
Shoulder ultrasounds ordered per year & 14 \\
$\quad<30$ (low-end US user) & 4 \\
$\quad \geq 30$ (high-end US user) & \\
\hline
\end{tabular}


Three main categories emerged that captured the diagnostic work-up of shoulder pain: variety in diagnostic classifications, establishing strategies for diagnostic work-up, and strategies dealing with diagnostic uncertainties (Box 3.2).

Box 3.2 Categories and subcategories

1. Variety in diagnostic classifications
- Non-specific diagnosis
- Specific diagnosis
- Interdisciplinary differences
2. Establishing strategies for diagnostic work-up
- Use of existing tools
- Motives to deviate from existing tools
$\quad$ Experience
$\quad$ Barriers to guideline adherence
3. Strategies dealing with diagnostic uncertainties
- Accepting diagnostic uncertainties
- Diagnostic imaging tests
- Interdisciplinary consultation and referral

\section{Category 1: Variety in diagnostic classifications}

GPs use several diagnostic classifications in patients with shoulder pain. Initially, GPs classify shoulder pain in terms of a general, non-specific diagnosis or a specific, pathoanatomical diagnosis. Diversity in use exists within these two groups, and these diagnostic classifications were not always mutually exclusive. Consequently, this affects interdisciplinary agreement and cooperation.

\section{Non-specific diagnosis}

Most GPs accept that it is not possible to establish a specific patho-anatomical diagnosis. GPs who classified non-specific diagnoses, mostly distinguished between a glenohumeral or subacromial origin, based on limited signs and symptoms information. However, some of these GPs extend this classification with labelling the biceps or supraspinatus tendon, or AC-joint.

"At least, I have a method, I want to know if it is a capsular, a joint or a subacromial problem.

Only when I think I can be more specific by saying it is the supraspinatus, I will note that. Otherwise I will call it subacromial." (GP 16, experienced, high-end US user) 
In order to target treatment, others use classifications based on duration or etiology.

"Then you differentiate between a more chronic or an acute complaint. And this is an inflammation, inflammation-related disease, or more traumatic. For further treatment, it is important to differentiate" (GP 8, less experienced, low-end US user)

\section{Specific diagnosis}

Although GPs admit that a specific diagnosis is not always possible or necessary to target treatment, they prefer a patho-anatomical diagnosis, based on extensive examination. Cyriax's classification is mentioned as a basis by some GPs.

"In most cases I diagnose tendinitis or bursitis, or a joint problem. (....). So, I try to be as specific as possible. Also to differentiate between tendons, and to see if it's impingement. Although this isn't always possible by physical examination." (GP 11, less experienced, low-end US user)

Other reasons for a specific diagnostic approach were to decrease the need for diagnostic imaging by earning trust from the patients. One GP clearly describes great benefits in trust as a patient outcome.

"Yes, one advantage is that you extensively examine your patients. (....) Gain trust! And often a correct diagnosis, with subsequently less need for additional tests. Maybe these are ordered too much." (GP 18, experienced, high-end US user)

\section{Interdisciplinary differences}

Several GPs mention that the variety in diagnostic work-up, and subsequently different diagnostic classification lead to difficulties in communication and diagnostic disagreements between professionals. Moreover, patients get confused about their diagnosis.

"Orthopedic surgeons think quite differently in the diagnostic work-up compared to what is stated in the GP guideline? And you will have to communicate with them. A number of patients also see the physiotherapist. And I think it is quite hard to communicate in such vague terms with your colleagues you are regularly dealing with. So, as well in communication towards patients as to colleagues."(GP 5, experienced, low-end US user) 


\section{Category 2: Establishing strategies for diagnostic work-up}

All GPs have established their individual diagnostic work-up routines consisting of medical history, physical examination, ordering diagnostic tests, and referral. These routines are based on tools such as continuing medical education (CME) teaching, e.g. workshops, and reading the shoulder guideline.

\section{Use of existing tools}

GPs stress the importance of implementing evidence by CME, while this positively affects their efficiency in diagnostic work-up. For example, they had training by orthopedic surgeons.

"I once learned from an orthopedic surgeon to limit my physical examination. It makes no sense to do several tests. (...) The orthopedic surgeon said: "Listen, if you want to differentiate in the shoulder, you do not need that much tests. You don't need to examine all movements. Only a few tests are necessary." (GP 4, experienced, low-end US user)

For several GPs, the shoulder guideline provides a pragmatic and easy approach, which confirms their method to be sufficient. However, other GPs mentioned that their own experiences and preferences played a major role in the establishment of their diagnostic work-up.

\section{Motives to deviate from existing tools}

\section{Experience}

With increasing levels of clinical experience, evidence based guidelines seem to have less impact on diagnostic work-up. Some GPs experience effective diagnostic work-up by applying Cyriax's classification. Some expressed doubts about the diagnostic benefit of changing diagnostic work-ups and mention this as a motive to hold on to their approach.

"During vocational training I experienced the right approach, which sticks best. Each five years a different approach is advised, which make me think: Was it that bad the last five years? Well, I don't think so. So, compared to fifteen years ago, I noticed that those guidelines have less impact." (GP 16, experienced, high-end US user) 
In addition, several GPs explain their work-up as working with so called 'illness scripts', which develop over time: quick-scan, first hypothesis and testing this by most appropriate questions and tests.

"Very quickly, I have an idea of what it might be. Then I will check if that is correct. But previously, you were free from ideas, and then you did all tests according to the protocol." (GP 13, less experienced, low-end US user)

Barriers to guideline adherence

Some GPs specifically reported perceived barriers when adhering to the guideline. Some perceive that guideline recommendations were incomplete and superficial. GPs where critical in applying guidelines in general and more particular, perceive a lack of applicability, specifically to the individual patient.

"Nowadays, people need to know exactly what is going on. (...) This is also applicable to their health. (..) If I would stick to the guideline, patients would have to wait too long for US or an X-ray." (GP 15, experienced, high-end US user)

Previous practice and professional experiences seem to influence not applying the guideline. Some GPs prefer to stick to their habitual diagnostic work-up based on acquired knowledge.

"Some kind of knowledge network constructed in my head, which is really hard to change and switch to a different approach. Particular this applies in shoulder pain." (GP 5, experienced, low-end US user)

\section{Category 3: Strategies dealing with diagnostic uncertainties}

Despite established work-ups, GPs still report difficulties diagnosing shoulder pain by physical examination, as the shoulder is seen as a complex joint. Some GPs judge their diagnostic knowledge to be insufficient. Consequently, GPs have to deal with a certain amount of uncertainty.

\section{Accepting diagnostic uncertainties}

Some GPs mentioned that dealing with uncertainties is a typical characteristic of general practice, and tend to accept this uncertainty, as a high proportion of complaints are self-limiting. 
Factors influencing GPs' need around resolving uncertainties in the diagnostic workup were mostly patient dependent including: chronic complaints, therapy resistance, age, context, and patients' need for reassurance. Some apply a pragmatic approach. If the burden of shoulder pain for patients gets too high they request diagnostic imaging tests. Also, GPs mentioned so called "gut-feelings" (a sense of alarm), which are not related to specific complaints, but are rather intuitive.

"Well yes, maybe due to my gut-feelings, which you develop as GP. It's some kind of automatism. There are no specific complaints requiring an US imperatively." (GP 12, less experienced, low-end US user)

GPs expand their diagnostic work-up either by ordering additional imaging tests or in some cases consult or refer to physiotherapist or orthopedic surgeon.

\section{Diagnostic imaging tests}

In general, X-rays are ordered in patients with trauma or suspicion of osteoarthritis. However, there seems to be little agreement among GPs in which phase of shoulder pain US is indicated. GPs mainly agree that when a traumatic tendon tear is suspected, in the short term US is indicated. In the other non-chronic cases, several GPs do not mention advantages of US.

"Look, sometimes patients ask the question: is it not possible to order an US? (...) But, then I try to explain that when it concerns a first presentation of shoulder pain, US has no additional value. Of course, with exception of traumatic events, I try to explain that it is more useful to figure out what is going on by using treatment as a diagnostic tool." (GP 8, less experienced, low-end US user)

Subsequently, these GPS usually order US in case of therapy resistance or chronic complaints. In contrast, a few GPs highly disagree with this approach. As tailored treatment is possible in the non-chronic phase, they consider US only to be valuable in this non-chronic phase of shoulder pain.

"Tendon repair could have been the solution, at least in young people. But, this option depends on the moment that patients present themselves. Look, if those patients with a tendon tear have had treatment by the physiotherapist for three months.... well, there is nothing left to do for me anymore, or for the orthopedic surgeon. (...) No, you should prevent discovering this in the chronic phase. That's where it goes wrong. (...) Patients with chronic complaints, they suffered or are still suffering from an inflammation. They 
suffer, of course they suffer. I already know that. I do not need US to figure that out." (GP 2, experienced, low-end US user)

US is often ordered to confirm a diagnosis. Consequently, some GPs adapt US as a logic step in their diagnostic work-up. Some GPs mention inadequate ordering and little added value of US with respect to further treatment. Some GPS use it to satisfy patient expectations and to provide an explanation for the pain, and subsequently better acceptation by patients, which they actually perceive as a kind of treatment.

"If diagnostic imaging tests in an early phase lead to a better prognosis isn't known by me. I do think that it leads to a better acceptation in patients. Also, if the patient accepts his complaint, and finds a way dealing with it, a lot has been gained." (GP 15, experienced, high-end US user)

\section{Interdisciplinary consultation and referral}

Interdisciplinary consultation or referral to orthopedic surgeons or physiotherapists is another strategy when dealing with diagnostic uncertainty. This is sometimes induced by the diagnostic outcome of the US. Some GPs, who consider their diagnostic knowledge insufficient, refer patients to a physiotherapist, irrespective of duration of complaints. GPs refer to orthopedic surgeons in traumatic or chronic cases.

"In case of persisting, intense complaints, with great influence on daily live, I tend to consult the orthopaedic surgeon: Is surgery or another kind of therapy indicated in this patient?"

(GP 8, less experienced, low-end US user)

\section{Discussion}

The aim of this study was to obtain a deeper understanding about GPs perspectives on the diagnostic work-up in patients with shoulder pain. This qualitative study contributes new information to the published literature on explaining GPs diagnostic preferences and uncertainties, and reasons for the variety of diagnostic classifications being used to diagnose shoulder pain. GPs use a variety of diagnostic classifications, with a tendency towards a specific, patho-anatomical diagnosis. GPs have established their individual diagnostic work-ups by using existing tools and motivated deviation. They have to deal with diagnostic uncertainties and use a variety of strategies to deal with these uncertainties, e.g. diagnostic imaging. This study shows no unequivocal approach 
regarding the diagnostic work-up of shoulder pain, and the results do not seem to be related to GP characteristics.

This is the first qualitative study investigating the diagnostic work-up of GPs in patients with shoulder pain. Dutch GPs still tend to prefer more specific diagnoses, even though the shoulder guidelines advise to refrain from using specific diagnoses. ${ }^{3}$ Our findings conflict with previous studies based on medical record review in The Netherlands and UK that show that GPs in most cases record a non-specific diagnosis. ${ }^{5,6}$ This difference may be explained by the difference in study design. In these studies only ICPC or Read codes were retrieved and analysed. Using code systems forces GPs to choose between several codes, and doesn't reflect diagnostic reasoning. In The Netherlands, most GPs add their own diagnosis as free text to their selected ICPC code. Formulating a specific etiological diagnosis is a fundamental part of almost every medical encounter, and helps to target treatment and to earn trust by patients. ${ }^{27}$ The shoulder guideline states that it is more effective to treat simply on the basis of signs and symptoms. It has been illustrated before that a specific etiological diagnosis is not necessarily always an obligatory or even important element in this process. ${ }^{27}$ However, this approach is not preferred by GPs according to our findings.

The lack of uniformity in diagnostic frameworks is not solely confined to clinical practice, but also exists in research. ${ }^{28,29}$ Constant variety in diagnostic classifications may be an important reason why conflicting conclusions are drawn from research evaluating the efficacy of treatments in patients with shoulder pain, and why systematic reviews do not find strong evidence for any of those treatments, since heterogeneity might obscure any effect. ${ }^{29}$

In agreement with other studies, we found several barriers to GPs' experience in using existing tools in the diagnostic work-up of shoulder pain. ${ }^{30-33}$ Although GPs stress the importance of implementing evidence, they experience barriers that limit incorporation of new evidence. For several GPs, current evidence based guidelines seem to conflict with their own clinical expertise and previous experiences. ${ }^{31,32}$ Also, some GPs develop illness scripts in patients with shoulder pain. However, the content of each script depends on the actual experience the GP has with the specific disorder. ${ }^{34}$ GPs have conflicting perceptions on diagnostic imaging tests, despite a clear statement in shoulder guidelines limiting its role to cases in which conservative treatment fails or red flag situations. ${ }^{3,11-13}$ In agreement with previous research, gaining trust and patient reassurance are reasons mentioned by GPs for ordering US. ${ }^{35,36}$ However, there is limited evidence for the assumption that diagnostic tests actually intrinsically reassure patients, as other factors (e.g. doctor-patient relation, pre-test information, and individual fears) must be taken into account. ${ }^{35}$ Diagnostic uncertainty is a typical general practice characteristic, and gut feelings, invoking a 'sense of alarm', are often 
used by GPs to support their diagnostic reasoning. ${ }^{37}$ This study shows that also in the diagnostic work-up of patients with shoulder pain, gut feelings play a role.

The main strength of our study is that it explains GPs diagnostic preferences and uncertainties. The results are not surprising and sound logical. We obtained a wide range of views in a diverse group of Dutch GPs. As a result of our sampling method, findings are more likely to fit samples and settings beyond those of this particular study. ${ }^{23}$ Moreover, the setting of Dutch GPs, and their open access to US, corresponds with the setting in the UK and most Nordic countries (Denmark, Sweden, Norway, Finland and Iceland), which improves transferability of our results. Furthermore, rigorous qualitative methods were applied to data collection while analysis happened in an iterative process. Data collection continued until stable (sub)categories emerged and saturation was reached. ${ }^{22} \mathrm{~A}$ limitation of this study is that this study was restricted to interviews. No observations of patient encounters were made to collect additional data. We tried to compensate for this by asking GPs to recall their last patient encounter, and by asking for examples to illustrate statements.

Our study shows that there are many reasons why GPs use different classifications to diagnose shoulder pain, with variations between GPs dealing with diagnostic uncertainties. As diagnostic classifications are a guide to treatment decisions, unsatisfactory reproducibility also might affect treatment outcome. Although GPs are advised to establish a non-specific diagnosis, our results imply GPs' need for a more specific diagnosis. Research suggests that US can have a positive impact on establishing a more accurate diagnosis in patients with shoulder complaints and on guiding them through primary and secondary care. ${ }^{38}$ US has the advantage that the results can tailor therapy, which might improve patient outcome. However, so far there is no evidence to support this strategy. The results of an ongoing randomised controlled trial investigating the cost-effectiveness of applying US to the diagnostic work-up, will give us more information about this strategy. ${ }^{39}$ Until then, GPs are encouraged to follow the current shoulder guidelines. Another strategy would be one where local GPs, physiotherapists and orthopedic surgeons improve their collaboration and communication. A promising development is the implementation of GPs with a special interest in musculoskeletal disorders. These trained GPs can act as consultant and initiate the above mentioned collaborations.

Further research among GPs and patients investigating classification preferences is advised. Also, results from research about the role of US in (sub)acute and chronic shoulder pain would enhance GPs' decision-making. Although shoulder guidelines are available, Dutch GPs seem to prefer their own diagnostic work-up procedure. Hence, we advise to train GPs how to combine research evidence, their own experience and 
expertise, and their patients' preferences in decision-making concerning diagnostic work-up. Guideline authors should take our findings into account. As other factors influence patient's reassurance, GPs might benefit from training in tolerating diagnostic uncertainty, and the use of other reassuring strategies. Consequently, US will be more adequately applied. 


\section{References}

1. Feleus A, Bierma-Zeinstra SM, Miedema HS, Bernsen RM, Verhaar JA, Koes BW. Incidence of nontraumatic complaints of arm, neck and shoulder in general practice. Man Ther. 2008;13:426-433.

2. Picavet HS, Schouten JS. Musculoskeletal pain in the Netherlands: prevalences, consequences and risk groups, the DMC(3)-study. Pain. 2003;102:167-178.

3. Winters JC, van der Windt DAWM, Spinnewijn WEM, et al. Shoulder pain guideline of the Dutch College of General Practitioners (in Dutch). Huisarts Wet. 2008;51:555-565.

4. van der Windt DA, Koes BW, Boeke AJ, Devillé W, De Jong BA, Bouter LM. Shoulder disorders in general practice: prognostic indicators of outcome. Br J Gen Pract. 1996;46:519-523.

5. Dorrestijn O, Greving K, van der Veen WJ, et al. Patients with shoulder complaints in general practice: consumption of medical care. Rheumatology. 2011;50:389-395.

6. Linsell L, Dawson J, Zondervan K, et al. Prevalence and incidence of adults consulting for shoulder conditions in UK primary care; patterns of diagnosis and referral. Rheumatology. 2006;45:215-221.

7. de Winter AF, Jans MP, Scholten RJ, Deville W, van Schaardenburg D, Bouter LM. Diagnostic classification of shoulder disorders: interobserver agreement and determinants of disagreement. Ann Rheum Dis. 1999;58:272-277.

8. Groenier $\mathrm{KH}$, Winters JC, de Jong BM. Classification of shoulder complaints in general practice by means of nonmetric multidimensional scaling. Arch Phys Med Rehabil. 2003;84:812-817.

9. De Jongh AC. Shoulder complaints in general practice (Dissertation). Rotterdam, Erasmus University Rotterdam; 1994.

10. Groenier KH, Winters JC, van Schuur WH, De Winter AF, Meyboom-De Jong B. A simple classification system was recommended for patients with restricted shoulder or neck range of motion. J Clin Epidemiol. 2006;59:599-607.

11. NHS/NICE CKSb. Shoulder pain guideline Available at: http://www.cks.nhs.uk/shoulder_pain.

12. Group AAMPG. Evidence-Based Management of Acute Musculoskeletal Pain. Available at: www.nhmrc.gov.au/_files_nhmrc/publications/attachments/cp95.pdf. (2003).

13. Robb G, Arroll B, Goodyear-Smith F. Summary of an evidence-based guideline on soft tissue shoulder injuries and related disorders-Part 1: Assessment. J Primary Health Care 2009;1:36-41.

14. Britt H, Miller GC, Knox S. Shoulder syndrome. In imaging orders by general practitioners in Australia 1999-2000. AlHW Cat. No. GEP 7. General Practice Series No. 7. Available at: www.aihw.gov.au/publications/index.cfm/title/6949 (2001).

15. Broadhurst NA, Gialamis A, McElroy HJ, Bielby JJ. How do Australian GPs manage shoulder dysfunction? Aust Fam Physician. 2004;33:861-864.

16. Buchbinder R, Staples MP, Shanahan EM, Roos JF. General practitioner management of shoulder pain in comparison with rheumatologist expectation of care and best evidence: an Australian national survey. PLoS One. 2013;8:e61243.

17. Masters S, O'Doherty L, Mitchell GK, Yelland M. Acute shoulder pain in primary care - an observational study. Aust Fam Physician. 2007;36:473-476.

18. Loebenberg MI, Rosen JE, Ishak C, Jazrawi LM, Zuckerman JD. A survey of decision-making processes in the treatment of common shoulder ailments among primary care physicians. Bull Hosp Jt Dis. 2006;63:137-144.

19. Broadhurst N, Baghurst T, MacLaren S. Ultrasound imaging for shoulder pain in general practice. Aust Fam Physician. 2004;33:668-669.

20. Ottenheijm RPG, van 't Klooster IGM, Starmans LMM, et al. Ultrasound-diagnosed disorders in shoulder patients in daily general practice: a retrospective observational study. BMC Fam Pract. 2014;15:115

21. Britten N. Qualitative interviews in medical research. BMJ. 1995;311:251-253.

22. Kuper A, Lingard L, Levinson W. Critically appraising qualitative research. BMJ. 2008; 337:a1035.

23. Flick U. An introduction to qualitative research. Fourth edition ed: SAGE Publications; 2009.

24. Central Committee on Research Involving Human Subjects. Available at: www.ccmo-online.nl/en/helpme-on-my-way (2014)

25. Strauss A CJ. Basics of qualitative research. Thirth edition ed: London: SAGE Publications; 2008. 
26. Haynes RB, Sackett DL, Guyatt GH, Cook DJ, Gray JA. Transferring evidence from research into practice: 4. Overcoming barriers to application. ACP J Club. 1997; 126:A14-15.

27. Dinant GJ, Buntinx FF, Butler CC. The necessary shift from diagnostic to prognostic research. BMC Fam Pract. 2007;8:53.

28. Green S, Buchbinder R, Glazier R, Forbes A. Systematic review of randomised controlled trials of interventions for painful shoulder: selection criteria, outcome assessment, and efficacy. BMJ. 1998;316:354-360.

29. Schellingerhout JM, Verhagen AP, Thomas S, Koes BW. Lack of uniformity in diagnostic labeling of shoulder pain: time for a different approach. Man Ther. 2008;13:478-483.

30. Cabana MD, Rand CS, Powe NR, et al. Why don't physicians follow clinical practice guidelines? A framework for improvement. JAMA. 1999;282:1458-1465.

31. Lugtenberg M, Burgers JS, Besters CF, Han D, Westert GP. Perceived barriers to guideline adherence: a survey among general practitioners. BMC Fam Pract. 2011;12:98.

32. Zwolsman S, te Pas E, Hooft L, Wieringa-de Waard M, van Dijk N. Barriers to GPs' use of evidence-based medicine: a systematic review. Br J Gen Pract. 2012;62:e511-521.

33. Lugtenberg M, Zegers-van Schaick JM, Westert GP, Burgers JS. Why don't physicians adhere to guideline recommendations in practice? An analysis of barriers among Dutch general practitioners. Implement Sci. 2009;4:54.

34. Custers $\mathrm{E}$, Boshuizen $\mathrm{H}$, Schmidt $\mathrm{H}$. The role of illness scripts in the development of medical diagnostic expertise: results from an interview study. Cognition and Instruction. 1998;16:367-398.

35. van Ravesteijn $H$, van Dijk I, Darmon $D$, et al. The reassuring value of diagnostic tests: a systematic review. Patient Educ Couns. 2011;86:3-8.

36. Hale JP, Cohen DR, Robling MR, Houston H, Kinnersley $\mathrm{P}$, Hourihan MD. Multi-disciplinary research can be more than parallel-disciplinary research: the case of GPs' use of magnetic resonance imaging. Fam Pract. 1999;16:301-304.

37. Stolper CF, Van de Wiel MW, De Vet HC, et al. Family physicians' diagnostic gut feelings are measurable: construct validation of a questionnaire. BMC Fam Pract. 2013;14:1.

38. Ottenheijm RPG, Jansen MJ, Staal JB, et al. Accuracy of diagnostic ultrasound in patients with suspected subacromial disorders: a systematic review and meta-analysis. Arch Phys Med Rehabil. 2010;91: 1616-1625.

39. Ottenheijm RP, Joore MA, Walenkamp GH, et al. The Maastricht Ultrasound Shoulder pain Trial (MUST): ultrasound imaging as a diagnostic triage tool to improve management of patients with non-chronic shoulder pain in primary care. BMC Musculoskelet Disord. 2011;12:154. 


\section{Chapter 4}

Ultrasound-diagnosed disorders in shoulder patients

in daily general practice:

a retrospective observational study

Ramon PG Ottenheijm, Inge GM van 't Klooster, Laurens MM Starmans, Kurt Vanderdood, Rob A de Bie, Geert-Jan Dinant, Jochen WL Cals

BMC Fam Pract 2014;15:115 


\section{Abstract}

\section{Background}

Ultrasound imaging (US) is considered an accurate and widely available method to diagnose subacromial disorders. Yet, the frequency of the specific US-diagnosed shoulder disorders of patients with shoulder pain referred from general practice is unknown. We set out to determine the frequency of specific US-diagnosed shoulder disorders in daily practice in these patients and to investigate if the disorders detected differ between specific subgroups based on age and duration of pain.

\section{Methods}

A predefined selection of 240 ultrasound reports of patients with shoulder pain (20 reports for each month in 2011) from a general hospital (Orbis Medical Centre Sittard-Geleen, The Netherlands) were descriptively analysed. Inclusion criteria were: (i) referral from general practice, (ii) age $\geq 18$ years, and (iii) unilateral shoulder examination. Subgroups were created for age ( $<65$ years and $\geq 65$ years) and duration of pain (acute or subacute ( $<12$ weeks) and chronic ( $\geq 12$ weeks)). The occurrence of each specific disorder is expressed as absolute and relative frequencies.

\section{Results}

With $29 \%$, calcific tendonitis was the most frequently diagnosed disorder, followed by subacromialsubdeltoid bursitis (12\%), tendinopathy (11\%), partial-thickness tears (11\%), full-thickness tears (8\%) and ACosteoarthritis $(0.4 \%)$. For $40 \%$ of patients, no disorders were found on US. Significantly more full thicknesstears were found in the $\geq 65$ years group. 'No disorders' was reported significantly more often in the $<65$ years group. The supraspinatus tendon was the most frequently affected tendon $(72 \%)$.

\section{Conclusions}

Calcific tendonitis is the most common US-diagnosed disorder affecting patients in general practice, followed by subacromial-subdeltoid bursitis, tendinopathy, partial- and full-thickness tears and AC-osteoarthritis. Fullthickness tears were diagnosed significantly more frequently in patients $\geq 65$ years, while 'no disorders' was more frequently reported in patients $<65$ years. Our findings imply that patients can be stratified into diagnostic subgroups, allowing more tailored treatment than currently applied. 


\section{Background}

Shoulder pain is a common and disabling complaint in general practice with often a poor prognosis. ${ }^{1-4}$ Almost $90 \%$ of patients with shoulder pain are diagnosed and treated in general practice, while only $10 \%$ are referred for a specialist opinion. ${ }^{5}$ Accurately diagnosing patients with shoulder pain is, however, a complex problem. Tailoring treatment to the underlying disorder is difficult for general practitioners (GPs), as the medical history and physical examination do not provide conclusive evidence on the patho-anatomical origin of the symptoms. ${ }^{1}$ Of the currently available additional diagnostic imaging tests, ultrasound imaging (US) is considered an accurate and widely available method to diagnose subacromial disorders. ${ }^{6-9}$

Subacromial disorders are considered the most common pathology affecting the shoulder. The spectrum of subacromial pathology includes rotator cuff tendinopathy (tendinosis), calcific tendonitis, partial- and full-thickness tears, and subacromialsubdeltoid (SASD) bursitis. ${ }^{10-12}$ Prevalence studies of US diagnoses in patients with shoulder pain in secondary care have shown prevalences ranging from $30-39 \%$ for tendinopathy, $13-15 \%$ for calcific tendonitis, $13-51 \%$ for partial-thickness tears, $24-70 \%$ for full-thickness tears, and $12-56 \%$ for SASD bursitis. ${ }^{6}$ Remarkably, the prevalence of these disorders in general practice is still unknown. Unravelling the current case mix of patients with shoulder pain seen in general practice should give GPs more insight into the underlying causes of shoulder pain. The first step in providing this insight is to evaluate current daily practice.

The primary objective of this study was to determine the frequency of the specific USdiagnosed shoulder disorders of patients with shoulder pain referred from general practice. Additionally, we investigated which tendons (rotator cuff and longhead of the biceps) were affected, and if the frequencies differed between specific subgroups based on age and duration of pain.

\section{Methods}

We conducted a retrospective observational study on a selection of reports of US shoulder examinations performed at the Orbis Medical Centre (OMC), a general hospital in the south of the Netherlands, in 2011. The OMC's catchment area includes approximately 80 GPs (approximately 200,000 people) who refer their patients for US of the shoulder to this hospital through direct access. The study was approved by the Atrium - Orbis - Zuyd Medical Ethics Committee. Informed consent from patients was 
not needed as this was a retrospective chart study and data were analysed anonymously.

The OMC radiology department annually performs approximately 2400 US examinations of the shoulder for general practice patients. We expected a frequency of $24 \%$ for calcific tendonitis, based on the only available study with patients recruited from primary care. ${ }^{13}$ Based on a $95 \%$ confidence interval and a precision of $5 \%$, this resulted in a required sample size of $252 .{ }^{14}$ We therefore selected a predefined sample of $10 \%$ of the total of 2400 reports from the OMC database, stratified by month (January - December) to ensure equal distribution across seasons, resulting in a total sample of 240 reports. As degeneration is one of the mechanisms leading to rotator cuff pathology, and therefore a potential confounder, we decided also to stratify for age. Since approximately $10 \%$ of all shoulder US examinations are performed on patients $\geq 65$ years, we decided that $10 \%$ of all reports per month had to be of patients $\geq 65$ years. Hence, we analyzed the first 18 reports of each month of patients $<65$ years and the first two reports of patients $\geq 65$ years. We realise that degeneration is a continuum, and evidence for a clear cut-off value for age as a confounder is lacking. Although arbitrary, we think it is plausible to set this cut-off for age at 65 years. General inclusion criteria were: (i) patients with shoulder pain referred by their GP (ii) age $\geq 18$ years, and (iii) unilateral shoulder examination.

All US examinations were performed by an ultrasonographer with $>15$ years of experience of shoulder US, using an international scanning protocol, ${ }^{15}$ and standardised US diagnostic criteria for pathology were used. ${ }^{16}$ The long head of the biceps tendon (LHBT), rotator cuff, SASD bursa, deltoid muscle and acromioclavicular (AC)-joint were routinely evaluated. US examinations were performed using equipment manufactured by two vendors; Hitachi/Aloka and Philips. All units were equipped with $5-12 \mathrm{MHz}$ broadband linear-array transducers.

Two researchers (IGMK and LMMS) independently assessed the US reports, including age, gender and affected shoulder, and each verified the data entered by the other. The first 32 cases were assessed with a third independent researcher (RPGO) to discuss and enhance consistent assessments. Disagreements between the two main assessors were discussed and solved by consensus. In cases of persistent disagreement (14 of 240 cases), the third rater resolved the disagreement. Relevant data were missing from five US reports. These were completed by contacting the ultrasonographer.

For the subsequent analyses, subgroups were created for age ( $<65$ years and $\geq 65$ years) and duration of complaints (acute or subacute ( $<12$ weeks) and chronic ( $\geq 12$ weeks)), based on the duration indicated by the GP in the letter of referral. We used 12 weeks as the cut-off value, as the guidelines for shoulder complaints of the Dutch College of General Practitioners recommend US of the shoulder only in case of complaints 
persisting despite conservative treatment. ${ }^{1}$ Normally, this refers to a period of approximately 12 weeks. All phrases like 'a couple' and 'some' were consistently interpreted and transformed into duration of pain in weeks, e.g. 'a couple of weeks' into two weeks, and 'chronic' into $\geq 12$ weeks.

We calculated proportions with corresponding 95\% confidence intervals. The chi-squared test was used for subgroup analysis. Data were analysed using SPSS (version 19.0).

\section{Results}

\section{Patient characteristics}

Of the 240 included patients, 131 (55\%) were women. Mean patient age was 51.5 years (SD 12.1, range 20-83 years). US was performed on the left shoulder in 125 (52\%) patients and on the right shoulder in 115 (48\%). In six US reports the ultrasonographer commented that US was less reliable due to adiposity (3) or restriction of movement (3). Duration of pain was mentioned in $33 \%$ of the reports (79/240), with a minimal duration of 1 week and a maximum of $>3$ years. The duration referred to acute or subacute complaints in $42 \%$ (33/79) of these reports, and to chronic complaints in $58 \%$ (46/79). Median duration was 12 weeks, with lower and upper quartiles of 6 and 14 weeks.

\section{Frequency of US-diagnosed shoulder disorders}

In $40 \%$ of the patients (97/240), no disorders were found on US. Of the remaining $60 \%$ of the patients (143/240), multiple disorders were found in $40 \%$ (59/143). Of these, 41 patients had two disorders, 15 had three, two had four and one patient had five disorders described in the US report.

Table 4.1 shows the frequency of the diagnosed disorders. With $29 \%$, calcific tendonitis was the most frequently diagnosed disorder (69/240), followed by SASD bursitis (12\%, $29 / 240)$, tendinopathy $(11 \%, 27 / 240)$, partial-thickness tears $(11 \%, 27 / 240)$, fullthickness tears $(8 \%, 20 / 240)$ and AC-osteoarthritis $(0.4 \%, 1 / 240)$. Of the remaining patients with a disorder, $8 \%(18 / 240)$ had a diagnosis classified as 'Other' (enthesophyte (5), tenosynovitis of the LHBT (4), fracture of the greater tuberosity (2), AC-joint widening (2), post-op supraspinatus tendon repair (2), subluxation of the LHBT (1) and calcific deposit in deltoid muscle(1)). 


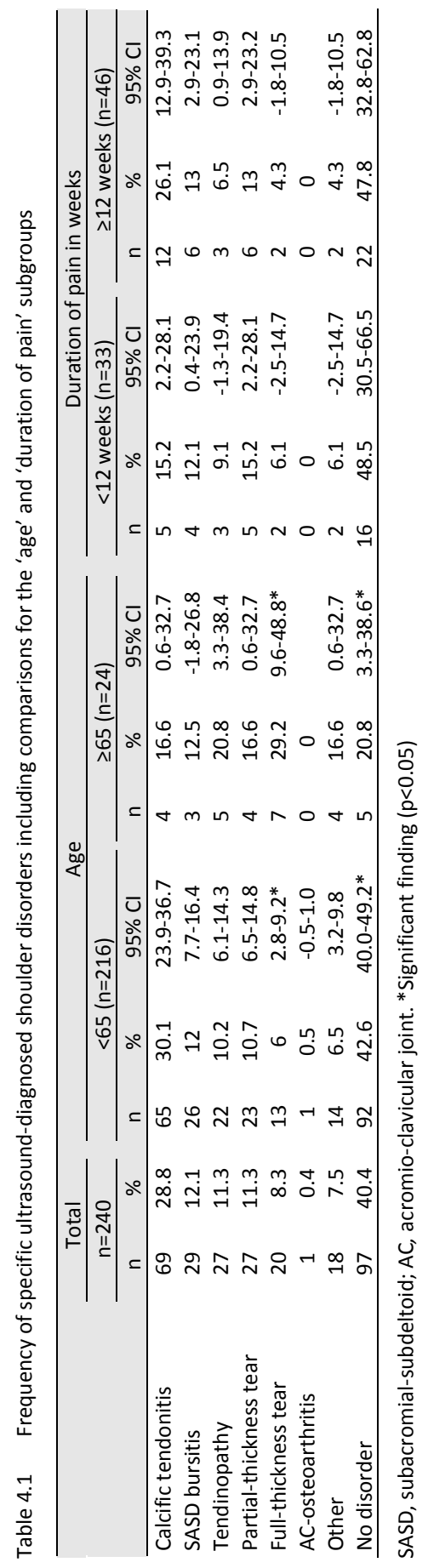


Within the group of 41 patients with two disorders, calcific tendonitis was the most prevalent disorder $(56 \%, 23 / 41)$, followed by tendinopathy $(15 \%, 6 / 41)$. Calcific tendonitis was most often seen in combination with tendinopathy (34\%, 14/41), followed by SASD bursitis $(15 \%, 6 / 41)$.

Table 4.1 shows that significantly more full-thickness tears were found in the $\geq 65$ years group, while 'No disorders' was reported significantly more often in the $<65$ years group. The frequency of the other specific disorders did not differ significantly between the subgroups.

\section{Tendons affected}

As multiple disorders per patient could be recorded, the total number of affected tendons was 183 (Table 4.2). The supraspinatus tendon was the most frequently affected tendon $(72 \% 132 / 183)$, followed by the subscapularis tendon $(12 \%)$, infraspinatus tendon (12\%) and the LHBT (4\%). Forty-three percent (10/29) of patients with SASD bursitis had concomitant calcific tendonitis, with a specific calcific bursitis in $10 \%(3 / 29)$.

Table 4.2 Tendon location of different disorders

\begin{tabular}{|c|c|c|c|c|c|c|c|c|c|c|c|c|}
\hline & \multicolumn{2}{|c|}{$\begin{array}{c}\text { Calcific } \\
\text { tendonitis } \\
n=94\end{array}$} & \multicolumn{2}{|c|}{$\begin{array}{c}\text { Tendinopathy } \\
\text { n=28 } \\
\end{array}$} & \multicolumn{2}{|c|}{$\begin{array}{c}\text { Partial-thickness } \\
\text { tear } \\
\mathrm{n}=28 \\
\end{array}$} & \multicolumn{2}{|c|}{$\begin{array}{c}\text { Full-thickness } \\
\text { tear } \\
\mathrm{n}=22 \\
\end{array}$} & \multicolumn{2}{|c|}{$\begin{array}{c}\text { Other } \\
\text { disorder } \\
n=11\end{array}$} & \multicolumn{2}{|c|}{$\begin{array}{l}\text { Total tendons } \\
\text { n=183 }\end{array}$} \\
\hline & $n$ & $\%$ & $n$ & $\%$ & $n$ & $\%$ & $n$ & $\%$ & $\mathrm{n}$ & $\%$ & $\mathrm{n}$ & $\%$ \\
\hline SSP & 58 & 61.7 & 26 & 92.9 & 26 & 92.9 & 17 & 77.3 & 5 & 45.5 & 132 & 72.1 \\
\hline ISP & 19 & 20.2 & 0 & 0 & 1 & 3.6 & 1 & 4.5 & 1 & 0.9 & 22 & 12.0 \\
\hline SSC & 17 & 18.1 & 1 & 3.6 & 1 & 3.6 & 3 & 13.6 & 0 & 0 & 22 & 12.0 \\
\hline LHBT & 0 & 0 & 1 & 3.6 & 0 & 0 & 1 & 4.5 & 5 & 45.5 & 7 & 3.8 \\
\hline
\end{tabular}

SSP, supraspinatus tendon; ISP, infraspinatus tendon; SSC, subscapularis tendon; LHBT, long head biceps tendon

\section{Discussion}

\section{Main findings}

This study is the first to describe specific shoulder disorders detected on US in general practice patients who are symptomatic enough and have had symptoms for a sufficient duration of time to warrant referral by their GP for US. Calcific tendonitis, also referred to as calcific tendinopathy, was the most common US-diagnosed disorder affecting these general practice patients, followed by SASD bursitis, tendinopathy, partial- and 
full-thickness tears and AC-osteoarthritis. We found that full-thickness tears were diagnosed more frequently in patients $\geq 65$ years, while patients $<65$ years were more often reported to have 'no disorders'. Prevalence of specific shoulder disorders did not differ between patients with acute or subacute complaints or chronic pain. The supraspinatus tendon was the most commonly affected tendon.

\section{Strengths and limitations of this study}

This study was the first to describe specific shoulder disorders detected on US in general practice patients. As almost $90 \%$ of the patients with shoulder pain are diagnosed and treated in general practice, ${ }^{5}$ these results are useful in the debate about the role of US in the diagnostic work-up of patients and about tailoring treatment to the underlying disorder, as well as in designing new studies.

We carried out a retrospective observational study on a selection of $10 \%(n=240)$ of the US reports made in one year. The retrospective nature as well as the size of our sample and sampling method may have caused bias. As daily practice was the scope of our study, we only studied those patients for whom the GP ordered US. We realise that our secondary subgroup analyses were probably underpowered, especially for the 'duration of pain' subgroups, as duration was mentioned in only $33 \%$ of the reports. This may also have caused some selection and measurement bias limiting the interpretations. However, we can only speculate about the reasons for not reporting duration. Although the reliability of US examinations by a single ultrasonographer can be questioned, this situation is representative of daily practice. As the interrater-reliability between ultrasonographers examining patients with shoulder pain is high, ${ }^{17,18}$ a predefined scanning protocol was used, and the ultrasonographer was unaware of this planned study, the additional value of a second independent ultrasonographer would have been doubtful. Dynamic examinations to reveal impingement or adhesive capsulitis were not performed by the ultrasonographer. This might have resulted in an under representation of adhesive capsulitis given the three patients with a restricted range of motion. To enhance consistent assessment, two researchers independently assessed the US reports and each verified the data entered by the other researcher. To correct for seasonal influences, e.g. traumatic rotator cuff tears in winter, we selected 20 reports from each month.

\section{Comparison with existing literature}

Our results are not in line with previous secondary care studies, which showed lower prevalence of calcific tendonitis and higher prevalence of bursitis and the degenerative disorders tendinopathy and tendon tears. ${ }^{6,7}$ This difference may be explained by the 
difference in patient setting and the resulting case mix. Previous studies were conducted in patients with mostly chronic shoulder pain consulting in secondary care settings, mostly in the diagnostic work-up for surgery. Referral to secondary care in the Netherlands is done by GPs, and patients are typically only referred for US in case of persistent complaints despite conservative treatment. Another explanation could be an overrepresentation in our study of patients with acute flairs of calcific tendonitis. This results in a different case mix compared to secondary care, where tendon tears are most prevalent.

The only study conducted in a primary care setting also found calcific tendonitis to be the most prevalent disorder (24\%). ${ }^{13}$ However, that study found more partial-thickness tears (23\%) and fewer patients with no disorder detected on US (15\%). This can be explained by the difference in study design, patient setting and patient characteristics; the study was part of a wider prospective diagnostic accuracy study, with $98 \%$ of the patients recruited from physiotherapy practice and $93 \%$ of the recruited patients involved in a liability procedure because of an accident.

Our results show that the supraspinatus tendon was the most commonly affected tendon. This is in agreement with previous findings ${ }^{13}$ and the general belief that the supraspinatus tendon is the tendon most commonly involved in rotator cuff disease. ${ }^{19}$

\section{Implications for practice and research}

As US is an easily accessible, accurate, relatively cheap and non-invasive and nonionising imaging procedure, GPs in the study area frequently refer patients for US, resulting in a rise in test volume of $100 \%$ over the last 6 years. However, at present there is no clear evidence for using US for acute shoulder pain. The present study shows that different subacromial disorders can be detected in patients with shoulder pain in general practice. This implies that patients can be stratified into diagnostic subgroups, allowing more tailored treatment than currently applied. In general practice patients with low back pain, a stratified management approach combining prognostic screening and treatment targeting was found to improve patient outcome. ${ }^{20}$ In daily practice, combining clinical information with US findings could potentially be helpful to tailor the treatment of patients with shoulder pain. It is likely that patients will respond best to interventions that address the etiology, affected structures, impairments, and relevant biomechanics that are specific to their diagnosis. However, there is no current general practice evidence to back this up. ${ }^{16}$

Since a certain percentage of the US findings found is not responsible for the reported complaints, ${ }^{21,22}$ GPs should realise that there might not be a straightforward correlation between US and clinical findings. A prospective study of US findings with clinical input is 
necessary to provide a more robust indication of the true prevalence in general practice.

Calcific tendonitis was found to be the most prevalent disorder in our study, affecting about one-third of all patients. Calcific tendonitis of the shoulder is a common painful disorder characterised by calcifications in rotator cuff tendons, resulting in repeating painful episodes. The pathogenesis is still a matter of controversy. The most dominant view states that the deposition of calcium crystals within the substance of the tendon is not a degenerative process but one that is actively mediated by cells in a viable tendon. A natural cycle of repair exists, but this cycle can be blocked or delayed. ${ }^{23-25}$ About $50 \%$ of patients with rotator cuff calcifications have shoulder pain, meaning that many individuals with radiographic evidence of calcific tendonitis are asymptomatic. It can be helpful if US reports contain information about size and location, as well as morphologic shape of the calcifications, as this might predict symptomatology. For instance, larger calcifications, those located at the confluence of supraspinatus and infraspinatus tendons, and calcifications in the resorptive phase tend to be more symptomatic. ${ }^{23,25}$ Given this natural recovery cycle and asymptomatic patients, the question arises if all patients in our study with calcific tendonitis actually suffered from these calcification deposits and needed treatment. It is unknown if the same goes for the other disorders, especially in patients with more than one disorder.

By contrast, $40 \%$ of the patients in our study had no disorders detected on US. It is not surprising that the majority of these patients (95\%) were $<65$ years. Hence, GPs may use US to diagnose disorders, but also to rule out specific shoulder disorders. In comparison to the elderly, younger patients are more physically active and may therefore experience more disability when faced with pain. It is our experience that younger patients with chronic shoulder pain in particular often ask for imaging to ensure that no diagnosis is missed. One should keep in mind that shoulder pain can also be caused by referred pain. Also, it is to be expected that tears of the rotator cuff occur more frequently in elderly patients, as a result of age-related degenerative tendon changes.

\section{Conclusions}

This study shows that, contrary to findings from secondary care, calcific tendonitis is the most common US diagnosed disorder affecting patients who are symptomatic enough and have had symptoms for a sufficient duration to warrant referral by their GP for US. Full-thickness tears were diagnosed more frequently in patients $\geq 65$ years. Our findings imply that patients can be stratified into diagnostic subgroups, allowing more tailored treatment than currently applied. 


\section{References}

1. Winters JC, van der Windt DAWM, Spinnewijn WEM, et al. Shoulder pain guideline of the Dutch College of General Practitioners (in Dutch). Huisarts Wet. 2008;51:555-565.

2. Feleus A, Bierma-Zeinstra SM, Miedema HS, Bernsen RM, Verhaar JA, Koes BW. Incidence of nontraumatic complaints of arm, neck and shoulder in general practice. Man Ther. 2008;13:426-433.

3. Picavet HS, Schouten JS. Musculoskeletal pain in the Netherlands: prevalences, consequences and risk groups, the DMC(3)-study. Pain. 2003;102:167-178.

4. Bot SD, van der Waal JM, Terwee CB, et al. Incidence and prevalence of complaints of the neck and upper extremity in general practice. Ann Rheum Dis. 2005;64:118-123.

5. van der Windt DA, Koes BW, Boeke AJ, Devillé W, De Jong BA, Bouter LM. Shoulder disorders in general practice: prognostic indicators of outcome. Br J Gen Pract. 1996;46:519-523.

6. Ottenheijm RPG, Jansen MJ, Staal JB, et al. Accuracy of diagnostic ultrasound in patients with suspected subacromial disorders: a systematic review and meta-analysis. Arch Phys Med Rehabil. 2010;91: 1616-1625.

7. Dinnes J, Loveman E, Mclntyre L, Waugh N. The effectiveness of diagnostic tests for the assessment of shoulder pain due to soft tissue disorders: a systematic review. Health Technol Assess. 2003;7:1-166.

8. de Jesus JO, Parker L, Frangos AJ, Nazarian LN. Accuracy of MRI, MR arthrography, and ultrasound in the diagnosis of rotator cuff tears: a meta-analysis. Am J Roentgenol. 2009;192:1701-1707.

9. Smith TO, Back T, Toms AP, Hing CB. Diagnostic accuracy of ultrasound for rotator cuff tears in adults: a systematic review and meta-analysis. Clin Radiol. 2011;66:1036-1048.

10. Lewis J, Tennent D, eds. How effective are diagnostic tests for the assessment of rotator cuff disease of the shoulder? London: Blackwell, BMJ Books; 2007. MacAuley D, Best TM, eds. Evidence based sports medicine.

11. Baring T, Emery RJH, Reilly P. Management of rotator cuff disease: specific treatment for specific disorders. Best Prac Res Clin Rheumatol. 2007;21:279-294.

12. Riley G. Tendinopathy - from basic science to treatment. Nat Clin Pract Rheumatol. 2008;4:82-89.

13. Cadogan A, Laslett M, Hing WA, McNair PJ, Coates MH. A prospective study of shoulder pain in primary care: prevalence of imaged pathology and response to guided diagnostic blocks. BMC Musculoskelet Disord. 2011;12:119.

14. Samplesize. Available at: http://sampsize.sourceforge.net/iface/

15. Beggs I, Bianchi S, Bueno A. Musculoskeletal Ultrasound Technical Guidelines. 1 Shoulder. Available at http://www.essr.org/html/img/pool/shoulder.pdf

16. Ottenheijm RP, Joore MA, Walenkamp GH, et al. The Maastricht Ultrasound Shoulder pain Trial (MUST): ultrasound imaging as a diagnostic triage tool to improve management of patients with non-chronic shoulder pain in primary care. BMC Musculoskelet Disord. 2011;12:154.

17. Le Corroller T, Cohen M, Aswad R, Pauly V, Champsaur P. Sonography of the painful shoulder: role of the operator's experience. Skeletal Radiol. 2008;37:979-986.

18. Rutten MJ, Jager GJ, Kiemeney LA. Ultrasound detection of rotator cuff tears: observer agreement related to increasing experience. Am J Roentgenol. 2010;195:W440-446.

19. Mochizuki T, Sugaya H, Uomizu M, et al. Humeral insertion of the supraspinatus and infraspinatus. New anatomical findings regarding the footprint of the rotator cuff. J Bone Joint Surg Am. 2008;90:962-969.

20. Hill JC, Whitehurst DG, Lewis M, et al. Comparison of stratified primary care management for low back pain with current best practice (STarT Back): a randomised controlled trial. Lancet. 2011;378: 1560-1571.

21. Reilly P, Macleod I, Macfarlane R, Windley J, Emery RJH. Dead men and radiologists don't lie: a review of cadaveric and radiological studies of rotator cuff tear prevalence. Ann R Coll Surg Engl. 2006;88: 116-121.

22. Sher JS, Uribe JW, Posada A, Murphy BJ, Zlatkin MB. Abnormal findings on magnetic resonance images of asymptomatic shoulders. J Bone Joint Surg Am. 1995;77:10-15.

23. Bureau NJ. Calcific tendinopathy of the shoulder. Semin Musculoskelet Radiol. 2013;17:80-84. 
24. Carcia CR, Scibek JS. Causation and management of calcific tendonitis and periarthritis. Curr Opin Rheumatol. 2013;25:204-209.

25. Gosens T, Hofstee DJ. Calcifying tendinitis of the shoulder: advances in imaging and management. Curr Rheumatol Rep. 2009;11:129-134. 


\section{Chapter}

The Maastricht Ultrasound Shoulder pain trial

(MUST): Ultrasound imaging as a diagnostic triage tool to improve management of patients with non-chronic shoulder pain in primary care

Ramon PG Ottenheijm, Manuela A Joore, Geert HIM Walenkamp, René E Weijers, Bjorn Winkens, Jochen WL Cals, Rob A de Bie, Geert-Jan Dinant 


\section{Abstract}

\section{Background}

Subacromial disorders are considered to be one of the most common pathologies affecting the shoulder. Optimal therapy for shoulder pain (SP) in primary care is yet unknown, since clinical history and physical examination do not provide decisive evidence as to the patho-anatomical origin of the symptoms. Optimal decision strategies can be furthered by applying ultrasound imaging (US), an accurate method in diagnosing $\mathrm{SP}$, demonstrating a clear relationship between diagnosis and available therapies. Yet, the clinical costeffectiveness of applying US in the management of SP in primary care has not been studied. The aim of this paper is to describe the design and methods of a trial assessing the cost-effectiveness of ultrasound imaging as a diagnostic triage tool to improve management of primary care patients with non-chronic shoulder pain.

\section{Methods/design}

This randomised controlled trial (RCT) will involve 226 adult patients with suspected subacromial disorders recruited by general practitioners. During a Qualification period of two weeks, patients receive care as usual as advised by the Dutch College of General Practitioners, and patients are referred for US. Patients with insufficient improvement qualify for the RCT. These patients are then randomly assigned to the intervention or the control group. The therapies used in both groups are the same (corticosteroid injections, referral to a physiotherapist or orthopedic surgeon) except that therapies used in the intervention group will be tailored based on the US results. Ultrasound diagnosed disorders include tendinopathy, calcific tendinitis, partial and full thickness tears, and subacromial bursitis. The primary outcome is patient-perceived recovery at 52 weeks, using the Global Perceived Effect questionnaire. Secondary outcomes are disease specific and generic quality of life, cost-effectiveness, and the adherence to the initial applied treatment. Outcome measures will be assessed at baseline, 13, 26, 39 and 52 weeks after inclusion. An economic evaluation will be performed from both a health care and societal perspective with a time horizon of 52 weeks.

\section{Discussion}

The results of this trial will give unique evidence regarding the cost-effectiveness of US as a diagnostic triage tool in the management of SP in primary care. 


\section{Background}

With up to 100 new patient encounters per general practitioner (GP) per year, and a prevalence of $17-20 \%$ in the general population, shoulder pain (SP) is a common and sizable problem in primary care. ${ }^{1-4}$ About $70 \%$ of the patients with a new episode of SP show incomplete recovery within six weeks, 50\% report persistent complaints after six months, and $40 \%$ are not recovered after one year. ${ }^{4}$ Troublesome pain is the most prominent symptom in adult patients with SP until the age of 65 years. ${ }^{5}$ Prolonged and recurrent pain episodes result in frequent consultations. ${ }^{6}$ Within one year, $40 \%$ of the patients with SP have at least one re-consultation with the GP. ${ }^{4}$ Roughly $30 \%$ of the patients with SP report limitations in daily life and sick leave is common. ${ }^{3}$ It is suggested that prolonged and recurrent episodes generate substantial costs for care and sick leave. ${ }^{7}$ In general, indirect costs, such as costs caused by sick leave from paid work, are substantial and represent a higher burden to the economy than direct costs. ${ }^{8}$ Hence, there is a great need to improve diagnosis and prognosis from both the individual patient perspective as well as from a societal perspective.

Subacromial disorders are considered to be the most common pathology affecting the shoulder. In $80 \%$ of the cases with SP in primary care, the rotator cuff is the prominently affected anatomical structure. ${ }^{4}$ The spectrum of subacromial pathology is extensive and includes rotator cuff tendinopathy (tendinosis), calcific tendinitis, partialor full-thickness tears, and acute or chronic subacromial-subdeltoid bursitis. ${ }^{9-11}$ In primary care, the prevalence of these disorders are unknown. Studies in secondary care have shown a prevalence ranging from $30-39 \%$ for tendinopathy, $13-15 \%$ for calcific tendinitis, $13-51 \%$ for partial-thickness tears, $24-70 \%$ for full-thickness tears, and $12-56 \%$ for bursitis. ${ }^{12}$ Conflicting theories have been proposed to explain the mechanisms leading to pathology, and more research is needed to understand the disease process. ${ }^{10,13,14}$ However, the dominant view is that many shoulder complaints have their origin in a dynamic pathology, with subacromial impingement as the initial stage, and rotator cuff tears as the final stage. ${ }^{15,16}$

For all these specific subacromial disorders, specific therapies are available. ${ }^{9,17}$ After the mainstay of paracetamol or non-steroidal anti-inflammatory drugs (NSAIDs), tendinopathy preferably is treated with physiotherapy, calcific tendinitis and bursitis with subacromial corticosteroid injections, partial-thickness tears with physiotherapy, and in case of full-thickness tears surgery should be considered. Unfortunately, physical examinations used to evaluate the various disorders are fraught with uncertainty. ${ }^{4,10,18}$ As a result, the diagnostic phase does not often lead to a patho-anatomical diagnosis. Therefore, the guideline for SP of the Dutch College of General Practitioners (DCGP) advises GPs to start treatment based on patients' signs and symptoms rather than on a 
suspected patho-anatomical diagnosis. The advised treatment for all patients consists of a stepwise approach, which starts with advice and paracetamol or NSAIDs for 2 weeks. In persisting cases subacromial corticosteroid injections and referral to a physiotherapist are advised, depending on the level of pain and functional limitations respectively. Referral to a specialist or imaging modalities are advised if these usual care treatments fail. ${ }^{4}$

The current diagnostic strategy leads to a substantial case mix, and the stepwise treatment approach is expected to dilute the effects of the indicated interventions in the total population considerably. This approach delays specific therapy tailored to the pathology, which is remarkable knowing that a more effective approach to SP is available, which can lead to a better prognosis and less costs. We hypothesise that the current stepwise approach can be improved by giving the GP more evidence as to the patho-anatomical origin of the symptoms of SP. Due to the sufficient diagnostic accuracy, ${ }^{12,19,20}$ and clear relationship between US diagnosis and available evidence based therapies, applying US in the management of SP in primary care can solve this problem. US has the advantage of being non-invasive, relatively inexpensive, and producing high-resolution dynamic images of the shoulder. However, additional costs of management with US in patients with SP should be balanced by an increase in patients' health status and/or monetary savings for society. This cost-effectiveness, which has not been studied before, is the subject of our trial. This study foresees in the evidence gap that is addressed in the SP guideline of the DCGP.

Therefore, the primary objective of the present study is to investigate the effects of diagnostic US and its related tailored treatment decisions on clinical recovery and costs (cost-effectiveness) compared to usual care in individuals with non-chronic SP (pain less than 3 months) in primary care. The secondary objectives of the study are: (i) to investigate the effects of diagnostic US and its related treatment decisions on shoulder pain, performance of daily activities, and health-related quality of life compared to the usual care; (ii) to determine the prevalence of the specific subacromial disorders based on US reports; (iii) to evaluate to what extent the introduction of US influences management decisions, what type of treatment is provided to what type of patient (patient characteristics), and the adherence to the initially applied treatment.

\section{Methods/design}

\section{Design}

In a pragmatic design, which initially follows the guideline for SP of the DCGP, ${ }^{4}$ a study consisting of two phases will be carried out: a Qualification Period of two weeks 
followed by a randomised controlled trial (RCT) with a 50 week follow-up period (Figure 5.1). The Qualification period aims to filter out patients with a favorable natural course. During the 2-week Qualification period all patients are advised to start with paracetamol or NSAIDs in maximum dosage on a time contingent base, receive advice regarding activities of daily living, work, hobbies and sports. This advice fits within the first line treatment as recommended in the guideline for SP of the DCGP. Moreover, patients are referred for US of the shoulder to the radiology department of the Maastricht University Medical Centre (MUMC) or Orbis Medical Centre (OMC) in Sittard-Geleen, The Netherlands. Based on the qualification assessment at 2 weeks, patients with insufficient improvement qualify for the RCT.

These patients are randomly assigned to the intervention or the control group. The therapies used in both groups are the same except that therapies used in the intervention group will be tailored based on the US results. Primary and secondary outcome measures will be assessed at baseline, 13, 26, 39 and 52 weeks after inclusion. Patient recruitment started in November 2010 and patients will be included until October 2012. The Medical Ethics Committee of the Maastricht University Medical Centre has approved this protocol (NTR2403). This trial is officially called the Maastricht Ultrasound Shoulder pain Trial (MUST).

\section{Setting}

Patients for this trial will be recruited and treated by 21 GPs, working in 11 general practices, in the Westelijke Mijnstreek, a region in the southern part of the Netherlands. A total of 80 general practitioners received a letter inviting them to participate in this study. Of them, 21 GPs agreed to participate in the study. They attended a 2-hour instruction workshop with their practice assistants. This workshop provided information about the guideline for SP of the DCGP, the inclusion and randomisation procedures, as well as the interventions to be applied. All participating GPs were asked to give the names of their preferred physiotherapy practices. These physiotherapists were additionally invited for the workshop. In total 26 physiotherapists from 12 of the 14 invited physiotherapy practices attended the workshop. The physiotherapists were presented with an evidence based statement regarding subacromial disorders in a separate 1-hour parallel program. ${ }^{21}$ Those two practices not represented, received a handout of the presentation and study materials. 


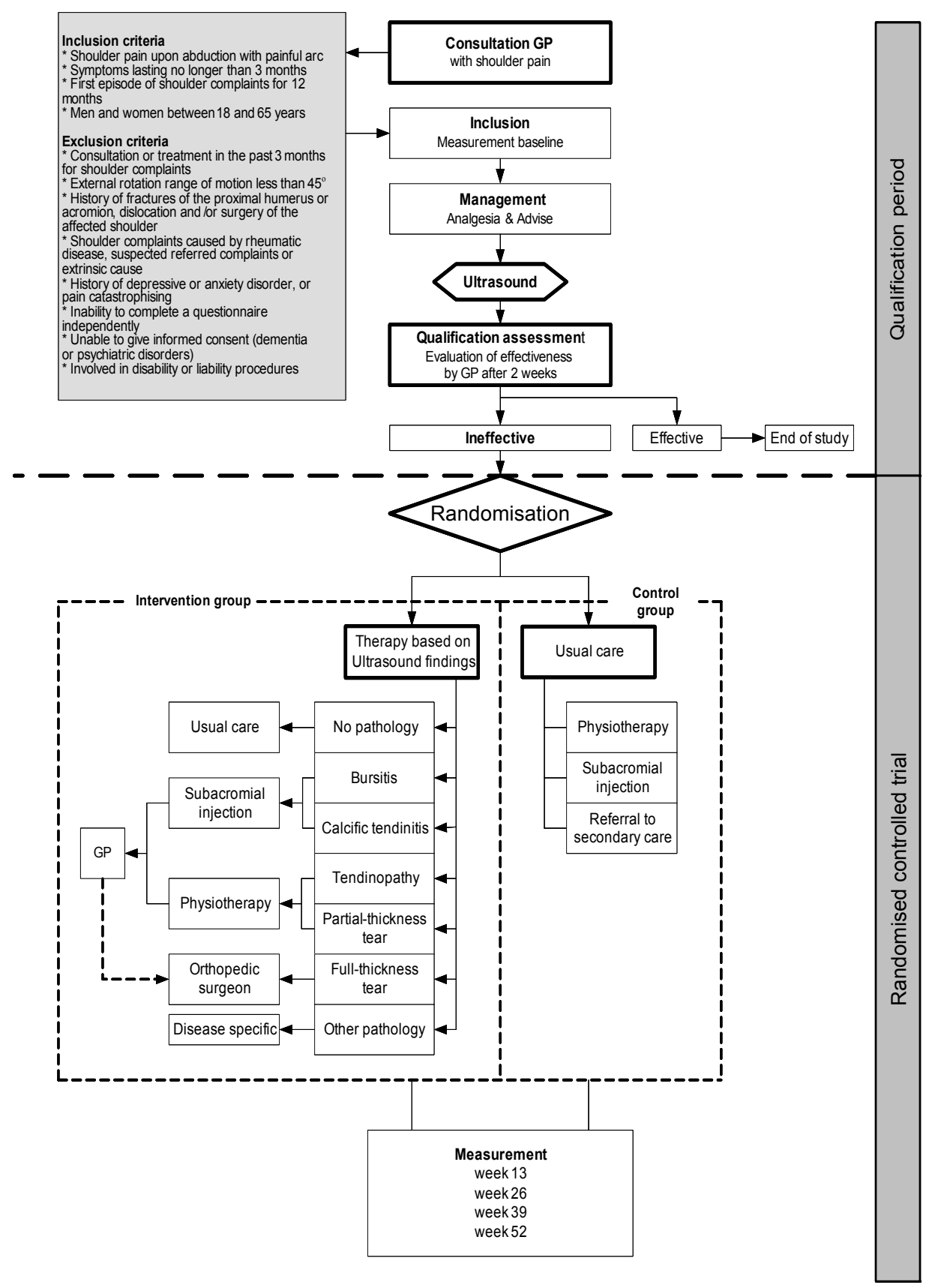

Figure 5.1 Flowchart of the study 


\section{Study population}

The study population will comprise of patients with SP, who are physically active with troublesome pain, and visit their GP with an episode of SP. To be eligible for recruitment patients have to fulfil the following eligibility criteria: (i) shoulder pain upon abduction with painful arc; (ii) symptoms lasting no longer than three months; (iii) first episode of SP for 12 months; (iv) age between 18 and 65 years. Exclusion criteria will be: (i) consultation or treatment for SP in the past three months; (ii) glenohumeral external rotation range of motion less than 45 degrees as this is a reason to suspect a glenohumeral disorder like osteoarthritis or a frozen shoulder; (iii) history of fractures of the proximal humerus or acromion, dislocation and/or surgery of the affected shoulder; (iv) shoulder complaints caused by rheumatic disease, suspected referred complaints or extrinsic cause; ( $v$ ) history of depressive or anxiety disorders, or pain catastrophising; (vi) inability to complete a questionnaire independently; (vii) unable to give informed consent (dementia or psychiatric disorders); (viii) involved in disability or liability procedures.

\section{Interventions}

Before randomisation, US of the shoulder is performed by a radiologist with 8 to 20 years of experience in musculoskeletal US at the MUMC or OMC using a protocolbased scanning approach (Table 5.1). ${ }^{22-26}$ US is an accurate diagnostic instrument to diagnose subacromial disorders. ${ }^{12}$ The distinguishable disorders are tendinopathy, calcific tendinitis, partial and full thickness tears, and subacromial bursitis.

Table 5.1 Ultrasound examination technique and diagnosis ${ }^{22-26}$

\begin{tabular}{|c|c|}
\hline \multicolumn{2}{|l|}{ Positioning of the patient } \\
\hline \multicolumn{2}{|c|}{$\begin{array}{l}\text { The patient will be seated on a rotating stool. The elbow will be positioned in } 90^{\circ} \text { flexion with the hand } \\
\text { unsupported in supination (neutral position). The patient's arm will be placed in a series of positions to allow } \\
\text { optimal assessment of all structures. }\end{array}$} \\
\hline \multicolumn{2}{|l|}{ Structures and scan position } \\
\hline \multicolumn{2}{|c|}{$\begin{array}{l}\text { The examination includes evaluation of the biceps, subscapularis, supraspinatus, infraspinatus, and teres } \\
\text { minor tendon; the acromioclavicular (AC) joint; the subacromial-subdeltoid bursa; and posterior labrum. } \\
\text { Each tendon will be assessed by means of scanning planes oriented according to their long- and short-axis, } \\
\text { and from their myotendinous junction to the bony insertion. Hyperemia/neovascularity will be } \\
\text { semiquantitatively assessed and divided into four grades (0-3) with color doppler. }\end{array}$} \\
\hline Long head of the biceps tendon & Arm in neutral position. \\
\hline Subscapularis tendon & Arm in neutral position and during external rotation. \\
\hline Supraspinatus tendon & $\begin{array}{l}\text { Arm behind patient's back, with the hand close to the opposite scapular } \\
\text { tip (Crass position). If this position is not possible patient's hand palm } \\
\text { will be placed on the superior aspect of the iliac wing with the elbow } \\
\text { flexed, directed posteriorly and toward the midline (Modified Crass or } \\
\text { Middleton position). }\end{array}$ \\
\hline
\end{tabular}


Table 5.1 (continued)

\begin{tabular}{|c|c|}
\hline Infraspinatus tendon & $\begin{array}{l}\text { Forearm in supination on the ipsilateral thigh or across the front of } \\
\text { patient's chest, with the hand resting on the opposite shoulder. } \\
\text { Visualization will be enhanced by passive internal and external } \\
\text { rotation, during real-time dynamic imaging. }\end{array}$ \\
\hline Subacromial bursa & $\begin{array}{l}\text { Arm in neutral position, also with the patient's arm behind his or her } \\
\text { back and during testing for subacromial impingement. }\end{array}$ \\
\hline AC-joint & $\begin{array}{l}\text { Arm in neutral position with the transducer in a coronal plane, also } \\
\text { shifting and rotating the transducer over the acromion. }\end{array}$ \\
\hline Subacromial impingement & $\begin{array}{l}\text { The transducer will be placed coronally with its medial margin at the } \\
\text { lateral margin of the acromion. The patient will abduct his arm while } \\
\text { it is in internal rotation. The supraspinatus tendon and bursa } \\
\text { should glide easily under the acromion until the greater tuberosity } \\
\text { nearly touches it. Next, this procedure will also be performed during } \\
\text { flexion, with the transducer placed sagittally with its posterior } \\
\text { margin at the anterior margin of the acromion. }\end{array}$ \\
\hline \multicolumn{2}{|l|}{ Criteria for diagnosing pathology } \\
\hline \multicolumn{2}{|c|}{$\begin{array}{l}\text { In general, secondary ultrasound signs, such as greater tuberosity cortical irregularity, joint and/or bursal } \\
\text { fluid, are valuable in the diagnosis of rotator cuff tears. All ultrasound examinations are interpreted } \\
\text { prospectively and digital stored. The length and width or degree of retraction of a tear, when present, will be } \\
\text { measured. }\end{array}$} \\
\hline Tendinopathy & $\begin{array}{l}\text { The tendon is locally or diffusely swollen and has a heterogeneous } \\
\text { hypoechoic appearance. }\end{array}$ \\
\hline Calcific tendonitis & $\begin{array}{l}\text { The tendon contains hyperechoic foci. Three main types of calcium } \\
\text { deposits can be identified: hyperechoic foci with a well-defined } \\
\text { acoustic shadow (Type I); hyperechoic foci with a faint (Type II) or } \\
\text { absent shadow (Type III). Dynamic examination to reveal } \\
\text { impingement and local hyperemia will be assessed }\end{array}$ \\
\hline Full-thickness rotator cuff tear & $\begin{array}{l}\text { Any of the following criteria is present: focal thinning of the tendon, } \\
\text { complete non-visualization of the tendon, focal discontinuity in the } \\
\text { homogeneous echogenicity of the tendon without focal thinning, or } \\
\text { inversion of the superficial bursa contour and/or hyperechoic } \\
\text { material in the location of the tendon that fails to move with the } \\
\text { humeral head during real-time dynamic imaging. }\end{array}$ \\
\hline Partial-thickness rotator cuff tear & $\begin{array}{l}\text { Any of the following criteria is present: a hypoechoic discontinuity in } \\
\text { the tendon in which the lesion involves either the bursal or articular } \\
\text { side of the tendon, or a mixed hyperechoic and hypoechoic region } \\
\text { within the tendon. The area does not change its appearance on short- } \\
\text { and long-axis scans and while tilting the transducer over the tendon. }\end{array}$ \\
\hline $\begin{array}{l}\text { Biceps tendon tear } \\
\text { (partial or complete) }\end{array}$ & $\begin{array}{l}\text { The tendon contains respectively an anechoic cleft and fluid in the } \\
\text { sheath or a complete anechoic cleft through the tendon or a fluid } \\
\text { filled sheath separating the retracted ends of the torn tendon. }\end{array}$ \\
\hline
\end{tabular}


Table 5.1 (continued)

\begin{tabular}{|c|c|}
\hline Subacromial bursitis & $\begin{array}{l}\text { A thin hypoechoic layer of more than } 2 \mathrm{~mm} \text { sandwiched between } \\
\text { hyperechoic peribursal fat, in turn sandwiched between the } \\
\text { hypoechoic deltoid muscle and supraspinatus tendon with } \\
\text { hyperemia and/or a teardrop-shaped structure with the most } \\
\text { distended segment of the bursa most distal and most dependent. } \\
\text { Fluid in the bursa has also to be considered as a secondary sign of a } \\
\text { partial-thickness rotator cuff tear at the bursal side or a full- } \\
\text { thickness tear. }\end{array}$ \\
\hline Subacromial impingement & $\begin{array}{l}\text { The supraspinatus tendon catches on or bunches up lateral or } \\
\text { anterior to the acromion. The presences of bursal thickening } \\
\text { without a history of inflammatory arthropathy and/or movement of } \\
\text { fluid into the most lateral portion of the bursa during abduction } \\
\text { are secondary signs of impingement. }\end{array}$ \\
\hline AC osteoarthritis & $\begin{array}{l}\text { Any of the following criteria is present: narrowing of the joint space, } \\
\text { irregularities and/or osteophytes of the articular bone surfaces, } \\
\text { and/or para-articular cysts. }\end{array}$ \\
\hline Os acromiale & $\begin{array}{l}\text { Well defined cortical discontinuity of the superior aspect of the } \\
\text { acromion, often mimicking a double AC-joint. }\end{array}$ \\
\hline
\end{tabular}

\section{Intervention group}

The advised evidence based, tailored treatment steps are described in Table 5.2. . $^{4,17,21,27-36}$ To prevent treatment of supposed asymptomatic pathology, GPs will link US pathology to history and findings from physical examination. In case pathology other than rotator cuff disorders is diagnosed, it will be treated according to this diagnosis (e.g. in cases of signs of rheumatoid arthritis patients are referred to a rheumatologist). If there is no detectable pathology, usual care according to the guideline for SP of the DCGP will be advised. ${ }^{4}$ In cases where multiple US findings are present, the most relevant abnormality will be selected by the GP on the basis of the clinical findings. With an explanation, and within the recommendations made in the guideline for SP of the DCGP, GPs are allowed to deviate from the advised treatments steps. The treatments are standardised. 
Table 5.2 Ultrasound diagnosis tailored treatment steps

\begin{tabular}{|c|c|c|}
\hline \multicolumn{3}{|c|}{ Tendinopathy } \\
\hline \multirow[t]{4}{*}{ Step 1} & \multicolumn{2}{|c|}{ Physical therapy protocol $^{21}$} \\
\hline & Content & $\begin{array}{l}\text { An evidence based exercise protocol. Initially, supervised exercises } \\
\text { With manual therapy is recommended. During that time, patients } \\
\text { should be instructed in a home program. } \\
\text { - Exercises: range of motion, flexibility, strengthening, scapular } \\
\text { stabilization } \\
\text { - Manual therapy: joint and soft tissue mobilization techniques } \\
\text { augment the effect of the exercise program }\end{array}$ \\
\hline & Duration & 6-12 weeks \\
\hline & Evaluation & In case of insufficient improvement, step 2 is advised \\
\hline \multirow[t]{2}{*}{ Step 2} & \multicolumn{2}{|c|}{ Re-consultation GP } \\
\hline & Content & $\begin{array}{l}\text { Treatment will be left to the discretion of the GP (possible options: } \\
\text { wait and- see policy; subacromial corticosteroid injection; referral to } \\
\text { an orthopedic surgeon) }\end{array}$ \\
\hline \multicolumn{3}{|c|}{ Calcific tendonitis } \\
\hline \multirow[t]{4}{*}{ Step 1} & \multicolumn{2}{|c|}{ Subacromial corticosteroid injection ${ }^{4,9,30}$} \\
\hline & Content & $\begin{array}{l}\text { Subacromial corticosteroid injection with a mixture of } 1 \mathrm{ml} \\
\text { triamcinoloni acetonidum } 40 \mathrm{mg} / \mathrm{ml} \text { and } 4-9 \mathrm{ml} \text { lidocaine } 10 \mathrm{mg} / \mathrm{ml}\end{array}$ \\
\hline & Procedure & Posterolateral entry approach to the subacromial region \\
\hline & Evaluation & $\begin{array}{l}\text { In case of insufficient improvement after } 2-4 \text { weeks, a second } \\
\text { injection can be given or step } 2 \text { is advised }\end{array}$ \\
\hline \multirow[t]{2}{*}{ Step 2} & \multicolumn{2}{|c|}{ Re-consultation GP } \\
\hline & Content & $\begin{array}{l}\text { Treatment will be left to the discretion of the GP (possible options: } \\
\text { wait-and-see policy; referral to a physiotherapist or an orthopedic } \\
\text { surgeon) }\end{array}$ \\
\hline \multicolumn{3}{|c|}{ Subacromial-subdeltoid bursitis } \\
\hline \multirow[t]{4}{*}{ Step 1} & \multicolumn{2}{|c|}{ Subacromial corticosteroid injection ${ }^{9,28,36}$} \\
\hline & Content & $\begin{array}{l}\text { Subacromial corticosteroid injection with a mixture of } 1 \mathrm{ml} \\
\text { triamcinoloni acetonidum } 40 \mathrm{mg} / \mathrm{ml} \text { and } 4-9 \mathrm{ml} \text { lidocaine } 10 \mathrm{mg} / \mathrm{ml}\end{array}$ \\
\hline & Procedure & Posterolateral entry approach to the subacromial region \\
\hline & Evaluation & $\begin{array}{l}\text { In case of insufficient improvement after } 2-4 \text { weeks, a second injection } \\
\text { can be given or step } 2 \text { is advised }\end{array}$ \\
\hline \multirow[t]{2}{*}{ Step 2} & \multicolumn{2}{|c|}{ Re-consultation GP } \\
\hline & Content & $\begin{array}{l}\text { Treatment will be left to the discretion of the GP (possible options: } \\
\text { wait-and-see policy; referral to a physiotherapist or an orthopedic } \\
\text { surgeon) }\end{array}$ \\
\hline \multicolumn{3}{|c|}{ Partial-thickness tear } \\
\hline \multirow[t]{5}{*}{ Step 1} & \multicolumn{2}{|c|}{ Physical therapy ${ }^{33,34}$} \\
\hline & Content & These patients are treated similarly to those with tendinopathy \\
\hline & Duration & 6-12 weeks \\
\hline & Evaluation & In case of insufficient improvement, step 2 is advised. \\
\hline & Exception & Acute tears are immediately discussed with an orthopedic surgeon \\
\hline \multirow[t]{2}{*}{ Step 2} & \multicolumn{2}{|c|}{ Re-consultation GP } \\
\hline & Content & $\begin{array}{l}\text { Treatment will be left to the discretion of the GP with the advice to } \\
\text { administer a subacromial corticosteroid injection or to consult } \\
\text { an orthopedic surgeon }\end{array}$ \\
\hline
\end{tabular}


Table 5.2 (continued)

\begin{tabular}{|l|l|l|}
\hline \multirow{2}{*}{ Full-thickness tear } \\
\hline \multirow{2}{*}{ tep 1} & Referral to an orthopedic surgeon ${ }^{9,17,27,29,32,35}$ \\
\cline { 2 - 3 } & Content & Treatment will be left to the discretion of the orthopedic surgeon \\
\hline \multirow{2}{*}{ Step 2} & Physical therapy (in case step 1 is not executed) \\
\cline { 2 - 3 } & Content & These patients are treated similarly to those with tendinopathy \\
\hline
\end{tabular}

\section{Control group}

Usual care according to the guideline for SP of the DCGP will be applied in the control group. It consists of a pragmatic, stepwise approach; a wait-and-see policy with advice and analgesia for another 2 weeks; in persisting cases corticosteroid injections and referral to a physiotherapist are advised, depending on the level of pain and functional limitations respectively; referral to a hospital specialist is advised if conservative treatment fails. ${ }^{4}$

\section{Randomisation and allocation}

Based on the qualification assessment at 2 weeks, unrecovered patients (measured by the Global Perceived Effect questionnaire; see below) qualify for the RCT and are randomly assigned by central block randomisation (blocks of 4 ) to the intervention or control group after stratification for age ( $\geq 50$ years). Neither the patient nor the GP can be blinded for the allocated treatment. However, US results are only disclosed to GPS of those patients in the intervention group, as well as those patients themselves. In case a patient is allocated to the intervention group, the GP receives the US result and the advised corresponding treatment strategy. In the control group, neither the patients nor the GPS receive the US results. Their US results will be presented to the GP at the end of patients' follow-up period.

The radiologist performing the US, is not allowed to communicate with the patients about the US findings and results. In case a fracture, septic bursitis or arthritis, or a lifethreatening disorder (e.g. tumour) is diagnosed, the radiologist will immediately inform the GP with c.c. to the investigator, and the patient will be excluded and not randomised.

\section{Outcome assessment}

At baseline, demographic information will be collected including age, sex and profession, as well as disease specific information regarding the affected side, onset, duration of symptoms, possible cause of complaints, history of shoulder complaints, 
neck complaints and dominant arm. A number of outcome measures will be collected at baseline, 13, 26, 39 and 52 weeks after inclusion (Table 5.3).

Table $5.3 \quad$ Outcome measures

\begin{tabular}{|c|c|c|c|c|c|}
\hline & $\mathrm{T}_{0}$ & $\mathrm{~T}_{1}$ & $\mathrm{~T}_{2}$ & $\mathrm{~T}_{3}$ & $\mathrm{~T}_{4}$ \\
\hline \multicolumn{6}{|l|}{ Primary outcome } \\
\hline Patient-perceived recovery (GPE) & & + & + & + & + \\
\hline \multicolumn{6}{|l|}{ Secondary outcomes } \\
\hline Performance of daily activities (SDQ) & + & + & + & & + \\
\hline Shoulder pain (SPS) & + & + & + & & + \\
\hline Quality of daily life (EQ-5D) & + & + & + & & + \\
\hline \multicolumn{6}{|l|}{ Other measures } \\
\hline Costs & & + & + & + & + \\
\hline $\begin{array}{l}\text { Number of re-consultations, corticosteroid injections, } \\
\text { diagnostic imaging procedures, referrals to } \\
\text { physiotherapy and hospital }\end{array}$ & & & & & + \\
\hline
\end{tabular}

$T_{0}$ - baseline; $T_{1}-13$ weeks; $T_{2}-26$ weeks; $T_{3}-39$ weeks; $T_{4}-52$ weeks; GPE - Global Perceived Effect; SDQ Shoulder Disability Questionnaire; SPS - Shoulder Pain Score; EQ-5D - Euroqol five-item quality of life questionnaire

\section{Primary outcome measure}

The primary outcome measure for the clinical effectiveness is the patient-perceived recovery using the Global Perceived Effect questionnaire (GPE); a one-item score concerning recovery following treatment, measured on a seven-point ordinal scale. ${ }^{37}$ Patients are considered to be recovered when they report to be much improved or fully recovered. Together with disease-specific functional status measures, this is considered to be an important outcome variable for shoulder complaints.

\section{Secondary outcome measures}

\section{Shoulder Pain Score (SPS)}

The SPS is a questionnaire to assess pain experienced by patients with shoulder disorders and includes a 24-hour recall frame. The score consists of six pain symptom questions and a 10-point Scale. ${ }^{38}$ The SPS has been proved to be a useful instrument for following the course of the disorder over time, and gives an indication when a patient feels cured. Each question receives a maximum of four points. The VAS is also transposed to a four-point scale $(0=1,1-3=2,4-6=3,7-10=4)$. The minimum SPS score is seven points, the maximum score 28 . 


\section{The Shoulder Disability Questionnaire (SDQ)}

The SDQ assesses the performance of daily activities. This variable will be assessed by a 16-item questionnaire for functional status limitation in patients with shoulder disorders and assesses the past 24 hours. $^{39}$ The 16 questions can be answered with either yes, no or not applicable. The final SDQ-score will be calculated by dividing the number of positive responses by the total number of applicable items, and multiplying this score by 100 . Consequently, the SDQ-score can range from 0 to 100 with a higher score indicating more severe disability.

\section{The Euroqol five-item quality of life questionnaire (EQ-5D)}

The EQ-5D is one of the most commonly used generic (that is not disease specific) measures used to quantify the health related quality of life in people with musculoskeletal disorders. ${ }^{40,41}$ It is a patient-reported measure that consists of two sections. The first section comprises five questions with three levels of severity in each (1=no problem, $2=$ moderate problem, $3=$ =severe problem) that covers five dimensions of health: mobility, self-care, usual activities, complaints/discomfort, and anxiety/ depression. This generates 243 theoretically possible health states. Calculation of the index score will be performed according to the European recommendations. ${ }^{42}$ The second section is a visual analogue scale ranging from 0 (worst imaginable health state) to 100 (best imaginable health state).

\section{Costs}

Intervention costs, direct and non-health care costs, as well as indirect costs will be collected. A questionnaire composed of 24 questions regarding resource use and expenses in the last three months will be used. In addition, the research team will contact the GPs, physiotherapists, and hospital specialists in case patients have been referred, for treatment costs. ${ }^{43}$ Standard unit cost data will be derived from reliable published sources. ${ }^{44}$ The costs related to the intervention itself (application of US) will be assessed by detailed cost pricing.

\section{Other assessments}

Also in addition, it will be evaluated to what extent patients are blinded for the US findings and results prior to randomization, to what extent the introduction of US influences management decisions, what type of treatment is provided to what type of patient (patient characteristics), and what adherence rates to the initial applied treatment can be shown. 


\section{Sample size}

The sample size calculation is based on a recovery rate (measured by GPE) of $60 \%$ in the control group ${ }^{4}$ and $80 \%$ in the intervention group after 52 weeks, a two sided-alpha of 0.05 , a statistical power of 0.80 , and a drop-out rate at 52 weeks of $10 \%{ }^{45,46}$ We need 90 patients per study group to detect the difference of $20 \%$ between the study groups after 52 weeks; the minimal clinical important difference. With the expectation that the Qualification period filters out $20 \%$ of the patients, we need to include 226 patients. With up to 100 new patient encounters per GP per year ${ }^{1-4}$ and approximately 25 eligible patients per GP per year, two years of recruitment, $50 \%$ consent to the study, and 50\% drop-out for other reasons, we need 20 GPs to participate to include the required 226 patients. Based on the reported prevalence, and our experience, we expect to encounter enough patients with symptomatic US pathology to complete recruitment within two years.

\section{Data analysis}

The primary analysis will be intention-to-treat and will compare the patient-perceived recovery measured by the Global Perceived Effect questionnaire (GPE) at 52 weeks after randomisation of patients managed by US tailored treatment (intervention group) and those having received care as usual (control group). In order to study the influence of protocol violations on the study outcomes, a per protocol analysis will be performed. Patients with documented deviations from the study protocol (that is no adherence to the treatment steps mentioned in Table 2) will be excluded from this analysis.

Continuous variables will be presented as mean \pm standard deviation and categorical variables as number (\%). The longitudinal trend of primary and secondary parameters will be compared between the intervention and control group using logistic and linear mixed models to take into account the dependency of repeated measurements and nesting structure of data (patients within GP practices). Baseline characteristics that a priori are considered to be possible prognostic factors (fast or gradual development of SP, possible cause of SP, dominant shoulder affected, concomitant neck complaints, and physical work with upper extremities), will be included in the mixed models.

An economic evaluation will be performed from both a health care and societal perspective with a time horizon of 52 weeks. The incremental cost-effectiveness ratios will be expressed as the costs per additionally recovered patient (from a health care perspective) and the costs per Quality Adjusted Life Years (from a societal perspective). Sensitivity analyses will be performed to assess the influence of relevant factors. Finally, bootstrap analysis will be performed to quantify the uncertainty surrounding the incremental costs and effects. Based on these results, a Cost-Effectiveness 
Acceptability Curve will be constructed to show the probability that the intervention is cost-effective.

\section{Discussion}

This study uses a randomised controlled trial design to investigate whether US as a diagnostic triage tool is more effective on clinical recovery and is more cost-effective than continuation of usual care in patients with non-chronic SP in general practice. This study foresees in the evidence gap that is addressed in the SP guideline of the DCGP. Primary inclusion criterion is the GP's suspicion of a subacromial disorder being the primary cause of SP. GPs are daily facing patients with SP and, based on the guideline for SP of the DCGP, they are able to differentiate between subacromial, glenohumeral and extrinsic disorders for SP. The pragmatic approach of this trial leaves them very close to daily practice when considering recruiting patients for this study.

This study is designed as a randomised controlled trial. However, since blinding patients is not possible in this pragmatic study, information bias has to be taken into account. As a stratagem to prevent this, radiologists are not allowed to discuss the US findings and results with patients. To evaluate this information bias, we included a question in the questionnaire at 13 weeks. Patients allocated to the control group and their GPs do not receive the US result until the end of patients' follow-up period.

The treatment options for the intervention and control group do not differ significantly. However, the significant difference between the treatment regimes in the intervention and control groups is that patients in the intervention group receive tailored treatment according to their patho-anatomical disease state. This in contrast with patients in the control group, who are treated according to the guideline SP of the DCGP, and receive treatment based on complaints. In this cost-effectiveness study we compare diagnosis treatment combinations, and not only treatments. It is our hypothesis that these tailored treatment regimes, applying evidence based treatment without delay, have a positive effect on patient recovery and costs. Moreover, the prevention of unnecessary interventions can contribute to this effect.

In general, the accuracy of US is operator dependent. In experience hands US is an accurate diagnostic instrument to diagnose subacromial disorders. ${ }^{12}$ For study purposes one can chose for a single operator or multiple operators per radiology department. For two reasons we choose for multiple operators. First, due to our design (the US has to be made within two weeks after patient inclusion, and the inclusion period runs for two years), the radiology departments were not able to guarantee a single operator. Second, a single operator would limit the external validity, as in The Netherlands it is 
common that within a radiology department, more radiologists are experienced in musculoskeletal ultrasound. Therefore, all US in our study are only made by experienced radiologists in musculoskeletal ultrasound. For study purposes, these radiologists use a standardized protocol (Table 5.1), which was developed by the project group and radiology departments.

It is likely that degeneration is one of the mechanisms leading to rotator cuff pathology. ${ }^{14}$ As degeneration is part of the normal aging process, and therefore a potential confounder, we choose to stratify for age. In this respect, we realise that degeneration is a continuum, and evidence for a clear cut-off point for age as a confounder is lacking. Although arbitrary, we think it is plausible to set this cutt-off point for age at 50 years.

Rotator cuff tears are also common in asymptomatic and unselected populations. ${ }^{47,48}$ The incidence of tears is known to increase with age and can be considered as part of the normal ageing process. ${ }^{47}$ In this respect, we realise that overdiagnosis is to be avoided. However, the current diagnostic strategy leads to underdiagnosis. We do realise that in general a certain percentage of US recorded pathology is not directly responsible for the reported symptoms. Therefore, to prevent treatment of asymptomatic pathology, US pathology will be linked to medical history and physical examination.

As the prevelances of the different subacromial disorders in primary care are unknown, it is questionable whether we will encounter enough patients to start tailored treatment in the intervention group. Based on the reported prevalences in secondary care, and our experiences with patients in primary care who underwent shoulder US, we expect to encounter enough patients with different symptomatic US pathologies to start tailored treatment.

Physiotherapists generally utilise a variety of techniques such as mobilization, soft tissue massage, exercises and education. Often, their program is based on their own experiences. To ensure a consistent program, we trained the physiotherapists in an evidence based approach for subacromial disorders (released by the Royal Dutch Society for Physical Therapy). ${ }^{21}$

This study will ensure that the cost-effectiveness of US as a diagnostic triage tool in primary care is adequately evaluated. It will fill the gaps in the current evidence base and may guide clinical practice and policy. Importantly, the results obtained can be used to formulate (new) guidelines. In the Netherlands, guidelines of the DCGP, including the one for SP, are seen as the "gold standard" for the management of frequently occurring diseases and health problems. If US is considered cost-effective, this pragmatic study will contribute to dissemination. This potentially reduces unnecessary interventions, re-consultations, and referrals to physiotherapists and 
hospital. Referrals will be based on more adequate questions. If this is a negative trial, then resources will be saved by not promoting US in the early management of SP. Overall, quality of care in patients with SP will improve. 


\section{References}

1. Bot SD, van der Waal JM, Terwee CB, et al. Incidence and prevalence of complaints of the neck and upper extremity in general practice. Ann Rheum Dis. 2005;64:118-123.

2. Feleus A, Bierma-Zeinstra SM, Miedema HS, Bernsen RM, Verhaar JA, Koes BW. Incidence of nontraumatic complaints of arm, neck and shoulder in general practice. Man Ther. 2008;13:426-433.

3. Picavet HS, Schouten JS. Musculoskeletal pain in the Netherlands: prevalences, consequences and risk groups, the DMC(3)-study. Pain. 2003;102:167-178.

4. Winters JC, van der Windt DAWM, Spinnewijn WEM, et al. Shoulder pain guideline of the Dutch College of General Practitioners (in Dutch). Huisarts Wet. 2008;51:555-565.

5. Parsons S, Breen A, Foster NE, et al. Prevalence and comparative troublesomeness by age of musculoskeletal pain in different body locations. Fam Pract. 2007;24:308-316.

6. Breivik H, Collett B, Ventafridda V, Cohen R, Gallacher D. Survey of chronic pain in Europe: prevalence, impact on daily life, and treatment. Eur J Pain. 2006;10:287-333.

7. Kuijpers T, van Tulder MW, van der Heijden GJMG, Bouter LM, van der Windt DIAWM. Costs of shoulder pain in primary care consulters: a prospective cohort study in The Netherlands. BMC Musculoskelet Disord. 2006;7:83.

8. Jensen MK, Sjogren P, Ekholm O, Rasmussen NK, Eriksen J. Identifying a long-term/chronic, non-cancer pain population using a one-dimensional verbal pain rating scale: an epidemiological study. Eur J Pain. 2004;8:145-152.

9. Baring T, Emery RJH, Reilly P. Management of rotator cuff disease: specific treatment for specific disorders. Best Pract Res Clin Rheumatol. 2007;21:279-294.

10. Lewis J, Tennent D, eds. How effective are diagnostic tests for the assessment of rotator cuff disease of the shoulder? London: Blackwell, BMJ Books; 2007. MacAuley D, Best TM, eds. Evidence based sports medicine.

11. Riley G. Tendinopathy - from basic science to treatment. Nat Clin Pract Rheumatol. 2008;4:82-89.

12. Ottenheijm RPG, Jansen MJ, Staal JB, et al. Accuracy of diagnostic ultrasound in patients with suspected subacromial disorders: a systematic review and meta-analysis. Arch Phys Med Rehabil. 2010;91: 1616-1625.

13. Cook JL, Purdam CR. Is tendon pathology a continuum? A pathology model to explain the clinical presentation of load-induced tendinopathy. Br J Sports Med. 2009;43:409-416.

14. Lewis JS, Raza SA, Pilcher J, Heron C, Poloniecki JD. The prevalence of neovascularity in patients clinically diagnosed with rotator cuff tendinopathy. BMC Musculoskelet Disord. 2009;10:163.

15. Bigliani LU, Levine WN. Subacromial impingement syndrome. J Bone Joint Surg Am. 1997;79:1854-1868.

16. Neer CSn. Impingment lesions. Clin Orthop Rel Res. 1983;173:70-77.

17. Grant HJ, Arthur A, Pichora DR. Evaluation of interventions for rotator cuff pathology: a systematic review. J Hand Ther. 2004;17:274-299.

18. Kelly SM, Brittle N, Allen GM. The value of physical tests for subacromial impingement syndrome: a study of diagnostic accuracy. Clin Rehabil. 2010;24:149-158.

19. de Jesus JO, Parker L, Frangos AJ, Nazarian LN. Accuracy of MRI, MR arthrography, and ultrasound in the diagnosis of rotator cuff tears: a meta-analysis. Am J Roentgenol. 2009;192:1701-1707.

20. Dinnes J, Loveman E, Mclntyre L, Waugh N. The effectiveness of diagnostic tests for the assessment of shoulder pain due to soft tissue disorders: a systematic review. Health Technol Assess. 2003;7:1-166.

21. Royal Dutch Society for Physical Therapy. Diagnosis and treatment of subacromial disorders (in Dutch). 2010. Available at: www.kngfrichtlijnen.nl

22. Bianchi S, Martinoli C. Ultrasound of the musculoskeletal system. Berlin Heidelberg New York: Springer; 2007.

23. Mack LA, Nyberg DA, Matsen FA, 3rd. Sonographic evaluation of the rotator cuff. Radiol Clin North Am. 1988;26:161-177.

24. Middleton WD, Reinus WR, Totty WG, Melson CL, Murphy WA. Ultrasonographic evaluation of the rotator cuff and biceps tendon. J Bone Joint Surg Am. 1986;68:440-450. 
25. van Holsbeeck M, Strouse PJ. Sonography of the shoulder: evaluation of the subacromial-subdeltoid bursa. Am J Roentgenol. 1993;160:561-564.

26. van Holsbeeck MT, Kolowich PA, Eyler WR, et al. US depiction of partial-thickness tear of the rotator cuff. Radiology. 1995;197:443-446.

27. Ainsworth R, Lewis JS. Exercise therapy for the conservative management of full thickness tears of the rotator cuff: a systematic review. Br J Sports Med. 2007;41:200-210.

28. Buchbinder R, Green S, Youd JM. Corticosteroid injections for shoulder pain. Cochrane Database Syst Rev. 2003(1):CD004016.

29. Ejnisman B, Andreoli CV, Soares BG, et al. Interventions for tears of the rotator cuff in adults. Cochrane Database Syst Rev. 2004(1):CD002758.

30. Hurt G, Baker CL, Jr. Calcific tendinitis of the shoulder. Orthop Clin North Am. 2003;34:567-575.

31. Kuhn JE. Exercise in the treatment of rotator cuff impingement: a systematic review and a synthesized evidence-based rehabilitation protocol. J Shoulder Elbow Surg. 2009;18:138-160.

32. Lahteenmaki HE, Hiltunen A, Virolainen P, Nelimarkka O. Repair of full-thickness rotator cuff tears is recommended regardless of tear size and age: a retrospective study of 218 patients. J Shoulder Elbow Surg. 2007;16:586-590.

33. Mantone J. Nonoperative treatment of rotator cuff tears. Orthop Clin North Am. 2000;31(2):295-311.

34. Matava MJ, Purcell DB, Rudzki JR. Partial-thickness rotator cuff tears. Am J Sports Med. 2005;33: 1405-1417.

35. Oh LS, Wolf BR, Hall MP, Levy BA, Marx RG. Indications for rotator cuff repair: a systematic review. Clin Orthop Rel Res. 2007;455:52-63.

36. Yu CM, Chen CH, Liu HT, Dai MH, Wang IC, Wang KC. Subacromial injections of corticosteroids and xylocaine for painful subacromial impingement syndrome. Chang Gung Med J. 2006;29:474-479.

37. Kamper SJ, Ostelo RW, Knol DL, Maher CG, de Vet HC, M.J. H. Global Perceived Effect scales provided reliable assessments of health transition in people with musculoskeletal disorders, but ratings are strongly influenced by current status. J Clin Epidemiol. 2010;63:760-766.

38. Winters JC, Sobel JS, Groenier KH, Arendzen JH, Meyboom-De Jong B. A shoulder pain score: a comprehensive questionnaire for assessing pain in patients with shoulder complaints. Scand J Rehabil Med. 1996;28:163-167.

39. de Winter AF, van der Heijden GJ, Scholten RJ, van der Windt DA, Bouter LM. The Shoulder Disability Questionnaire differentiated well between high and low disability levels in patients in primary care, in a cross-sectional study. J Clin Epidemiol. 2007;60:1156-1163.

40. Brooks R. EuroQol: the current state of play. Health Policy. 1996;37:53-72.

41. Picavet HS, Hoeymans N. Health related quality of life in multiple musculoskeletal diseases: SF-36 and EQ-5D in the DMC3 study. Ann Rheum Dis. 2004;63:723-729.

42. Greiner W, Weijnen T, Nieuwenhuizen $M$, et al. A single European currency for EQ-5D health states. Results from a six-country study. Eur J Health Econ. 2003;4:222-231.

43. van den Brink $M$, van den Hout WB, Stiggelbout AM, van de Velde CJ, Kievit J. Cost measurement in economic evaluations of health care: whom to ask? Med Care. 2004;42:740-746.

44. Oostenbrink JB, Koopmanschap MA, Rutten FFG. Dutch Cost Manual (in Dutch). Amstelveen: College voor Zorgverzekeringen; 2004.

45. Bergman GJ, Winters JC, Groenier KH, et al. Manipulative therapy in addition to usual medical care for patients with shoulder dysfunction and pain: a randomized, controlled trial. Ann Intern Med. 2004;141:432-439.

46. Geraets JJ, Goossens ME, de Bruijn CP, et al. Cost-effectiveness of a graded exercise therapy program for patients with chronic shoulder complaints. Int J Technol Assess Health Care. 2006;22:76-83.

47. Milgrom C, Schaffler M, Gilbert S, van Holsbeeck M. Rotator-cuff changes in asymptomatic adults. The effect of age, hand dominance and gender. J Bone Joint Surg. 1995;77:296-298.

48. Reilly P, Macleod I, Macfarlane R, Windley J, Emery RJH. Dead men and radiologists don't lie: a review of cadaveric and radiological studies of rotator cuff tear prevalence. Ann R Coll Surg Engl. 2006;88: 116-121. 


\section{Chapter}

Ultrasound imaging for tailored treatment of patients

with acute shoulder pain

Ramon PG Ottenheijm, Jochen WL Cals, René E Weijers, Kurt Vanderdood, Rob A de Bie, Geert-Jan Dinant Ann Fam Med 2015;13:53-55 


\section{Abstract}

The objective of this study was to assess the frequencies of ultrasound findings in patients with acute rotator cuff disorder in family medicine. In a prospective observational study, 129 patients aged 18-65 years with acute shoulder pain in whom the family physician suspected rotator cuff disease underwent ultrasound imaging. Rotator cuff disease was present in $81 \%$ of the patients, and $50 \%$ of them had multiple disorders. Calcific tendonitis was the most frequently diagnosed specific disorder. An age of 40 years or older was most strongly related to rotator cuff disease. Ultrasound imaging enables family physicians to rationalize treatment in nearly all patients who are aged 40 years and older with acute shoulder pain. 


\section{Introduction}

Although most patients with acute shoulder pain are cared for in family medicine, ${ }^{1}$ physical examinations used to evaluate the extensive spectrum of rotator cuff disease are often inadequate. ${ }^{2}$ As a result, recommended treatment for all patients in family medicine consists of stepwise treatment, regardless of the specific underlying cause. ${ }^{3,4}$ The disadvantage of this management approach is that it may lead to unnecessary interventions and a delay in specific treatment, with subsequent prolonged recovery. Ultrasound imaging of the shoulder has become an accepted diagnostic modality for evaluating rotator cuff disease, ${ }^{5}$ which potentially allows for more tailored treatment based on diagnostic subgroups. Against this background, it seemed useful to prospectively determine the frequencies of the disorders from the spectrum of rotator cuff disease in patients with shoulder pain in a family medicine setting. The objectives of this study were to prospectively assess the frequencies of specific findings as diagnosed with ultrasound in patients with acute shoulder pain, and to identify possible predictors of shoulder pain.

\section{Methods}

The present study was part of the Maastricht Ultrasound Shoulder pain Trial (MUST) study, a randomized controlled trial among patients in family medicine with acute shoulder pain. The design of the MUST study has been presented in detail elsewhere. ${ }^{6}$ We included 129 patients with acute shoulder pain in whom the family physician suspected rotator cuff disease. Patients were eligible if they had (1) shoulder pain upon abduction with painful arc; (2) symptoms having lasted for fewer than three months; (3) had no other episodes of shoulder pain in the previous 12 months; (4) were aged between 18 and 65 years.

All ultrasound examinations were obtained in real time using a Phillips IU22 (Phillips Medical Systems, Bothell, USA) with a 5-12 MHz broadband linear-array transducer (Phillips L12-5). All examinations were performed by experienced musculoskeletal radiologists at the Orbis Medical Centre, The Netherlands, using a protocol-based scanning approach. Standardized ultrasound diagnostic criteria for pathology were used. ${ }^{6}$ Ultrasound examinations were prospectively evaluated for abnormalities of the rotator cuff, the long head of the biceps tendon, the subacromial-subdeltoid, and the acromioclavicular joint, as well as for impingement.

We calculated means and standard deviations for continuous variables and percentages for categorical variables. Linear and logistic regression analyses were performed to determine the association of age, sex, and occupation with rotator cuff disorders in 
univariate analysis, followed by multivariate analysis, and expressed as odds ratios (ORs). Age was dichotomized at the most discriminating 5-year age cut-off. $P<0.05$ was defined as statistically significant. Data were analyzed using IBM SPSS 21 (International Business Machines Corp).

\section{Results}

The mean age of the 129 included patients was 49.1 years (SD 10.6), and the dominant shoulder was affected in 54\% (70/129). Table 6.1 displays the frequencies of rotator cuff disease, specific subgroups of the disease, and the associations for the subgroups. Impingement was always accompanied by specific rotator cuff disease. Fifty percent of the patients with rotator cuff disease $(52 / 105)$ had multiple disorders.

Table 6.1 Frequencies of ultrasound-diagnosed rotator cuff disorders and predictive factors $(n=129)$

\begin{tabular}{|c|c|c|c|}
\hline Variable & $\mathrm{n} / \mathrm{n}(\%)$ & $\begin{array}{c}\text { Univariate analysis } \\
\text { OR }(95 \% \mathrm{Cl})\end{array}$ & p-value \\
\hline No disorder & $24 / 129(18.6)$ & & \\
\hline Rotator cuff disease present & $105 / 129(81.4)$ & & \\
\hline Age $\geq 40$ years & 96/106 (90.6) & $14.93(5.17-43.14)$ & $<.001$ \\
\hline Sex female & $56 / 129(43.4)$ & $0.89(0.36-2.16)$ & .79 \\
\hline Occupational risk & $40 / 129(31.0)$ & $0.88(0.34-2.26)$ & .79 \\
\hline \multicolumn{4}{|l|}{ Specific disorder } \\
\hline Calcific tendonitis & $65 / 129(50.4)$ & & \\
\hline with other rotator cuff disorder & $39 / 65 \quad(60.0)$ & & \\
\hline$\geq 40$ years & $63 / 106(59.4)$ & $15.38(3.43-69.04)$ & $<.001^{b}$ \\
\hline Tendinopathy & $37 / 129(28.7)$ & & \\
\hline with other rotator cuff disorder & $28 / 37 \quad(75.7)$ & & \\
\hline$\geq 40$ years & $33 / 106(31.1)$ & $2.15(0.68-6.81)$ & $.19^{\mathrm{b}}$ \\
\hline Bursitis & $26 / 129(20.2)$ & & \\
\hline with other rotator cuff disorder & $23 / 26 \quad(88.5)$ & & \\
\hline$\geq 40$ years & $26 / 106(24.5)$ & a & $.004^{\mathrm{b}}$ \\
\hline Partial-thickness tear & 25/129 (19.4) & & \\
\hline with other rotator cuff disorder & $16 / 25 \quad(64.0)$ & & \\
\hline$\geq 40$ years & $22 / 106(20.8)$ & $1.75(0.48-6.41)$ & $.56^{\mathrm{b}}$ \\
\hline ACJ-osteoarthritis & $16 / 129(12.4)$ & & \\
\hline with other rotator cuff disorder & $11 / 16 \quad(68.8)$ & & \\
\hline$\geq 40$ years & $16 / 106(15.1)$ & a & $.07^{\mathrm{b}}$ \\
\hline Full-thickness tear & $4 / 129(3.1)$ & & \\
\hline with other rotator cuff disorder & $4 / 4 \quad(100)$ & & \\
\hline$\geq 40$ years & $4 / 106(3.8)$ & a & $>.99^{\mathrm{b}}$ \\
\hline Impingement & $21 / 129(16.3)$ & & \\
\hline$\geq 40$ years & $20 / 106$ (18.9) & $5.12(0.65-40.23)$ & $0.12^{\mathrm{b}}$ \\
\hline
\end{tabular}

Note: One patient had a biceps tendosynovitis. ACJ, acromioclavicular joint; OR, odds ratio; ${ }^{\mathrm{a}}$ OR not provided, expected count in 1 of the cells is $0 ;{ }^{b}$ Fisher's exact test 
Table 6.2 displays the frequencies and combinations of multiple disorders observed. Linear regression analysis showed that the number of disorders significantly increased with increasing age $\left(R^{2}\right.$ 0.29). The univariate analysis showed that age was significantly associated with the presence of rotator cuff disease, which was confirmed by the results of the multivariate analysis (OR $1.13,95 \% \mathrm{Cl} 1.07-1.19)$.

Table 6.2 Frequencies of multiple ultrasound-diagnosed disorders in patients with rotator cuff disease $(n=105)$

\begin{tabular}{|c|c|c|c|}
\hline \multicolumn{2}{|c|}{ Number and combination of disorders } & \multirow{2}{*}{$\begin{array}{c}\text { No. } \\
36\end{array}$} & \multirow{2}{*}{$\begin{array}{c}(\%) \\
(34.3)\end{array}$} \\
\hline 2 & Disorders & & \\
\hline & Calcific tendonitis and tendinopathy & 13 & $(12.4)$ \\
\hline & Calcific tendonitis and bursitis & 8 & (7.6) \\
\hline & Tendinopathy and bursitis & 3 & (2.9) \\
\hline & Tendinopathy and ACJ-osteoarthritis & 2 & (1.9) \\
\hline & Partial-thickness tear and calcific tendonitis & 2 & (1.9) \\
\hline & Partial-thickness tear and bursitis & 2 & (1.9) \\
\hline & Calcific tendonitis and $\mathrm{ACJ}$-osteoarthritis & 2 & (1.9) \\
\hline & Full-thickness tear and calcific tendonitis & 1 & (0.9) \\
\hline & Full-thickness tear and bursitis & 1 & (0.9) \\
\hline & Full-thickness tear and ACJ-osteoarthritis & 1 & (0.9) \\
\hline & Bursitis and $\mathrm{ACJ}$-osteoarthritis & 1 & (0.9) \\
\hline \multirow[t]{7}{*}{3} & Disorders & 14 & $(13.3)$ \\
\hline & Partial-thickness tear, tendinopathy \& calcific tendonitis & 6 & (5.7) \\
\hline & Partial-thickness tear, bursitis \& calcific tendonitis & 2 & (1.9) \\
\hline & Partial-thickness tear, bursitis \& tendinopathy & 2 & (1.9) \\
\hline & Calcific tendonitis, bursitis \& ACJ-osteoarthritis & 2 & (1.9) \\
\hline & Partial-thickness tear, bursitis \& ACJ-osteoarthritis & 1 & (0.9) \\
\hline & Full-thickness tear, calcific tendonitis \& ACJ-osteoarthritis & 1 & (0.9) \\
\hline \multirow[t]{3}{*}{4} & Disorders & 2 & (1.9) \\
\hline & Calcific tendonitis, tendinopathy, bursitis \& partial-thickness tear & 1 & (0.9) \\
\hline & Calcific tendonitis, tendinopathy, bursitis \& ACJ-osteoarthritis & 1 & (0.9) \\
\hline
\end{tabular}

ACJ, acromioclavicular joint

\section{Discussion}

Our results suggest that age of 40 years and older is a strong predictor rotator cuff disease in patients complaining of acute shoulder pain in family medicine. More than $90 \%$ of patients with acute shoulder pain had one or more specific causes of rotator cuff disease detected on ultrasound imaging, with calcific tendonitis by far the most frequently observed. 
Our study was the first to prospectively assess the findings of ultrasound imaging in patients visiting their family physician with acute shoulder pain. In comparison with studies in secondary care, our study showed a higher prevalence of calcific tendonitis, and only a relatively small proportion of tears. ${ }^{5}$ These findings can be explained by the overrepresentation of patients in the diagnostic workup for surgery in secondary care populations.

Limitations of this study were that it lacked comparisons with the contralateral shoulder, reliability studies, a control group, and the relatively high number of participating radiologists $(n=13)$. The contralateral shoulder was scanned when findings were ambiguous, and $80 \%$ of all ultrasound procedures were performed by the six most experienced of the musculoskeletal radiologists, reflecting common care practice in The Netherlands, which thus increases the generalizability of our results.

For daily practice, it is important that ultrasound findings should always be weighed against the clinical context, as asymptomatic findings may be present. ${ }^{7}$ Our results show that ultrasound imaging enables family physicians to rationalize treatment, especially for patients aged 40 years and older. Importantly, it also provides an explanation for the complaints and informs about prognosis. For instance, calcific tendonitis is characterized by recurrent painful episodes. ${ }^{8}$ Given the low prevalence of full-thickness tears, unnecessary referrals to secondary care can be prevented. Patients with an inflammatory disorder, like bursitis or calcifications in the resorption phase, may benefit from corticosteroid injections. In the case of multiple causes of rotator cuff disease, patients can be treated according to the disorder most likely to be symptomatic, or additional therapeutic approaches can be initiated.

The clinical value of ultrasound imaging will depend on whether patient outcomes change. Further studies are needed to prove that early ultrasound imaging in the diagnostic work-up improves patient outcome. ${ }^{6}$ 


\section{References}

1. van der Windt DA, Koes BW, Boeke AJ, Devillé W, De Jong BA, Bouter LM. Shoulder disorders in general practice: prognostic indicators of outcome. Br J Gen Pract. 1996;46:519-523.

2. Hanchard NC, Lenza M, Handoll HH, Takwoingi Y. Physical tests for shoulder impingements and local lesions of bursa, tendon or labrum that may accompany impingement. Cochrane Database Syst Rev. 2013;4:CD007427.

3. NHS/NICE CKSb. Shoulder pain guideline. Available at: http://www.cks.nhs.uk/shoulder_pain.

4. Winters JC, van der Windt DAWM, Spinnewijn WEM, et al. Shoulder pain guideline of the Dutch College of General Practitioners (in Dutch). Huisarts Wet. 2008;51:555-565.

5. Ottenheijm RPG, Jansen MJ, Staal JB, et al. Accuracy of diagnostic ultrasound in patients with suspected subacromial disorders: a systematic review and meta-analysis. Arch Phys Med Rehabil. 2010;91: 1616-1625.

6. Ottenheijm RP, Joore MA, Walenkamp GH, et al. The Maastricht Ultrasound Shoulder pain Trial (MUST): ultrasound imaging as a diagnostic triage tool to improve management of patients with non-chronic shoulder pain in primary care. BMC Musculoskelet Disord. 2011;12:154.

7. Schibany $\mathrm{N}$, Zehetgruber $\mathrm{H}$, Kainberger $\mathrm{F}$, et al. Rotator cuff tears in asymptomatic individuals: a clinical and ultrasonographic screening study. Eur J Radiol. 2004;51:263-268.

8. Carcia CR, Scibek JS. Causation and management of calcific tendonitis and periarthritis. Curr Opin Rheumatol. 2013;25:204-209. 


\section{Chapter}

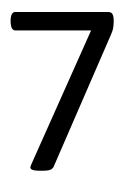

Clinical effectiveness of ultrasound tailored treatment in patients with acute shoulder pain in general practice: a pragmatic randomised controlled trial (Maastricht Ultrasound Shoulder pain Trial)

Ramon PG Ottenheijm, Jochen WL Cals, Bjorn Winkens, René E Weijers, Rob A de Bie, Geert-Jan Dinant 


\section{Abstract}

\section{Objectives}

To determine the clinical effectiveness of ultrasound tailored treatment in patients with acute subacromial disorders.

\section{Design}

Pragmatic, randomised controlled trial.

\section{Setting}

Dutch general practice.

\section{Participants}

Patients aged 18 to 65 years with unilateral shoulder pain lasting fewer than three months and no previous treatment, in whom the general practitioner, based on medical history taking and physical examination, suspected a subacromial disorder.

\section{Interventions}

All patients underwent ultrasound imaging of the affected shoulder. Patients who were still symptomatic after a qualification period of 2 weeks with standard treatment were randomised to treatment tailored to ultrasound diagnosis (disclosure of the ultrasound diagnosis) or usual care (non-disclosure of the ultrasound diagnosis). Patients were stratified for age at a cut-off of $\geq 50$ years.

\section{Main outcome measures}

Primary outcome was patient perceived recovery at 1-year measured with the Global Perceived Effect questionnaire. Secondary outcomes included the Shoulder Pain Score, the Shoulder Disability Questionnaire, The Euroqol five-item Quality of life questionnaire, and one-year health care use. Follow-up was performed using postal questionnaires at 3, 6, 9 and 12 months. Analysis was done by intention-to-treat using likelihood based method for missing outcome data.

\section{Results}

111 patients were randomised, of whom 56 were allocated to ultrasound tailored treatment and 55 to usual care. Baseline characteristics were comparable. At 1 year, there was no significant difference between the groups in recovery: $72.5 \%(37 / 51)$ in the ultrasound tailored treatment group vs. $60 \%(30 / 50)$ in the usual care group, Odds Ratio $1.86(95 \% \mathrm{Cl} 0.79$ to $4.36 ; \mathrm{p}=0.15)$. Logistic mixed models analysis did not alter the results, Odds Ratio $2.24(95 \% \mathrm{Cl} 0.72$ to $6.89, \mathrm{p}=0.16)$. Secondary outcome measures also showed no significant differences at 1 year.

\section{Conclusions}

Ultrasound tailored treatment in patients with acute shoulder pain without previous treatment in whom the GP suspected a subacromial disorder does not significantly improve outcome at 1 year. Furthermore, this treatment did not influence healthcare use on study group level. From a clinical point of view, GPs should be informed that ultrasound imaging in patients with acute shoulder pain does not provide additional information to improve patient outcome. 


\section{Introduction}

General practitioners (GPs) are frequently consulted by patients with shoulder pain. ${ }^{1}$ After initial presentation to the GP, re-consultation takes place in $50 \%$ of the cases. ${ }^{2}$ About half of all patients who visit their GP with a new episode of shoulder pain report persistent complaints after six months, and up to $40 \%$ still have pain and disability after 1 year, $^{3-5}$ while recurrence rates vary from $31 \%$ after 1 year to $85 \%$ after 12 years. ${ }^{6-8}$ These finding suggest that shoulder pain frequently progresses to a chronic disorder. Shoulder pain is a symptom, not a diagnosis. Many studies have aimed at finding the best initial treatment for this symptom without success. Subacromial disorders including rotator cuff disorders and subacromial-subdeltoid bursitis are the most common cause of shoulder pain seen by GPs. ${ }^{9}$ In general practice, accurate diagnosis of shoulder pain is difficult because the findings from medical history and physical examination have often shown poor correlation with the underlying disorder. ${ }^{10-12}$ Therefore, British and Dutch guidelines for shoulder pain advise GPs to start treatment based on patients' signs and symptoms rather than on a the actual disorder, while diagnostic imaging, e.g. ultrasound, is only advised in persistent cases. ${ }^{9,13}$ GPs experience the current diagnostic process as complex, and the use of diagnostic ultrasound as helpful in establishing a more accurate diagnosis. ${ }^{14-19}$ Accuracy studies showed that diagnostic ultrasound is accurate for evaluating subacromial disorders. ${ }^{20-22}$ Moreover, the full spectrum of subacromial disorders is observed in patients with shoulder pain presenting in general practice. ${ }^{23-25}$ For each of these disorders, evidence based treatments are available, such as physiotherapy in case of tendinopathy or corticosteroid injections in case of bursitis. ${ }^{26,27}$ This implies that stratification of patients into diagnostic subgroups potentially allows for more tailored treatment than currently applied. For instance, in general practice patients with low back pain, a stratified management approach in which prognostic screening and treatment targeting were combined improved patient outcome. ${ }^{28}$

In daily general practice, combining clinical information with ultrasound diagnosis is potentially helpful to tailor treatment in patients with shoulder pain. So far, no pragmatic trial has evaluated this test-treatment approach for shoulder pain in general practice. We hypothesise that the currently advocated stepwise approach in guidelines can be improved by applying ultrasound tailored treatment as early management strategy in acute shoulder pain. The objective of this study was to determine the clinical effectiveness of ultrasound tailored treatment in patients with acute subacromial disorders. 


\section{Methods}

\section{Design overview}

The design of this pragmatic randomised controlled trial (RCT), the Maastricht Ultrasound Shoulder pain Trial, was published in detail elsewhere with open access. ${ }^{29}$ After a qualification period of two weeks, patients who were still symptomatic with standard treatment entered the RCT with a 50 week follow-up period comparing two treatment strategies for patients with shoulder pain in whom the GP suspected a subacromial disorder: treatment tailored to ultrasound diagnosis and usual care (Figure 7.1). The 2-week qualification period was used to perform the diagnostic ultrasound and aimed to filter out patients with a favourable natural course. During this period patients received treatment according to the guidelines for shoulder pain of the Dutch College of General Practitioners: paracetamol or NSAIDs and advice regarding activities of daily living, work, hobbies, and sports. ${ }^{9}$ The primary end point was 1-year patientperceived recovery. Our reporting follows the Consolidated Standards of Reporting Trials (CONSORT) extension for pragmatic trials. ${ }^{30}$ The study was approved by the Medical Ethics Committee of the Maastricht University Medical Centre, and the trial was registered at the Netherlands Trial Register (NTR2403).

\section{Patients and setting}

To be included patients had to be aged between 18 and 65 years, have unilateral shoulder pain upon abduction with a painful arc, symptoms having lasted less than three months and no other episodes of shoulder pain in the previous 12 months. Exclusion criteria were 1) consultation or treatment for shoulder pain in the past three months; 2) glenohumeral external rotation range of motion less than 45 degrees as this is a reason to suspect a glenohumeral disorder like osteoarthritis or a frozen shoulder; 3 ) history of fractures of the proximal humerus or acromion; 4) dislocation and/or surgery of the affected shoulder; 5 ) shoulder complaints caused by rheumatic disease; 6) suspected referred complaints or extrinsic cause; 7) history of depressive or anxiety disorders, or pain catastrophizing; 8) inability to complete a questionnaire independently; 9) inability to provide informed consent; 10) or involved in disability or liability procedures. All patients gave written informed consent. Initially, 21 GPs working in 11 practices in the Westelijke Mijnstreek, a region in the southern part of the Netherlands, recruited eligible patients. These GPs were asked to include sequential eligible patients within regular consultation hours. After two years, all 80 GPs in the aforementioned region were asked to recruit patients. All GPs received oral and written instructions. 


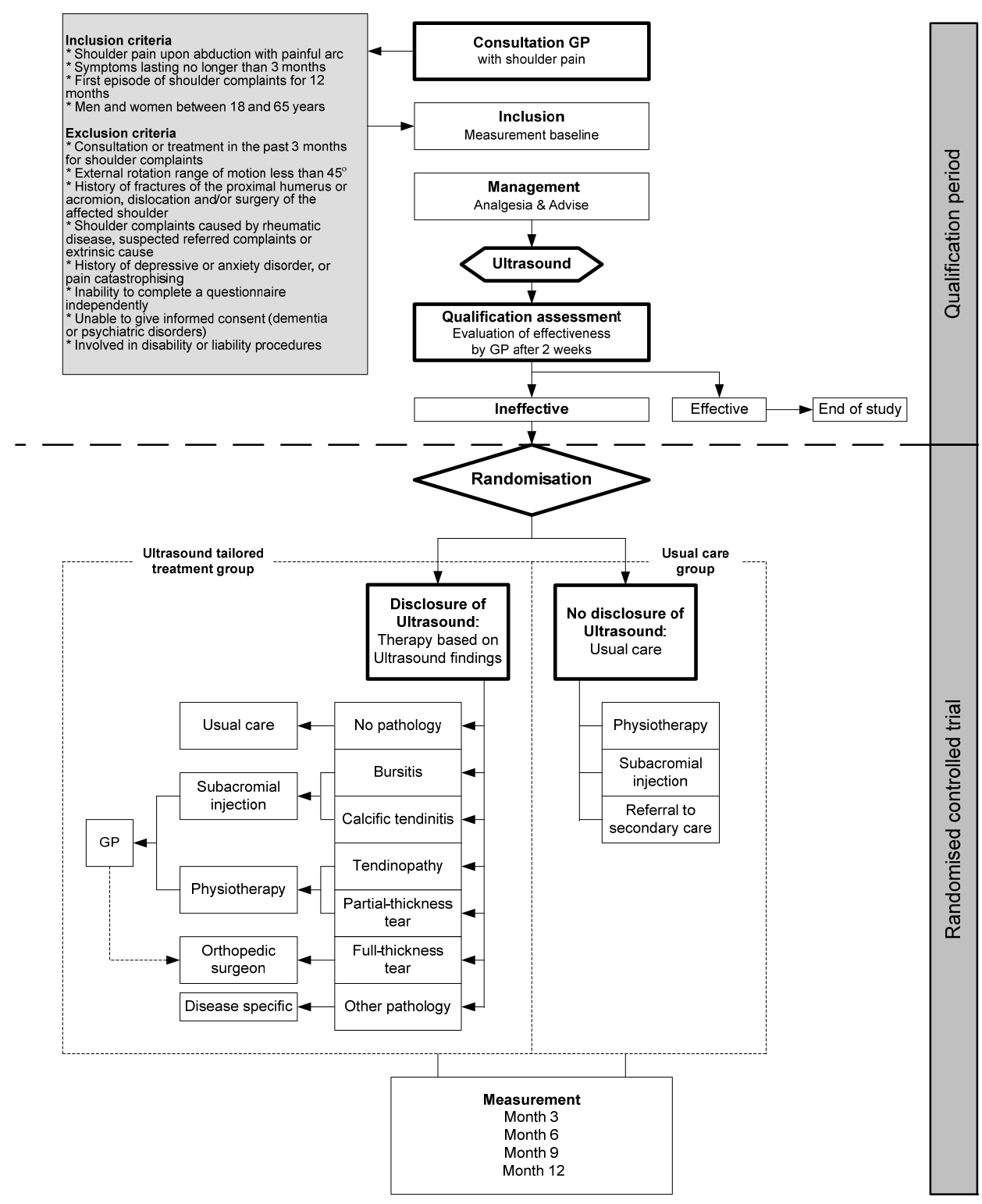

Figure 7.1 Flowchart of the study 


\section{Interventions}

During the 2-week qualification period all patients were referred for diagnostic ultrasound of the shoulder to the radiology department of the Orbis Medical Centre in Sittard-Geleen. Experienced musculoskeletal radiologists performed all procedures using a standardized protocol, which included the ultrasound examination technique and criteria for pathology. This protocol has been described in detail elsewhere. ${ }^{29}$ The two study arms were treatment tailored to the ultrasound diagnosis or usual care. The GP provided the allocated treatments. Because we hypothesised that physiotherapy could be a frequently employed treatment, the physiotherapists in the study area were trained in applying the evidence based approach for subacromial disorders released by the Royal Dutch Society for Physical Therapy. ${ }^{27}$

\section{Ultrasound tailored treatment}

Key feature of this intervention was disclosure of the ultrasound diagnosis to the GP in order to tailor treatment. GPs treated patients according to the advised evidence based, tailored treatment steps as presented in Figure 7.1 and in detail published in the study protocol. ${ }^{29}$ Advised treatment modalities depending on the ultrasound diagnosis were subacromial corticosteroid injections in case of bursitis or calcific tendonitis, referral to a physiotherapist in case of tendinopathy or partial-thickness tendon tear, and an orthopedic surgeon in case of full-thickness tendon tears.

In case there were no abnormal ultrasound findings, usual care according to the guidelines for shoulder pain was provided. In cases where multiple ultrasound diagnoses were present, the most relevant diagnosis was selected on the basis of the clinical findings. Motivated and within the recommendations made in the guidelines for shoulder pain, GPs were allowed to deviate from the advised treatment steps.

\section{Usual care}

In the control group the ultrasound diagnosis was not disclosed, and therefore, usual care according to the guidelines for shoulder pain was applied. ${ }^{9}$ It consisted of a pragmatic, stepwise approach; a wait-and-see policy with advice and analgesia for another 2 weeks; corticosteroid injections and referral to a physiotherapist were advised as options in persisting cases, depending on the level of pain and functional limitations respectively; referral to a hospital specialist was advised if conservative treatment failed. $^{9}$ 


\section{Randomisation and blinding}

Based on the qualification assessment at two weeks, unrecovered patients qualified for the RCT. Recovery was measured by the Global Perceived Effect questionnaire, a valid measure for patients with musculoskeletal disorders. ${ }^{31}$ It consists of a one-item score concerning recovery following treatment, measured on a seven-point ordinal scale. Patients were considered recovered when they reported to be much improved or fully recovered. ${ }^{31}$ Using an online application, developed at the centre for data and information management of Maastricht University, patients were randomly assigned by central block randomisation (blocks of 4 ) to one of the study arms after stratification for age (cut-off $\geq 50$ years).

Neither the patient nor the GP could be blinded for allocated treatment. However, ultrasound diagnosis were only disclosed to GPs of those patients in the ultrasound tailored treatment group. In that case, the GP received the ultrasound diagnosis and the advised tailored treatment strategies. In case of usual care, the GP only received the treatment as advised in the guidelines for shoulder pain, while the ultrasound diagnosis was disclosed to the GP at the end of patients' follow-up period. Radiologists were not allowed to communicate with the patient about the ultrasound imaging results.

\section{Outcomes}

Follow-up was done by postal questionnaires at baseline, 3, 6, 9 and 12 months. The primary outcome measure was the patient-perceived recovery using the Global Perceived Effect questionnaire. ${ }^{31}$ Secondary outcomes included the experienced shoulder pain using the Shoulder Pain Score, the performance of daily activities using the Shoulder Disability Questionnaire, and the health-related quality of life using the Euroqol five-item quality of life questionnaire (EQ-5D). The Shoulder Pain Score questionnaire consists of six pain symptom questions and a 10 -point scale. ${ }^{32}$ The Shoulder Pain Score has been proved to be a useful instrument to follow the course of the disorder over time, and gives an indication when a patient feels cured. The score can range from 7 to 28 with a higher score indicating more pain. The Shoulder Disability Questionnaire contains 16 questions and is a useful discriminative instrument, especially in the primary care setting. ${ }^{33}$ The score ranges from 0 to 100 with a higher score indicating a more severe disability. The EQ-5D is one of the most used generic measures to quantify health related quality of life in patients with musculoskeletal disorders, ${ }^{34,35}$ and consists of two sections. The first section comprises five questions regarding five dimensions of health. Calculation of the index score is performed according to the British recommendations and ranges from -1 to 1 , higher scores 
indicating better quality of life. ${ }^{36}$ The second section is a visual analogue scale ranging from 0 (worst imaginable health state) to 100 (best imaginable health state). At inclusion, patients of whom the GP doubted about pain catastrophizing behavior were given the Pain Catastrophizing Scale. This is a 13-item self-report scale to measure pain catastrophizing and the score can range from 0 to 52 with a higher score indicating a higher level of catastrophizing. We made use of a cut-off value of 20 points. ${ }^{37}$ Patients were excluded by the research team if their score was $>20$ points.

To measure the 1-year healthcare use, we obtained the electronic patient records of all patients by contacting the GP and used the questionnaires. In case patients were referred to a physiotherapist, the number of visits were collected.

\section{Sample size}

Based on the available literature we assumed that after one year the recovery rate of patients with shoulder pain would be $60 \%{ }^{3}$ We estimated a $20 \%$ improvement in recovery rate as the minimal clinical important difference, and therefore calculated our sample size on the ability to detect a difference in study arms of $20 \%$ (60\% versus $80 \%$ ) or more on recovery rate. Using these data, we estimated that we needed 81 patients per study arm to show a significant difference at the $5 \%$ level with $80 \%$ power. With allowance for a $10 \%$ drop-out rate, we therefore required 90 patients per study arm. $^{38,39}$ With the expectation that the qualification period would filter out $20 \%$ of the patients we needed to include 226 patients in total.

\section{Statistical analysis}

Numerical variables were expressed as means and standard deviations, and categorical variables as frequencies and percentage. Differences between groups were analysed using independent-samples t-test or Mann-Whitney U-test where appropriate. The Chisquare test was used for categorical variables.

For each analysis, either group (logistic regression) or group, time and group*time (mixed models) were used as fixed factors (categorical). The random part of the mixed models was chosen using Akaike's Information Criterion. For the primary outcome measure Global Perceived Effect at 12 months, analysis was done using a logistic regression analysis with correction for the stratification variable age (cut-off at 50 years). A three level logistic mixed model to correct and account for age (cut-off at 50 years) and variation at the level of the GP practice, patient, and repeated observations with data from four time points $(3,6,9$ and 12 months) was used. The secondary outcomes Shoulder Pain Score, Shoulder Disability Questionnaire and EQ-5D were analysed using a linear mixed model correcting and accounting for age and 
variation at the level of GP practice, patient and repeated observations with data from four time points $(0,3,6$ and 12 months). Shoulder-related health care resource during one year follow-up was analysed using a logistic regression analysis with correction for the stratification variable age (cut-off at 50 years).

The primary analysis of the data was undertaken according to the intention-to-treat principle, and included all patients. In addition, a per protocol analysis of complete cases for the primary outcome at 12 months was performed to estimate the impact on the effect of loss to follow-up and protocol deviations. Deviations of the protocol were defined as not being treated according to the advised treatment steps in the ultrasound tailored treatment group or in case of any form of information bias in the usual care group; the radiologist discussed the ultrasound diagnosis with the patient or the GP unintentionally received the ultrasound diagnosis. To handle single item missing data, a sensitivity analysis based on best and worst case scenarios (highest and lowest scores) was performed. No multiple imputation was used if whole questionnaires were missing, since missing outcome data were dealt with using a likelihood based approach, assuming missing at random.

Putative prognostic factors at baseline, sex, onset of shoulder pain, dominant shoulder affected and concomitant neck complaints, as well as recruitment strategy were added as covariate to the mixed model. Due to the expected small number of non-recovered patients, this correction was only applied to the numerical outcomes.

All analyses were performed using IBM SPSS Statistics for Windows, version 21.0 (IBM Corp., Armonk, NY, USA). A p-value $\leq 0.05$ was considered statistically significant.

\section{Results}

Over a 38-month recruitment period (November 2010 to December 2013), 129 patients were included in $26 \mathrm{GP}$ practices. The flow of patients through the study is presented in Figure 7.2. Eighteen patients $(14 \%, 18 / 129)$ recovered during the 2 -week qualification period, resulting in 111 randomised patients; 56 were allocated to ultrasound tailored treatment and 55 to usual care. Ten patients were lost to follow-up at 1 year; three patients only returned the baseline questionnaire, while seven patients did not return any questionnaire after 3 months. Of these patients, one (control group) could be contacted and reported to be recovered at 6 months but refused to return future questionnaires, while the remaining nine patients could not be reached.

Baseline characteristics were similar in both randomised groups (Table 7.1). Two patients were prompted by their GP to complete the Pain Catastrophizing Scale, and both were classified as not pain catastrophizing. 


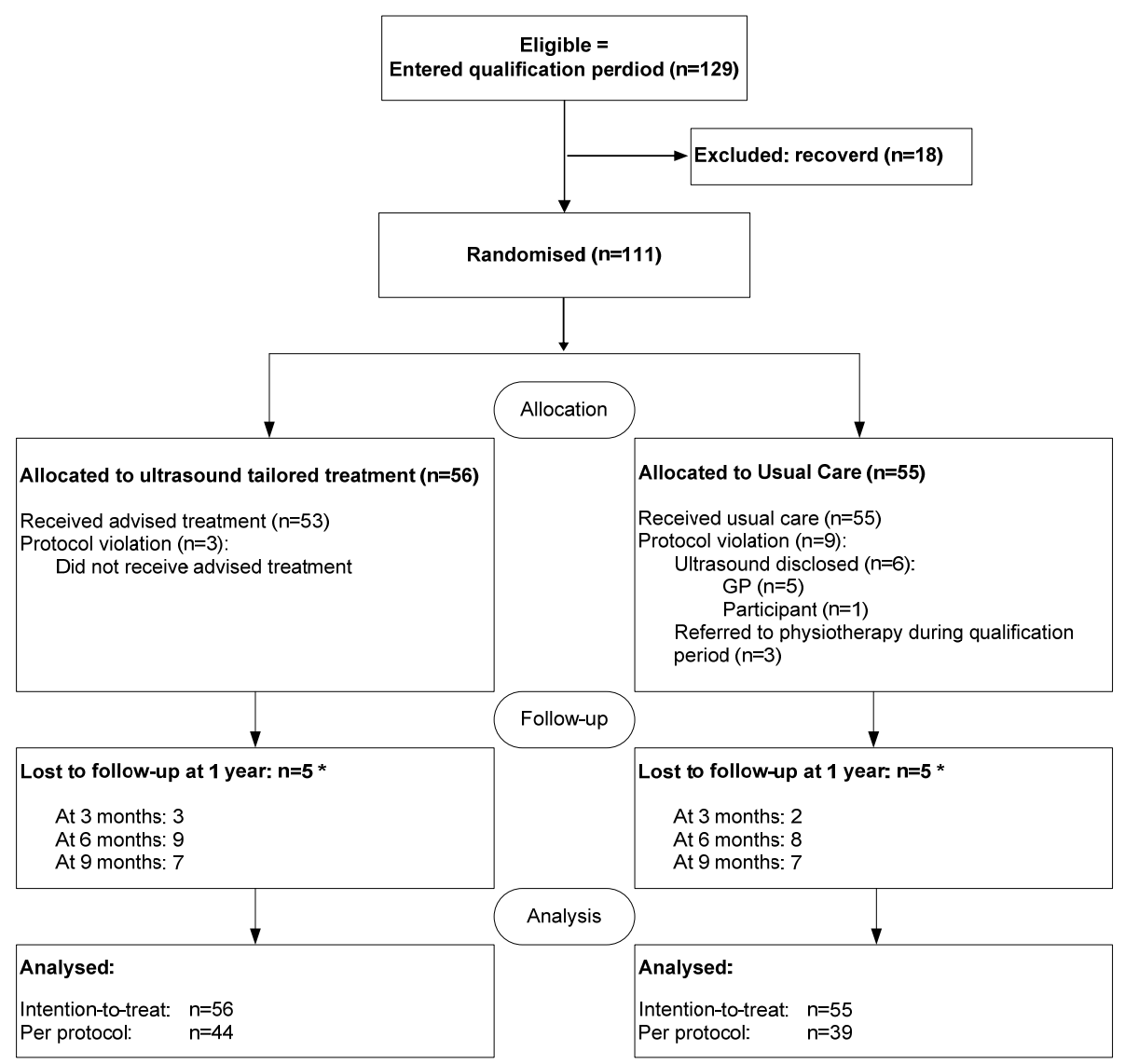

* Lost to follow-up was reported for each time point. Some participants missed 1 time point, but were followed up at a different time point

Figure 7.2 Flow of patients through study

\section{Patient perceived recovery}

Although more patients in the ultrasound tailored treated group perceived to be recovered after 1 year according to the patients' Global Perceived Effect assessment $(72.5 \%(37 / 51)$ vs. $60 \%(30 / 50)$, Odds Ratio $1.86,(95 \% \mathrm{Cl} 0.79$ to 4.36$))$, this difference was not significant $(p=0.15)$. The intention-to-treat analysis of this primary outcome measure is presented in Table 7.2, as well as the recovery rates after 3, 6, and 9 months. Logistic mixed model analysis adjusting for age (cut-off at 50 years) and variation at the level of the GP practice, patient, and repeated observations with data 
from four time points $(3,6,9$ and 12 months) showed similar results (Odds Ratio 2.24, $95 \% \mathrm{Cl} 0.72$ to $6.89, \mathrm{p}=0.16$ ) for patient perceived recovery at 1 year.

Table 7.1 Baseline characteristics

\begin{tabular}{lccc}
\hline Variables & $\begin{array}{c}\text { Ultrasound Tailored } \\
\text { Treatment } \\
(\mathrm{n}=56)\end{array}$ & $\begin{array}{c}\text { Usual Care } \\
(\mathrm{n}=55)\end{array}$ & $\begin{array}{c}\text { Recovered before } \\
\text { randomisation } \\
(\mathrm{n}=18)\end{array}$ \\
\hline Demographic variables & $49.0(9.9)$ & $49.4(10.9)$ & $48.6(12.4)$ \\
Age, mean (SD) & $26(46.4)$ & $20(36.4)$ & $10(55.6)$ \\
Female, $\mathrm{n}(\%)$ & & & $2.1(1.8)$ \\
Specific disease variables & $6.2(3.8)$ & $5.5(3.5)$ & $13(72.2)$ \\
Duration of pain in weeks (SD) & $24(42.9)$ & $28(50.9)$ & $6(33.3)$ \\
Acute onset, $\mathrm{n}$ (\%) & $20(35.7)$ & $14(25.5)$ & $9(50.0)$ \\
Concomitant neck complaints, $\mathrm{n}(\%)$ & $33(58.9)$ & $37(67.3)$ & $11(61.1)$ \\
Dominant shoulder affected, $\mathrm{n}(\%)$ & $26(46.4)$ & $28(50.9)$ & $5(27.8)$ \\
Ultrasound findings, $\mathrm{n}$ (\%) & $16(28.6)$ & $16(29.1)$ & $2(11.1)$ \\
Calcific tendonitis & $14(25.0)$ & $10(18.2)$ & $1(5.6)$ \\
Tendinopathy & $11(19.6)$ & $13(23.6)$ & $1(5.6)$ \\
Bursitis & $0(0.0)$ & $3(5.5)$ & $2(11.1)$ \\
Partial-thickness tears & $12(21.4)$ & $7(12.7)$ & \\
Full-thickness tears & & & $4(22.2)$ \\
Impingement & $12(21.4)$ & $8(14.5)$ & $7(38.9)$ \\
Number of disorders, $\mathrm{n}(\%)$ & $20(35.7)$ & $26(47.3)$ & $7(38.9)$ \\
$\quad$ No disorder & $24(42.9)$ & $21(38.2)$ & \\
1 disorder & & & \\
\hline 2 disorders & & & \\
\hline
\end{tabular}

SD, Standard Deviation

\section{Secondary outcomes}

After 1 year the mean differences in the Shoulder Pain Score $(1.7$ points, $95 \% \mathrm{Cl}-3.9$ to 0.5 ) and Shoulder Disability Questionnaire (6.9 points, $95 \% \mathrm{Cl}-19.9$ to 6.1 ) were in favour of the ultrasound tailored treated group, but these differences were not significant ( $p=0.15$ and $p=0.29$, respectively). Also health-related quality of life, measured with the EQ-5D and EQ-5D VAS, was not significantly different between groups (Table 7.3). Analysis adjusting for the putative prognostic factors did not alter the results. We did not find any significant differences in the proportion of patients referred to physiotherapy (59\% of patients receiving ultrasound tailored treatment vs. $64 \%$ of patients receiving usual care), their mean number of physiotherapy sessions (12.9 vs. 12.4 for ultrasound tailored treatment and usual care, respectively), corticosteroid injections or referrals to secondary care (39\% vs. $31 \%$ and $20 \%$ vs. $13 \%$ for ultrasound tailored treatment and usual care, respectively). Shoulder-related health care use at 1 year between the two study arms is shown in Table 7.4. No adverse events or side effects were reported. 
Table 7.2 Primary outcome measure, Global Perceived Effect

\begin{tabular}{|c|c|c|c|c|c|c|c|c|c|c|c|}
\hline Recovered & & $\begin{array}{l}\text { ound } \\
\text { ored } \\
\text { ment }\end{array}$ & Usu & al care & Between & aro & difference & & & & \\
\hline & $\%$ & & 0 & & Diff. (\%) & $\mathrm{OR}^{\mathrm{a}}$ & $95 \% \mathrm{Cl}$ & $p$-value & $O R^{b}$ & $95 \% \mathrm{Cl}$ & $p$-value \\
\hline 3 months & 41.5 & $(22 / 53)$ & 32.1 & $(17 / 53)$ & 9.4 & 1.52 & $0.67-3.38$ & .30 & 2.18 & $0.75-6.37$ & .15 \\
\hline 6 months & 46.8 & $(22 / 47)$ & 44.7 & $(21 / 47)$ & 2.1 & 1.10 & $0.47-2.48$ & .82 & 1.15 & $0.39-3.50$ & .80 \\
\hline 9 months & 53.1 & $(26 / 49)$ & 60.4 & $(29 / 48)$ & -7.3 & 0.75 & $0.33-1.73$ & .50 & 0.59 & $0.20-1.80$ & .35 \\
\hline 12 months & 72.5 & $(37 / 51)$ & 60.0 & $(30 / 50)$ & 12.5 & 1.86 & $0.79-4.36$ & .15 & 2.24 & $0.72-6.89$ & .16 \\
\hline
\end{tabular}

Diff, Difference; OR, Odds Ratio; ${ }^{a}$ Based on logistic regression analysis correcting for age (stratification variable, cut-off 50 years); ${ }^{b}$ Based on logistic mixed models analysis correcting for age (stratification variable, cut-off 50 years) and variation at the level of the GP practice, patient, and repeated observations. The level- 3 variance (GP practice) was equal to $0 ; 95 \% \mathrm{Cl}, 95 \%$ Confidence Interval

Table 7.3 Secondary outcome measures

\begin{tabular}{|c|c|c|c|c|c|c|c|c|c|c|}
\hline \multirow[t]{3}{*}{$\begin{array}{l}\text { Outcome } \\
\text { variable }\end{array}$} & \multicolumn{3}{|c|}{$\begin{array}{c}\text { Ultrasound Tailored } \\
\text { Treatment }\end{array}$} & \multicolumn{3}{|c|}{ Usual care } & \multicolumn{4}{|c|}{ Between-group difference } \\
\hline & \multirow[b]{2}{*}{$\mathrm{N}$} & \multirow[b]{2}{*}{ Mean } & \multirow[b]{2}{*}{ (SD) } & \multirow[b]{2}{*}{$\mathrm{N}$} & \multirow[b]{2}{*}{ Mean } & \multirow[b]{2}{*}{$(S D)$} & \multirow{2}{*}{$\begin{array}{c}\text { Mean } \\
\text { difference }^{\text {a }}\end{array}$} & \multicolumn{2}{|c|}{$95 \% \mathrm{Cl}$} & \multirow[b]{2}{*}{$\mathrm{p}$-value } \\
\hline & & & & & & & & lower & upper & \\
\hline \multicolumn{11}{|l|}{ SPS } \\
\hline Baseline & 56 & 20.6 & $(4.1)$ & 55 & 19.5 & $(4.3)$ & & & & \\
\hline 3 months & 53 & 14.3 & $(5.1)$ & 53 & 15.2 & $(5.5)$ & -2.1 & -4.2 & 0.1 & .06 \\
\hline 6 months & 47 & 13.1 & $(4.7)$ & 47 & 13.5 & $(5.4)$ & -1.7 & -3.8 & 0.5 & .13 \\
\hline 12 months & 51 & 11.1 & $(5.3)$ & 50 & 11.5 & $(4.8)$ & -1.7 & -3.9 & 0.5 & .12 \\
\hline \multicolumn{11}{|l|}{ SDQ } \\
\hline Baseline & 56 & 69.6 & $(17.3)$ & 55 & 68.3 & $(17.2)$ & & & & \\
\hline 3 months & 53 & 47.2 & $(32.4)$ & 53 & 49.4 & $(29.8)$ & -4.3 & -14.9 & 6.3 & .42 \\
\hline 6 months & 47 & 40.7 & (29.7) & 47 & 36.3 & (27.1) & 3.2 & -8.1 & 14.4 & .58 \\
\hline 12 months & 51 & 24.3 & $(30.2)$ & 50 & 31.0 & (29.7) & -6.9 & -19.9 & 6.1 & .29 \\
\hline \multicolumn{11}{|l|}{ EQ-5D } \\
\hline Baseline & 56 & 0.68 & $(0.20)$ & 55 & 0.73 & $(0.22)$ & & & & \\
\hline 3 months & 53 & 0.76 & $(0.21)$ & 53 & 0.79 & $(0.22)$ & 0.01 & -0.07 & 0.09 & .74 \\
\hline 6 months & 47 & 0.83 & $(0.18)$ & 47 & 0.84 & $(0.18)$ & 0.05 & -0.04 & 0.13 & .26 \\
\hline 12 months & 51 & 0.81 & $(0.27)$ & 50 & 0.87 & $(0.16)$ & 0.002 & -0.10 & 0.10 & .97 \\
\hline \multicolumn{11}{|l|}{ EQ-5D VAS } \\
\hline Baseline & 56 & 69.4 & $(15.2)$ & 55 & 69.9 & $(14.2)$ & & & & \\
\hline 3 months & 53 & 72.6 & $(17.6)$ & 53 & 73.1 & $(13.7)$ & 0.13 & -5.16 & 4.90 & .96 \\
\hline 6 months & 47 & 78.4 & $(12.0)$ & 47 & 76.5 & $(13.7)$ & 2.82 & -2.64 & 8.29 & .31 \\
\hline 12 months & 51 & 78.3 & $(15.8)$ & 50 & 77.4 & $(14.5)$ & 1.62 & -4.76 & 8.00 & .62 \\
\hline
\end{tabular}

SD, Standard Deviation; 95\% Cl, 95\% Confidence Interval; SPS, Shoulder Pain Score 7-28 (28=most pain); SDQ, Shoulder Disability Questionnaire 0-100 (100=most severe disability); EQ-5D, Euroqol five-item quality of life questionnaire tariff -1 to 1 (1=highest health related quality of life); EQ-5D VAS, Visual Analogue Scale 0-100 (100=best health status); ${ }^{a}$ Linear mixed models analysis corrected for baseline and age (stratification variable, cut-off 50 years) and variation at the level of the GP practice, patient, and repeated observations. The level-3 variance (GP practice) was equal to 0 for SPS and EQ-5D 
Table 7.4 Use of healthcare resources during 1 year follow-up

\begin{tabular}{|c|c|c|c|c|c|c|c|}
\hline \multirow{2}{*}{$\begin{array}{l}\text { Healthcare resource } \\
\text { GP re-consultation, } \mathrm{n}(\%)\end{array}$} & \multicolumn{2}{|c|}{$\begin{array}{l}\text { Ultrasound Tailored } \\
\text { Treatment } \\
n=54^{b}\end{array}$} & \multicolumn{2}{|c|}{$\begin{array}{l}\text { Usual care } \\
\text { n=55 }\end{array}$} & \multirow{2}{*}{$\begin{array}{l}\mathrm{OR}^{\mathrm{a}} \\
1.05\end{array}$} & \multirow{2}{*}{$\begin{array}{c}95 \% \mathrm{Cl} \\
0.49-2.25\end{array}$} & \multirow{2}{*}{$\begin{array}{r}\mathrm{p} \text {-value } \\
.90\end{array}$} \\
\hline & 24 & (43) & 30 & (55) & & & \\
\hline No. of re-consultations (mean, SD) & 1.7 & $(0.9)$ & 2.1 & $(1.3)$ & & & .26 \\
\hline Diagnostic imaging ordered by GP, $\mathrm{n}(\%)^{\mathrm{c}}$ & 56 & $(100)$ & 13 & $(24)$ & 0.58 & $0.17-2.04$ & .40 \\
\hline Plain radiography, $\mathrm{n}(\%)$ & 1 & $(2)$ & 6 & (11) & 1.02 & $0.21-4.92$ & .98 \\
\hline Ultrasound imaging, $\mathrm{n}(\%)^{\mathrm{c}}$ & 56 & $(100)$ & 12 & (22) & 0.72 & $0.20-2.60$ & .62 \\
\hline Physiotherapist referral, $\mathrm{n}(\%)$ & 33 & (59) & 35 & (64) & 0.83 & $0.38-1.81$ & .64 \\
\hline No. of physiotherapy sessions (mean, SD) ${ }^{d}$ & 12.9 & (9.0) & 12.4 & $(10.2)$ & & & .64 \\
\hline Medication used, $\mathrm{n}(\%)$ & 31 & (55) & 36 & (65) & 1.59 & .47 & .25 \\
\hline GP prescription, $\mathrm{n}(\%)$ & 12 & (21) & 21 & (38) & 0.85 & 0.37-1.95 & .70 \\
\hline отC, n (\%) & 25 & (45) & 27 & (49) & 1.17 & $0.55-2.49$ & .69 \\
\hline No. of GP prescriptions (mean, SD) & 1.6 & $(1.2)$ & 1.4 & $(0.7)$ & & & 1.00 \\
\hline Corticosteroid injections by GP, $\mathrm{n}(\%)$ & 22 & (39) & 17 & (31) & 1.27 & $0.57-2.82$ & .56 \\
\hline No. of injections (mean, SD) & 1.4 & $(0.67)$ & 1.5 & $(0.51)$ & & & .50 \\
\hline Secondary care referral, $\mathrm{n}(\%)$ & 11 & (20) & 7 & (13) & 2.23 & $0.73-6.82$ & .16 \\
\hline Surgery, n (\%) & 1 & $(2)$ & 3 & (5) & 0.74 & $0.10-5.54$ & .77 \\
\hline
\end{tabular}

OR, Odds Ratio; 95\% Cl, Confidence Interval; SD, Standard Deviation; GP, General Practitioner; OTC, over-thecounter medication; ${ }^{a}$ Logistic regression analysis corrected for age (stratification variable, cut-off 50 years); ${ }^{\mathrm{b}}$ One patient gave no consent to obtain her patient record, and one GP did not deliver the patient record; ${ }^{c}$ Including intervention ultrasound; ${ }^{d}$ From five patients in each group the number is lacking

\section{Sensitivity analysis}

Per protocol analyses of the primary outcome measure as well as the sensitivity analyses with best and worst case scenarios to handle single item missing data of the secondary outcome measures, produced similar effects as in the intention to treat analysis (supplementary Table 7.5 to inform reviewers). 


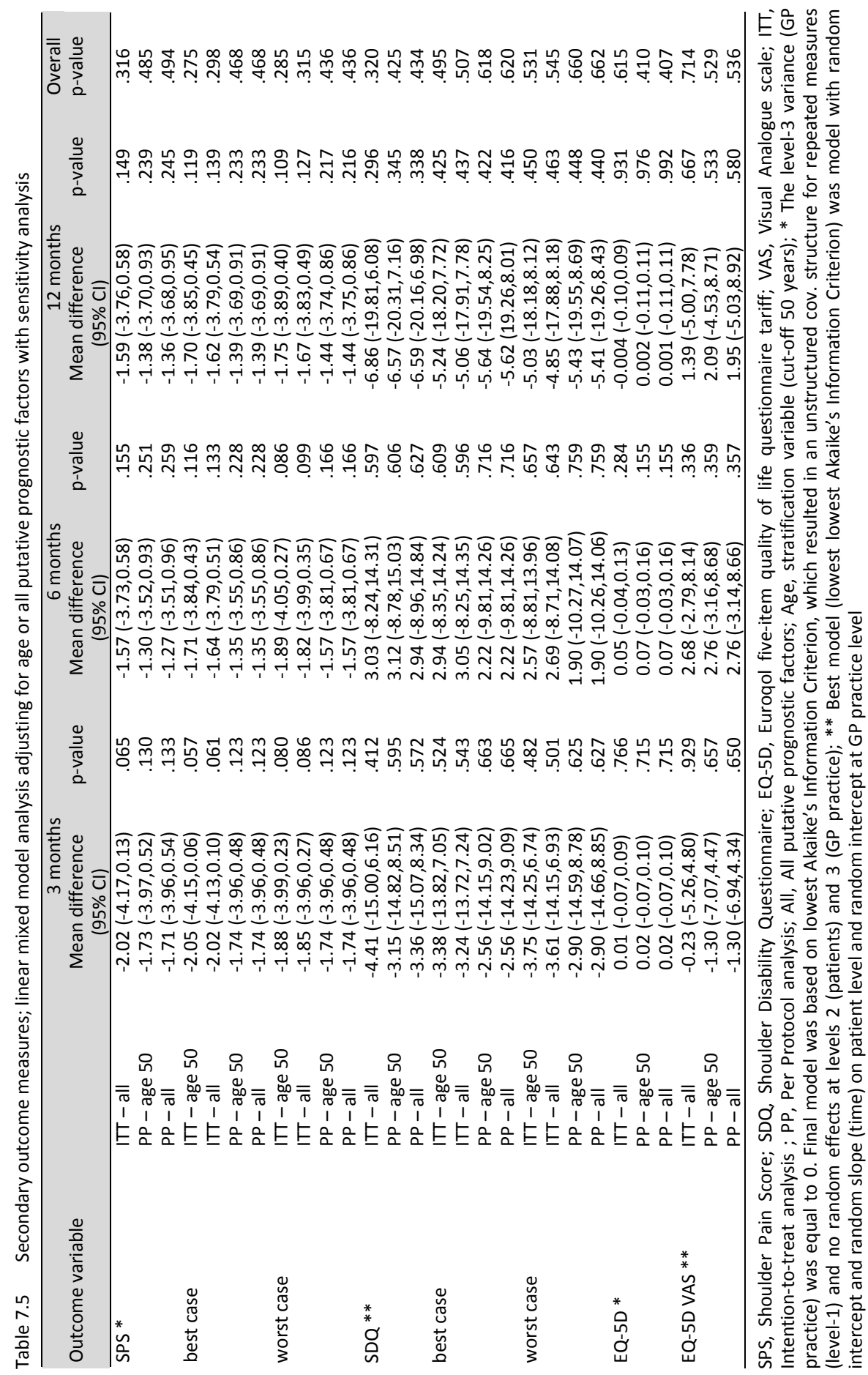




\section{Discussion}

\section{Implication of key findings}

We present evidence that ultrasound tailored treatment of patients with acute shoulder pain due to a subacromial disorder and without previous treatment, does not significantly improve patient perceived recovery after one year. Furthermore, this treatment does not influence healthcare use on study group level. However, the results do not give a conclusive answer to whether ultrasound tailored treatment improves outcome, as our trial was under-enrolled. These results question the value of applying ultrasound tailored treatment to the management of acute shoulder pain (duration of less than 3 months).

\section{Strengths and weaknesses}

To our knowledge this is the first primary care study investigating the effect of using ultrasound imaging in the management of patients with shoulder pain to target treatment to the specific underlying patho-anatomical disorders. This study has several strengths. Firstly, this study was developed as a pragmatic trial to inform clinicians, guideline developers and policy makers to choose wisely between options for care. ${ }^{30}$ Pragmatic trials measure effectiveness in routine clinical practice, and reflect variations between patients that occur in real clinical practice improving generalisability. ${ }^{40}$ Secondly, we filtered out patients with a favourable natural course (14\%) during the 2-week qualification period to prevent non-responders in the course of the trial. Thirdly, since blinding for ultrasound diagnosis in the usual care group had to be taken into account to prevent information bias, radiologists were not allowed to discuss their findings with patients. We evaluated this by asking patients one question in the questionnaire at 3 months; blinding of patients for the ultrasound diagnosis was violated only once. On the other hand, in five patients in the usual care group, the ultrasound diagnosis was disclosed to the GP by mistake. We incorporated these protocol violations in the per protocol analysis. Finally, our study seems representative as the recovery rate of $60 \%$ in the usual care group corresponds to recovery rate used in the sample size calculation, which was based on previous studies. ${ }^{3,4}$

Our study knows some limitations. Firstly, the recruiting GPs were asked to check eligibility of all patients with shoulder pain. Unfortunately we are unaware of the number of eligible patients as GPs rarely registered numbers and reasons for nonparticipation. However, this reflects the consultation process in general practice, where recruitment is rarely straightforward, but might influence the external validity of our findings. ${ }^{41}$ Secondly, although we carefully planned the recruitment aspects, and GPs 
beforehand positively responded to the feasibility, we did not reach the recruitment target. Patient recruitment for primary care based randomised trials is a known challenge. Literature shows that the demands of the study should be kept to a minimum for patients and clinicians and that dedicated research or support staff should be available to provide support and encouragement for the clinical staff. ${ }^{42}$ Hence, we decided to extend the recruitment period with an additional year and to adjust the inclusion procedure to further minimise the demands for recruiting GPs. The inclusion procedure was relocated from the GP practice to the Department of Radiology of the Orbis Medical Centre, providing two advantages. All GPs working in the region of the Orbis Medical Centre could refer patients for inclusion, and the GP's time load spent on study procedures decreased to a minimum. This adjusted inclusion procedure yielded an additional 42 patients. To correct for this change of inclusion procedure, we added recruitment strategy as a covariate to the mixed model analysis. Finally, instead of the estimated $80 \%$ recovery rate in the ultrasound tailored treatment group, we observed a recovery rate of $72.5 \%$ at 1 year, resulting in a non-statistically significant difference of $12.5 \%$. Recalculation of the between-group difference for a reduced sample size of 111 randomised patients indicated a recovery rate of approximately $23 \%$ was necessary to reach the level of significance. Very limited literature is available on the minimal clinically important difference for the Global Perceived Effect questionnaire. We choose this outcome measures as it provides the patient's perspective on the impact of disorder and treatment. ${ }^{43}$ One can argue that a difference of more than $10 \%$ is clinical important, but the population and context determines what is regarded clinically important. ${ }^{44}$ The observed difference of $12.5 \%$ would have been statistically significant if we had included 448 patients, far more than calculated and not feasible in this design.

\section{Comparison with other studies}

Previous studies in general practice focussed on the effectiveness of conservative treatments in patients with subacromial impingement syndrome or chronic complaints. For example, the effectiveness of two different injections (hyaluronic acid or corticosteroid), ${ }^{45}$ the effect of manual therapy, ${ }^{38,46}$ the effect of corticosteroid injection with exercise, ${ }^{47}$ and the comparison between conservative and surgical treatment in patients with subacromial impingement syndrome, ${ }^{48,49}$ or an exercise program in patients with chronic shoulder pain..$^{50,51}$ Instead of combining a diagnostic imaging test to inform GPs about underlying patho-anatomical disorders to tailor treatment to the observed underlying disorder, all these studies used the generic term subacromial impingement syndrome. We chose to label and define the shoulder disorders based on 
findings from physical examination followed by ultrasound imaging instead of solely findings from physical examination, as this does not lead to a precise diagnosis. ${ }^{10-12}$ This previously described lack in uniformity in the way shoulder disorders are labelled and defined hampers comparisons. ${ }^{52-54}$ Therefore, the content and effectiveness of this study cannot be compared to other randomised studies. Cadogan et al. performed a prospective diagnostic study in which clinical examination and ultrasound imaging were compared with results of diagnostic injections of local anaesthesia. ${ }^{25}$ They concluded that rotator cuff and bursa pathology were the most common findings, and that imaged pathology combined with clinical examination findings may inform GPs regarding treatment.

\section{Clinical implications}

The pragmatic design of our study enhances the generalisability of the findings. Our findings imply that GPs should refrain from ordering ultrasound imaging in patients with acute shoulder pain with no previous treatment. The current shoulder pain guidelines state that ultrasound imaging should be considered in cases with an abnormal course or therapy resistance, an advice that still holds.

GPs generally tend to ignore recommendations on additional imaging in guidelines as

ultrasound imaging is frequently applied in patients with acute shoulder pain. ${ }^{19,24,55}$ If GPs still consider ultrasound imaging, it might be used preferentially in patients aged 40 years and older, as over $90 \%$ of shoulder patients in this age group have a subacromial disorder, compared to a much lower prevalence in younger age groups. ${ }^{23}$

In patients with persistent pain, ultrasound imaging may yield a more specific diagnosis, provide a rationale for further treatment, and inform patients about the prognosis of their disorder. For daily practice it is important that ultrasound findings are considered in the clinical context, as asymptomatic findings may be detected. A situation that can pose a real challenge.

\section{Unanswered questions and future research}

Whether ultrasound imaging should be applied to the management of patients with acute shoulder pain depends on whether it improves patient outcomes, saves costs and affects diagnosis and treatment. The question whether ultrasound tailored treatment is cost-effective has not been answered. Although not calculated, health care costs in this study probably do not differ between groups as mean health care use is the same in both groups. However, it is suggested that episodes of shoulder pain generate additional costs for productivity losses due to sick leave. ${ }^{56}$ In an economic evaluation these costs should be incorporated. We showed that ultrasound imaging affects 
diagnosis, and most patients were diagnosed with calcific tendonitis, therefore, attention can be focused predominantly on this disorder. There is growing evidence that extracorporeal shock wave therapy and barbotage are effective treatment options in case of calcific tendonitis, and that corticosteroid injections can be harmful, ${ }^{57-60}$ which emphasises the importance of evaluating these treatment options in general practice.

\section{Conclusions}

Ultrasound tailored treatment in patients with acute shoulder pain without previous treatment in whom the GP suspected a subacromial disorder does not significantly improve recovery at 1 year. These results question the value of applying ultrasound tailored treatment to the early management of shoulder pain. From a clinical point of view, GPs should be informed that ultrasound imaging in patients with acute shoulder pain (duration of less than 3 months) does not provide additional information to improve patient outcome. 


\section{References}

1. Picavet HS, Schouten JS. Musculoskeletal pain in the Netherlands: prevalences, consequences and risk groups, the DMC(3)-study. Pain. 2003;102:167-178.

2. Greving K, Dorrestijn O, Winters JC, et al. Incidence, prevalence, and consultation rates of shoulder complaints in general practice. Scan J Rheumatol. 2012;41:150-155.

3. van der Windt DA, Koes BW, Boeke AJ, Devillé W, De Jong BA, Bouter LM. Shoulder disorders in general practice: prognostic indicators of outcome. Br J Gen Pract. 1996;46:519-523.

4. Winters JC, Sobel JS, Groenier KH, Arendzen JH, Meyboom-de Jong B. The long-term course of shoulder complaints: a prospective study in general practice. Rheumatology. 1999;38:160-163.

5. Kuijpers T, van der Windt DA, Boeke AJ, et al. Clinical prediction rules for the prognosis of shoulder pain in general practice. Pain. 2006;120:276-285.

6. Andersson IH. The course of non-malignant chronic pain: a 12-year follow-up of a cohort from the general population. Eur J Pain. 2004;8:47-53.

7. Macfarlane GJ, Hunt IM, Silman AJ. Predictors of chronic shoulder pain: a population based prospective study. J Rheumatol. 1998;25:1612-1615.

8. Luime JJ, Kuiper JI, Koes BW, Verhaar JAN, Miedema HS, Burdorf A. Work-related risk factors for the incidence and recurrence of shoulder and neck complaints among nursing-home and elderly-care workers. Scand J Work Environ Health. 2004;30:279-286.

9. Winters JC, van der Windt DAWM, Spinnewijn WEM, et al. Shoulder pain guideline of the Dutch College of General Practitioners (in Dutch). Huisarts Wet. 2008;51:555-565.

10. Dinnes J, Loveman E, Mclntyre L, Waugh N. The effectiveness of diagnostic tests for the assessment of shoulder pain due to soft tissue disorders: a systematic review. Health Technol Assess. 2003;7:1-166.

11. Hegedus EJ, Goode A, Campbell S, et al. Physical examination tests of the shoulder: a systematic review with meta-analysis of individual tests. Br J Sports Med. 2008;42:80-92.

12. Hermans J, Luime JJ, Meuffels DE, Reijman M, Simel DL, Bierma-Zeinstra SM. Does this patient with shoulder pain have rotator cuff disease?: The Rational Clinical Examination systematic review. JAMA. 2013;310:837-847.

13. NHS/NICE CKSb. Shoulder pain guideline. Available at: http://www.cks.nhs.uk/shoulder_pain.

14. Britt H, Miller GC, Knox S. Shoulder syndrome. In imaging orders by general practitioners in Australia 1999-2000. AlHW Cat. No. GEP 7. General Practice Series No. 7. Available at: www.aihw.gov.au/publications/index.cfm/title/6949 (2001).

15. Broadhurst NA, Gialamis A, McElroy HJ, Bielby JJ. How do Australian GPs manage shoulder dysfunction? Aust Fam Physician. 2004;33:861-864.

16. Buchbinder R, Staples MP, Shanahan EM, Roos JF. General practitioner management of shoulder pain in comparison with rheumatologist expectation of care and best evidence: an Australian national survey. PLoS One. 2013;8:e61243.

17. Loebenberg MI, Rosen JE, Ishak C, Jazrawi LM, Zuckerman JD. A survey of decision-making processes in the treatment of common shoulder ailments among primary care physicians. Bull Hosp Jt Dis. 2006;63: 137-144.

18. Masters S, O'Doherty L, Mitchell GK, Yelland M. Acute shoulder pain in primary care - an observational study. Aust Fam Physician. 2007;36:473-476.

19. Broadhurst N, Baghurst T, MacLaren S. Ultrasound imaging for shoulder pain in general practice. Aust Fam Physician. 2004;33:668-669.

20. Ottenheijm RPG, Jansen MJ, Staal JB, et al. Accuracy of diagnostic ultrasound in patients with suspected subacromial disorders: a systematic review and meta-analysis. Arch Phys Med Rehabil. 2010;91: 1616-1625.

21. Smith TO, Back T, Toms AP, Hing CB. Diagnostic accuracy of ultrasound for rotator cuff tears in adults: a systematic review and meta-analysis. Clin Radiol. 2011;66:1036-1048. 
22. Lenza M, Buchbinder R, Takwoingi $\mathrm{Y}$, Johnston RV, Hanchard NC, Faloppa F. Magnetic resonance imaging, magnetic resonance arthrography and ultrasonography for assessing rotator cuff tears in people with shoulder pain for whom surgery is being considered. Cochrane Database Syst Rev. 2013;9:CD009020.

23. Ottenheijm RP, Cals JW, Weijers R, Vanderdood K, de Bie RA, Dinant GJ. Ultrasound imaging for tailored treatment of patients with acute shoulder pain. Ann Fam Med. 2015;13:53-55.

24. Ottenheijm RP, van't Klooster IG, Starmans LM, et al. Ultrasound-diagnosed disorders in shoulder patients in daily general practice: a retrospective observational study. BMC Fam Pract. 2014;15:115.

25. Cadogan A, Laslett M, Hing WA, McNair PJ, Coates $\mathrm{MH}$. A prospective study of shoulder pain in primary care: prevalence of imaged pathology and response to guided diagnostic blocks. BMC Musculoskelet Disord. 2011;12:119.

26. Baring T, Emery RJH, Reilly P. Management of rotator cuff disease: specific treatment for specific disorders. Best Pract Res Clin Rheumatol. 2007;21:279-294.

27. Royal Dutch Society for Physical Therapy. Diagnosis and treatment of subacromial disorders (in Dutch). Available at: www.kngfrichtlijnen.nl (2010).

28. Hill JC, Whitehurst DG, Lewis M, et al. Comparison of stratified primary care management for low back pain with current best practice (STarT Back): a randomised controlled trial. Lancet. 2011;378: 1560-1571.

29. Ottenheijm RP, Joore MA, Walenkamp GH, et al. The Maastricht Ultrasound Shoulder pain Trial (MUST): ultrasound imaging as a diagnostic triage tool to improve management of patients with non-chronic shoulder pain in primary care. BMC Musculoskelet Disord. 2011;12:154.

30. Zwarenstein M, Treweek S, Gagnier JJ, et al. Improving the reporting of pragmatic trials: an extension of the CONSORT statement. BMJ. 2008;337:a2390.

31. Kamper SJ, Ostelo RW, Knol DL, Maher CG, de Vet HC, M.J. H. Global Perceived Effect scales provided reliable assessments of health transition in people with musculoskeletal disorders, but ratings are strongly influenced by current status. J Clin Epidemiol. 2010;63:760-766.

32. Winters JC, Sobel JS, Groenier KH, Arendzen JH, Meyboom-De Jong B. A shoulder pain score: a comprehensive questionnaire for assessing pain in patients with shoulder complaints. Scand J Rehabil Med. 1996;28:163-167.

33. de Winter AF, van der Heijden GJ, Scholten RJ, van der Windt DA, Bouter LM. The Shoulder Disability Questionnaire differentiated well between high and low disability levels in patients in primary care, in a cross-sectional study. J Clin Epidemiol. 2007;60:1156-1163.

34. Brooks R. EuroQol: the current state of play. Health Policy. 1996;37:53-72.

35. Picavet HS, Hoeymans N. Health related quality of life in multiple musculoskeletal diseases: SF-36 and EQ-5D in the DMC3 study. Ann Rheum Dis. 2004;63:723-729.

36. Dolan P. Modeling valuations for EuroQol health states. Medical care. 1997;35:1095-1108.

37. Sullivan MJ, Bishop SR, Pivik J. The pain catastrophizing scale: development and validation. Psycholog Assess. 1995; 7:524-532.

38. Bergman GJ, Winters JC, Groenier KH, et al. Manipulative therapy in addition to usual medical care for patients with shoulder dysfunction and pain: a randomized, controlled trial. Ann Intern Med. 2004;141: 432-439.

39. Geraets JJ, Goossens ME, de Bruijn CP, et al. Cost-effectiveness of a graded exercise therapy program for patients with chronic shoulder complaints. Int J Technol Assess Health Care. 2006;22:76-83.

40. Roland M, Torgerson DJ. What are pragmatic trials? BMJ. Jan 24 1998;316:285.

41. Jenkinson CE, Winder RE, Sugg HV, et al. Why do GPs exclude patients from participating in research? An exploration of adherence to and divergence from trial criteria. Fam Pract. Jun 2014;31:364-370.

42. Ross S, Grant A, Counsell C, Gillespie W, Russell I, Prescott R. Barriers to participation in randomised controlled trials: a systematic review. J Clin Epidemiol. 1999;52:1143-1156.

43. Revicki D, Hays RD, Cella D, Sloan J. Recommended methods for determining responsiveness and minimally important differences for patient-reported outcomes. J Clin Epidemiol. 2008;61:102-109.

44. Beaton DE, Boers M, Wells GA. Many faces of the minimal clinically important difference (MCID): a literature review and directions for future research. Curr Opin Rheumatol. 2002;14:109-114. 
45. Penning LI, de Bie RA, Walenkamp GH. The effectiveness of injections of hyaluronic acid or corticosteroid in patients with subacromial impingement: a three-arm randomised controlled trial. J Bone Joint Surg. 2012;94:1246-1252.

46. Kromer TO, de Bie RA, Bastiaenen $\mathrm{CH}$. Effectiveness of physiotherapy and costs in patients with clinical signs of shoulder impingement syndrome: One-year follow-up of a randomized controlled trial. J Rehabil Med. 2014;46:1029-1036.

47. Crawshaw DP, Helliwell PS, Hensor EM, Hay EM, Aldous SJ, Conaghan PG. Exercise therapy after corticosteroid injection for moderate to severe shoulder pain: large pragmatic randomised trial. BMJ. 2010;340:c3037.

48. Dorrestijn O, Stevens M, Winters JC, van der Meer K, Diercks RL. Conservative or surgical treatment for subacromial impingement syndrome? A systematic review. J Shoulder Elbow Surg. 2009;18:652-660.

49. Kromer TO, Tautenhahn UG, de Bie RA, Staal JB, Bastiaenen CH. Effects of physiotherapy in patients with shoulder impingement syndrome: a systematic review of the literature. J Rehabil Med. 2009;41;870-880.

50. Geraets JJ, Goossens ME, de Groot IJ, et al. Effectiveness of a graded exercise therapy program for patients with chronic shoulder complaints. Aust J Physiother. 2005;51:87-94.

51. de Bruijn C, de Bie R, Geraets J, et al. Effect of an education and activation programme on functional limitations and patient-perceived recovery in acute and sub-acute shoulder complaints - a randomised clinical trial. BMC Musculoskelet Disord. 2007;8:112.

52. Green S, Buchbinder R, Glazier R, Forbes A. Systematic review of randomised controlled trials of interventions for painful shoulder: selection criteria, outcome assessment, and efficacy. BMJ. 1998;316:354-360.

53. Schellingerhout JM, Verhagen AP, Thomas S, Koes BW. Lack of uniformity in diagnostic labeling of shoulder pain: time for a different approach. Man Ther. 2008;13:478-483.

54. de Witte PB, de Groot JH, van Zwet EW, et al. Communication breakdown: clinicians disagree on subacromial impingement. Med Biol Eng Comput. 2014;52:221-231.

55. Ottenheijm RP, Hesselmans NJ, Kemper A, et al. GPs' perspectives on the diagnostic work-up in patients with shoulder pain: a qualitative study. J Eval Clin Pract. 2014;20:239-245.

56. Kuijpers T, van Tulder MW, van der Heijden GJMG, Bouter LM, van der Windt DIAWM. Costs of shoulder pain in primary care consulters: a prospective cohort study in The Netherlands. BMC Musculoskelet Disord. 2006;7:83.

57. Bannuru RR, Flavin NE, Vaysbrot E, Harvey W, McAlindon T. High-energy extracorporeal shock-wave therapy for treating chronic calcific tendinitis of the shoulder: a systematic review. Ann Intern Med. 2014;160:542-549.

58. de Witte PB, Selten JW, Navas A, et al. Calcific tendinitis of the rotator cuff: a randomized controlled trial of ultrasound-guided needling and lavage versus subacromial corticosteroids. Am J Sports Med. 2013;41:1665-1673.

59. Dean BJ, Franklin SL, Murphy RJ, Javaid MK, Carr AJ. Glucocorticoids induce specific ion-channel mediated toxicity in human rotator cuff tendon: a mechanism underpinning the ultimately deleterious effect of steroid injection in tendinopathy? Br J Sports Med. 2014;48:1620-1626.

60. Verstraelen FU, In den Kleef NJ, Jansen L, Morrenhof JW. High-energy versus low-energy extracorporeal shock wave therapy for calcifying tendinitis of the shoulder: which is superior? A meta-analysis. Clin Orthop Rel Res. 2014;472:2816-2825. 


\section{Chapter 8}

General discussion 
Chapter 8 


\section{General discussion}

This thesis investigates the potential role of ultrasound imaging for improved management of acute shoulder pain due to subacromial disorders in general practice patients. This Chapter is divided into two parts: in Part 1, the main methodological challenges are addressed; while in Part 2, the main findings of the studies and their clinical implications are discussed. Finally, directions for future research are presented.

* Part 1: Methodological reflections

* Part 2: Discussion of main findings, and their implications for clinical practice and future research 


\section{Part 1}

\section{Methodological reflections}

In our studies we investigated a variety of research questions using a range of quantitative and qualitative research methodologies: a systematic review with metaanalysis of diagnostic test accuracy (Chapter 2), an explorative qualitative design with semi-structured interviews (Chapter 3), two observational studies (Chapters 4 and 6), and a pragmatic, randomised controlled multicentre trial (Chapters 5 and 7). The specific methodological strengths and limitations are discussed within their respective Chapter. This section reflects on how we dealt with one of the major challenges in primary care research: prospectively enrolling patients with acute conditions into clinical trials.

\section{Patient recruitment in primary care trials: what's known?}

A major problem to the completion of trials in primary care is recruiting adequate numbers of patients. This phenomenon, known as Lasagna's Law, occurs when research investigators overestimate the pool of eligible study participants, and has been previously described for Dutch primary care and shoulder pain. ${ }^{1,2}$ Within the planned recruitment period, only around $50 \%$ of the studies in Dutch primary care succeed in recruiting target patient numbers, and extension of the recruitment period is often applied to remedy those studies that do not achieve their targets. ${ }^{2}$

It is recommended that recruitment aspects of a trial are carefully planned and preferably piloted to avoid poor recruitment. ${ }^{3}$ For example, approaching GPs through regional GP groups or existing networks is more successful than approaching them individually. ${ }^{2}$ In addition, the following guidance should be taken into consideration. Firstly, the study should address an important research question, and the study protocol and data collection should be as straightforward as possible. ${ }^{3}$ Secondly, studies that focus on prevalent cases are more successful than those requiring incident cases, while studies requiring high GP involvement are less successful in recruiting adequate numbers of patients. ${ }^{2}$ In addition, the demands on GPs and patients should be kept to a minimum, and patients should be supported in their decision to take part in a trial. ${ }^{3}$ Therefore, dedicated research and support staff play a critical role in the provision of information to GPs and patients, and obtaining patient consent. ${ }^{3}$ This is particularly important when GPs are faced with difficult decisions or ethical dilemmas 
when inviting patients to participate in a trial, or in cases where the GP views the research process as a burden to patients and practice staff. ${ }^{4}$

\section{How did we manage patient recruitment?}

As outlined in Chapter 5, we originally aimed to recruit 226 patients within a period of two years, which would have resulted in 180 randomised patients. ${ }^{5}$ However, this recruitment period of two years had to be extended by one year. Even with this extension, we managed to enroll only 129 patients, of whom 111 were randomised. Given we were unable to recruit the target number of patients, we concluded that our trial was also affected by Lasagna's Law. In order to understand why this occurred, one must understand and evaluate both the GP and the patient perspectives on our recruitment process. Below we provide some insight into the way we handled this situation, and present some lessons learned.

\section{How did we recruit participating GPs?}

We calculated that 20 GPs were needed to achieve our target number of patients, and had no issues in their recruitment. The study was conducted in the Westelijke Mijnstreek, a region in the south of the Netherlands, with approximately 80 GPs working in 48 practices. Medical Coordination Centre Omnes (Omnes) supported our study from the beginning, as the study results would be of great interest to them. Omnes is cooperation between regional GPs and the hospital Orbis Medical Centre (OMC), which provides all diagnostic tests ordered by GPs.

After a single recruitment phase, we reached our target number of 21 GPs, working in 11 practices. Subsequently, the participating GPs and their practice nurses were trained in the management of shoulder pain patients and in conducting the research protocol. ${ }^{5,6}$ The GPs received a laminated card to quickly check patient eligibility during consultations. In addition, it was agreed that the practice nurses should alert the GP before the start of the consultation if the case had potential relevance to our study. GPs would receive a reimbursement of $€ 75$ per patient.

\section{How did we tackle patient recruitment?}

The patients that were to be included in our study had to be incident cases only. Selecting prevalent cases by searching the electronic patient records was not a viable option, as patients were only eligible if they consulted their GP within 3 months of onset of pain and if they were untreated previously. However, during the recruitment period it became clear that the target number of patients would be difficult to achieve. 
Therefore, we employed three strategies to facilitate greater patient inclusion: first, we conducted an additional procedure to recruit more GPs (I); second, we requested that participating physiotherapists refer potentially eligible patients (II); and third, we relocated the inclusion procedure from the GP practices to the Department of Radiology of OMC (III). This relocation was combined with the extension of the recruitment period by an additional year.

\section{I) Additional GP recruitment}

Several participating GPs from the original recruitment group of 20 noted that several patients with shoulder pain did not meet the two most important inclusion criteria, being 1) duration of pain no longer than three months; and 2) no previous treatment. As these patients could not participate in our study, our recruitment numbers were lower than expected. As we did not want to compromise on these criteria, we opted to recruit additional GPs in the Westelijke Mijnstreek. This yielded seven additional GPs, working in six practices, however this strategy later showed that it did not adequately increase the number of patients recruited to our study.

\section{II) Identification of eligible patients by physiotherapists}

We trained 23 physiotherapy practices in the Westelijke Mijnstreek to apply an evidence-based approach for subacromial disorders. ${ }^{7}$ From the commencement of the study, they treated our study patients only upon referral by the GP. Given our challenges in patient recruitment, we subsequently invited these physiotherapists to identify potential eligible patients for our study and refer them to a participating GP for consultation and study inclusion. Ten practices responded positively, after which they were trained in the study aspects. Most importantly, physiotherapists were instructed to refer potential patients to their GP after screening for an additional eligibility check, and from that point onward the physiotherapy management had to be suspended. Unfortunately, this strategy yielded no additional patients. The reasons for this failure were not evaluated.

\section{III) Relocation of the inclusion procedure}

As a final strategy to recruit patients, we relocated the inclusion procedure from the GP practices to the Department of Radiology at the OMC. We also adapted the application form released by Omnes for which GPs have to complete when ordering ultrasound exams. In Chapter 4, we described that in the Westelijke Mijnstreek GPs order approximately 2400 ultrasound examinations of the shoulder annually. In cooperation with Omnes we developed an evidence-based flowchart to specify when a GP should 
advise ultrasound imaging to shoulder patients. Our study was incorporated in this flowchart, which formed part of the new application form released by Omnes. This flowchart enabled GPs to inform eligible patients about our study, and to refer them to the Department of Radiology of the OMC for more information and study inclusion. This procedure was explained to the GPs, and written information was provided. The staff of the Department of Radiology were also trained in the research protocol.

The relocation had two main advantages. Firstly, as GP practices were no longer considered a study centre by the Medical Ethical Committee, all GPs working in the Westelijke Mijnstreek were able to refer patients for inclusion. Secondly, time spent by GPs on patient recruitment decreased to a minimum. This inclusion procedure was implemented two years after the onset of our study and yielded 42 additional patients, bringing the total number of included patients to 129. As this recruitment strategy might have induced a form of selection bias, it was added as a covariate to the mixed model analysis (Chapter 7).

\section{Recruitment closure}

Over time, it became clear that the target number of patients set out in our study protocol could not be achieved within three years of recruitment. We therefore decided to close the recruitment period on the basis of the following two considerations. Firstly, the current inclusion rate for this period indicated an additional one and a half years would have been required to reach the target number of 180 randomised patients. Further extension of the study period to achieve this target was no longer feasible within the allotted time frame of this thesis. Secondly, the original sample size was calculated on a between-group difference in recovery rate of $20 \%$. However, recalculation of the between-group difference for a reduced sample size of 110 randomised patients, which we believed we could attain after three years of recruitment, indicated a recovery rate of approximately $23 \%$ was necessary to reach the level of significance. We considered this three-percent difference in recovery rate to be small enough that closure of the recruitment period at three years was the best option.

\section{Lessons learned}

Recruiting GPs by approaching them through a regional GP organisation was a successful strategy. This strategy has been described by Van der Wouden et al, who stated that recruiting GPs by approaching them this way is more successful than approaching them individually. ${ }^{2}$ Many GPs recognised that the study addressed an 
important research question, which has been described as a facilitator for participation. ${ }^{3}$ This recognition undoubtedly contributed to the success of this strategy. Unfortunately, our strategies to increase recruitment rate were unsuccessful, and the recruitment process was more cumbersome than anticipated. Several explanations might be given for the failure to recruit our planned number of patients. Firstly, the incidence of untreated patients with acute shoulder pain was lower than we had expected. Even though the disorder is frequently encountered, a recent study showed that the majority of shoulder patients wait more than three months before seeking help from a GP, or have already sought help from a physiotherapist before seeing a GP. ${ }^{8}$ These patients were not eligible for our study. A pilot study might have pointed out earlier to us that several patients would therefore not meet the inclusion criteria.

Secondly, we recruited only incident cases during GP consultations, whereas the additional inclusion of prevalent cases might have yielded a higher number of patients. Prevalent cases can easily be identified by searching the electronic patient files, while incident cases require a greater effort by GPs during consultation in order to recruit eligible patients to the study. ${ }^{2}$

Thirdly, given these challenges, it is understood that recruiting incident cases during consultations places a burden on GPs. Despite the fact that the demands on GPs were kept to a minimum and some tasks could be delegated to practice nurses, the workload was nevertheless a barrier for the GPs. To remedy this problem, we relocated the inclusion procedure from the GP practice to the Department of Radiology, where dedicated staff were trained. This action could have been taken earlier in the study.

Fourthly, a relevant hypothesis formulated by Van Bokhoven et al. may apply to our study: before GPs realise that a patient is eligible, they may have already discussed successive management with the patient, which may consequently deter study participation. ${ }^{9}$ For example, the GP may discuss the benefits of ultrasound imaging with the patient, in which case the patient may then be reluctant to participate in the study, not wanting to be allocated to the control group. ${ }^{4}$ Moreover, several GPs founded it difficult to explain to patients that they had a $50 \%$ chance of non-disclosure of the ultrasound diagnosis. Non-disclosure can lead to worry caused by uncertainty, ${ }^{3}$ and prevention of this unnecessary burden is a known protective role of GPs. ${ }^{4}$ This could have been prevented by randomising at the GP level instead of at the patient level, however, a larger sample size would have been required. ${ }^{10}$ Given that recruitment of GPs was not a barrier in our study, this was an option that could have been pursued. These lessons were valuable and have potential implications for similar trials in general practice. 


\section{Part 2}

\section{Discussion of main findings, and their implications for clinical practice and future research}

The primary objective of this thesis is to investigate the effects of tailoring treatment to an ultrasound diagnosis on clinical recovery, compared to the current standard of care for patients with acute shoulder pain (pain less than 3 months), who present in general practice. The findings in this thesis suggest that management of patients with shoulder pain can be improved. In this section, the main findings of our studies and their clinical implications will be discussed. Finally, directions for future research will be presented.

\section{Discussion of main findings}

In general practice, establishing a specific diagnosis in patients with shoulder pain is not possible on the basis of medical history and physical examination alone. In Chapter 2, the results of our systematic review show that ultrasound imaging is accurate in diagnosing subacromial disorders in patients for whom conservative treatment fails. ${ }^{11}$ This implies that ultrasound imaging enables GPs to establish a more specific diagnosis, as these disorders are present earlier in the course. As discussed in Chapter 3, GPs prefer to establish a specific diagnosis in patients with shoulder pain, whereas this is not advised in the shoulder pain guidelines for GPs. GPs use several classifications to aid the diagnosis of shoulder pain, as they experience the diagnostic process as complex. To deal with this complexity, GPs apply different referral strategies including referral to orthopaedic surgeons and ultrasound imaging. ${ }^{12}$ This latter strategy is discussed in Chapter 4, where it is shown that GPs frequently apply for ultrasound imaging in patients with shoulder pain, and subsequently the full spectrum of subacromial disorders is observed. ${ }^{13}$ In Chapter 6 , we show that calcific tendonitis is the most frequently diagnosed disorder in patients with acute shoulder pain $(50 \%)$ and that $43 \%$ of the patients have multiple disorders. We also observed that subacromial disorders are strongly related to age and seen most often in patients aged 40 or older. ${ }^{14}$ This suggests that ultrasound imaging allows more tailored treatment than currently applied. In Chapter 7, we present evidence that ultrasound tailored treatment of patients with acute shoulder pain due to a subacromial disorder and without previous treatment, does not significantly improve patient perceived recovery after one year. However, it does not give a conclusive answer to whether ultrasound tailored 
treatment improves the clinical outcome, as our trial was under-enrolled. Furthermore, this treatment does not influence healthcare use at the study group level.

Our results question the value of applying ultrasound tailored treatment to the management of acute shoulder pain in general practice. Despite our trial being underenrolled, discussed in Chapter 7 and Part 1 of the General Discussion, several other explanations for this lack of effect can be given. At first, there is no difference in healthcare use between the study groups in our trial (I), and a more comprehensive approach is necessary to tailor treatment (II).

\section{I) No difference in health care use}

In the two study groups, patients were prescribed medication, administered corticosteroid injections, and referred to physiotherapy or secondary care. In contrast to what we expected, we did not observe significant differences between the two study groups with regards to the proportion of patients who received these treatments (Chapter 7). This might be explained by the discrepancy between the proportions of expected and actually observed subacromial disorders, and choices made while developing our treatment protocol.

In the design phase of our trial, there was no data available from primary care regarding the prevalence of specific shoulder disorders. Based on the available literature at that point and our clinical experience, we expected that tendinopathy would be the most prevalent observed disorder, for which physiotherapy was first-line treatment in our protocol. However, calcific tendonitis was by far the most frequently observed disorder in the ultrasound tailored treatment group (46\% vs $29 \%$ for tendinopathy; Chapter 7). Our treatment protocol considered that corticosteroid injections were first-line treatment for this disorder (Chapter 5$).^{5}$ As a result, corticosteroid injections were administered to $39 \%$ of patients allocated to the ultrasound tailored treatment group, whereas this proportion was $31 \%$ in the usual care group; a non-significant difference (Chapter 7). The proportions observed are in line with those observed in usual care in a Dutch observational study in primary care. ${ }^{8}$ This indicates that corticosteroid injections are also frequently applied when treatment is tailored to the underlying disorder. However, there is growing evidence that corticosteroid injections may have harmful effects on tendons, ${ }^{15}$ which may negatively influence prognosis. Other treatments are available for calcific tendonitis, for example, extracorporeal shock wave therapy (ESWT), barbotage (aspiration and needling of the calcium deposit) and surgery. ${ }^{16-18}$ However, in the design phase of our trial, little evidence was available for these treatments. Apart from referral to an orthopaedic surgeon, these options were not explicitly included in our treatment protocol. ESWT and barbotage were not applied in our trial. 


\section{II) A more comprehensive approach}

In our study, we evaluated treatment strategies used by GPs that specifically targeted subacromial disorders, as detected by ultrasound imaging. However, the pathological mechanisms of subacromial disorders are incompletely understood, and more complicated than first thought. In view of growing insight in the etiology of these disorders, our approach might not have been comprehensive enough.

Subacromial disorders are now considered to be multifactorial conditions, with contributing intrinsic tendon factors such as the pathological process of tendinopathy, and extrinsic factors such as altered muscle dynamics, which are both poorly understood. ${ }^{19-21}$ The relationship between the disorders and perceived pain is also not fully understood. ${ }^{22}$

In tendinopathy, Cook and Purdam propose and provide evidence for a continuum of pathology, in which there are three distinguishable stages. This novel model is based on histopathological, clinical and imaging findings. ${ }^{19}$ Oliva et al. describe a pathway that may lead to calcium depositions in tendons, and advocate the term calcific tendinopathy instead of calcific tendonitis as it underlines the lack of a clear pathogenesis. ${ }^{23} \mathrm{~A}$ key feature of these models is that the pathology and response to treatment are different depending on the stage of pathology. This suggests that a single intervention, for example, the administration of a corticosteroid injection or NSAIDs, may be effective in one stage while deleterious in another. Regarding altered muscle dynamics, rotator cuff weakness and scapular dysfunction should be considered, as there is growing evidence that scapular dysfunction plays an important role in the development of subacromial disorders. ${ }^{24,25}$ However, examination of scapula function and its treatment is not currently mentioned in the shoulder pain guidelines for GPs. ${ }^{6}$ It has become clear that treatment focussing only on the observed ultrasound disorders may not have been comprehensive enough, and that applying a single intervention to all presentations of subacromial disorder is unlikely to be effective in every patient. Treatment should also address the modifiable factors contributing to the development and maintenance of the disorder. Therefore, we conclude that a highly tailored treatment approach, greater than what was applied in our trial, is necessary for improved patient outcomes.

\section{Implications for clinical practice}

The most optimal treatment strategy for subacromial disorders has not been determined from this thesis. However, the results obtained are nevertheless relevant 
and helpful in the management of patients with shoulder pain. Here, the implications for daily practice and guidelines development are discussed.

\section{Daily practice}

The current shoulder pain guidelines state that ultrasound imaging should be considered in cases with an abnormal course or therapy resistance. ${ }^{6}$ We provide two further suggestions that may be incorporated with these guidelines. Firstly, ultrasound imaging can be used in patients in need of reassurance (Chapter 3 ). Secondly, it could be used preferentially in patients aged 40 years or older, as over $90 \%$ of shoulder pain patients in this age group have a subacromial disorder, compared to a much lower prevalence in younger age groups (Chapter 6$).{ }^{14}$

The role of ultrasound imaging in cases of therapy resistance provides promising applications as well as challenges. In patients with persistent pain, ultrasound imaging may yield a more specific diagnosis, provide a rationale for further treatment, and inform patients about the prognosis of their disorder. For daily practice, it is important that ultrasound findings are considered in the clinical context, as asymptomatic findings may be detected. For example, in patients aged 40 years and older presenting with shoulder pain, calcific tendonitis is present in 6 out of 10 patients (Chapter 6$).{ }^{14}$ It is possible that the observed calcium deposits are asymptomatic. Therefore, ultrasound reports should contain information about the size and location of the calcium deposits as well as the morphologic shape of the deposit and the presence of neovascularity as this might predict symptomatology. ${ }^{26,27}$ This additional information enables GPs to inform patients about the natural course, which is considered to be self-limiting with recurrent painful episodes. If painful episodes are bothersome, treatments such as ESWT and barbotage should be considered rather than corticosteroid injections which may harm tendons. ${ }^{15-18}$ While these two treatments are promising, their use in general practice needs to be determined. Therefore, treatment choice should be guided by shared decision-making based on information as to both the effectiveness and adverse events of each treatment.

An evaluation of both the advantages and disadvantages of ultrasound imaging should be taken into consideration before use. When performing diagnostic imaging tests, there is always a risk of spurious findings and the related treatment of asymptomatic disorders. In $79 \%$ of the patients of our study, at least one disorder was present, while $43 \%$ of patients had multiple disorders (Chapter 6 ). ${ }^{14}$ This may lead to the unnecessary treatment of asymptomatic disorders, or treatment targeted at the wrong disorder when multiple disorders are present. 
Our study showed that approximately $60 \%$ of GP patients are referred to physiotherapy, thus the collaboration between GPs and physiotherapists is important for positive patient outcomes (Chapter 7). Physiotherapists are advised to apply the guidelines "Evidence Statement for Subacromial Pain" released by the Royal Dutch Society for Physical Therapy. ${ }^{7}$ One recommendation from these guidelines is to manage modifiable factors contributing to the development and maintenance of shoulder pain, for example, scapular dysfunction. Collaboration enables applying more than one intervention. Therefore, it is advisable that collaborating GPs and physiotherapists arrange an optimal treatment strategy.

\section{Guideline developers}

In order to inform patients about the natural course of a specific disorder and evidencebased treatments, GPs require more information than is currently available in the shoulder pain guidelines, released in 2008 by the Dutch College of General Practitioners. $^{6}$ These guidelines lack information about the natural course and treatments of the specific subacromial disorders. We recommend that when updating the guidelines, this omission is remedied, especially given that ultrasound imaging is frequently ordered by GPs (Chapters 3 and 4). ${ }^{12,13}$

We also recommend the introduction of a clear and unequivocal classification of shoulder pain (Chapter 3). ${ }^{12}$ Uniform classifications are essential for the timely diagnosis and treatment of shoulder pain conditions by various healthcare professionals such as GPs, physiotherapists, orthopedic surgeons and rheumatologists. Despite the shoulder pain guidelines advising GPs to classify patients based on pain and physical examination findings, GPs use a variety of classifications. This lack of uniformity is not limited to general practice, but also exists among physiotherapists, orthopedic surgeons and researchers. ${ }^{28,29}$ In 2012, the Netherlands Orthopaedic Association introduced the term "Subacromial pain syndrome (SAPS)" in the eponymous "Guideline for diagnosis and treatment of subacromial pain syndrome". ${ }^{30}$ We therefore recommend the use of the term SAPS in the shoulder pain guidelines of the Dutch College of General Practitioners, especially considering the guidelines for physiotherapists, released by the Royal Dutch Society for Physical Therapy, are known as "Evidence Statement for Subacromial pain" ${ }^{6,7}$ In cases where the specific disorders are known, SAPS can be replaced by this specific disorder. 


\section{Implications for future research}

This thesis shows that subgroups based on specific disorders exist among the heterogeneous population of patients with shoulder pain in general practice (Chapters 4 and 6). ${ }^{13,14}$ This challenges GPs to tailor treatment to specific disorders, however, applying a single intervention to all presentations of a subacromial disorder is unlikely to be effective for every patient.

The decision to use ultrasound imaging for the management of patients with acute shoulder pain depends on whether it affects diagnosis and treatment, improves patient outcomes and is cost-effective. Here, some suggestions for future research that may optimise the management of patients with shoulder pain in general practice, are presented.

Firstly, future research should focus on understanding the etiology of specific subacromial disorders in order to develop an effective, tailored treatment. Research in general practice should focus on the specific disorders rather than a heterogeneous group of patients with a variety of underlying disorders. We showed that most patients were diagnosed with calcific tendonitis or tendinopathy (Chapters 4 and 6), therefore, attention should be focused predominantly on these two disorders. ${ }^{13,14}$ Further, there is growing evidence that ESWT and barbotage are effective treatment options for calcific tendonitis, and that corticosteroid injections can be harmful, ${ }^{15-18}$ which emphasises the importance of evaluating treatment options for each disorder.

Secondly, the duration of the follow-up period is important, as recurrence rates have been shown to vary from $31 \%$ after 12 months to $85 \%$ after 12 years. ${ }^{31-33}$ The duration of the follow-up period in our randomised controlled trial was set at 12 months. Studies with a longer follow-up period are required to gain insight into the long term patient outcomes and costs.

Thirdly, cost-effectiveness studies are required, as it has been suggested that prolonged and recurrent episodes of shoulder pain generate additional healthcare costs and productivity losses due to sick leave. ${ }^{34}$ Therefore, future research should also incorporate an economic evaluation in order to inform all of the stakeholders (physicians, guideline developers and policy makers). We anticipate an economic evaluation of our trial to follow, in which analyses will be performed from a societal perspective, meaning that healthcare costs, patient and family costs, as well as productivity losses will be included.

Finally, as improved prognosis is the ultimate goal of managing patients with shoulder pain, research into prognosis is equally important as research into diagnosis and intervention. ${ }^{35}$ For example, in general practice patients with low back pain, a stratified management approach in which prognostic screening and treatment targeting were 
combined, saw improved patient outcomes. ${ }^{36}$ Through targeting treatments according to the underlying disorders or risk characteristics shared by subgroups of patients, known as stratified medicine, patients can be grouped according to the particular treatment that will provide the greatest clinical benefit or least harm. ${ }^{37}$ Fortunately, ultrasound imaging allows for stratified medicine; however, the prognoses of the different subgroups are unknown. Therefore, future research to establish prognostic factors may contribute to more effective tailored treatment and improved patient outcomes. 


\section{References}

1. Geraets JJ, de Groot IJ, Goossens ME, et al. Comparison of two recruitment strategies for patients with chronic shoulder complaints. Br J Gen Pract. 2006;56:127-133.

2. van der Wouden JC, Blankenstein AH, Huibers MJ, van der Windt DA, Stalman WA, Verhagen AP. Survey among 78 studies showed that Lasagna's law holds in Dutch primary care research. J Clin Epidemiol. 2007;60:819-824.

3. Ross S, Grant A, Counsell C, Gillespie W, Russell I, Prescott R. Barriers to participation in randomised controlled trials: a systematic review. J Clin Epidemiol. 1999;52:1143-1156.

4. Jenkinson CE, Winder RE, Sugg HV, et al. Why do GPs exclude patients from participating in research? An exploration of adherence to and divergence from trial criteria. Fam Pract. 2014;31:364-370.

5. Ottenheijm RP, Joore MA, Walenkamp GH, et al. The Maastricht Ultrasound Shoulder pain Trial (MUST): ultrasound imaging as a diagnostic triage tool to improve management of patients with non-chronic shoulder pain in primary care. BMC Musculoskelet Disord. 2011;12:154.

6. Winters JC, van der Windt DAWM, Spinnewijn WEM, et al. Shoulder pain guidelines of the Dutch College of General Practitioners (in Dutch). Huisarts Wet. 2008;51:555-565.

7. Royal Dutch Society for Physical Therapy. Diagnosis and treatment of subacromial disorders (in Dutch). Available at: www.kngfrichtlijnen.nl. 2011.

8. Kooijman M, Swinkels I, van Dijk C, de Bakker D, Veenhof C. Patients with shoulder syndromes in general and physiotherapy practice: an observational study. BMC Musculoskelet Disord. 2013;14:128.

9. van Bokhoven MA, Koch $H$, van der Weijden T, Dinant GJ. Special methodological challenges when studying the diagnosis of unexplained complaints in primary care. J Clin Epidemiol. 2008;61:318-322.

10. Smeeth L, Ng ES. Intraclass correlation coefficients for cluster randomized trials in primary care: data from the MRC Trial of the Assessment and Management of Older People in the Community. Control Clin Trials. 2002;23:409-421.

11. Ottenheijm RPG, Jansen MJ, Staal JB, et al. Accuracy of diagnostic ultrasound in patients with suspected subacromial disorders: a systematic review and meta-analysis. Arch Phys Med Rehabil. 2010;91: 1616-1625.

12. Ottenheijm RP, Hesselmans NJ, Kemper A, et al. GPs' perspectives on the diagnostic work-up in patients with shoulder pain: a qualitative study. J Eval Clin Pract. 2014;20:239-245.

13. Ottenheijm RP, van't Klooster IG, Starmans LM, et al. cus. BMC Fam Pract. 2014;15:115.

14. Ottenheijm RP, Cals JW, Weijers R, Vanderdood K, de Bie RA, Dinant GJ. Ultrasound imaging for tailored treatment of patients with acute shoulder pain. Ann Fam Med. 2015;13:53-55.

15. Dean BJ, Franklin SL, Murphy RJ, Javaid MK, Carr AJ. Glucocorticoids induce specific ion-channelmediated toxicity in human rotator cuff tendon: a mechanism underpinning the ultimately deleterious effect of steroid injection in tendinopathy? Br J Sports Med. 2014;48:1620-1626.

16. Bannuru RR, Flavin NE, Vaysbrot E, Harvey W, McAlindon T. High-energy extracorporeal shock-wave therapy for treating chronic calcific tendinitis of the shoulder: a systematic review. Ann Intern Med. 2014;160:542-549.

17. de Witte PB, Selten JW, Navas A, et al. Calcific tendinitis of the rotator cuff: a randomized controlled trial of ultrasound-guided needling and lavage versus subacromial corticosteroids. Am J Sports Med. 2013;41:1665-1673.

18. Verstraelen FU, In den Kleef NJ, Jansen L, Morrenhof JW. High-energy versus low-energy extracorporeal shock wave therapy for calcifying tendinitis of the shoulder: which is superior? A meta-analysis. Clin Orthop Rel Res. 2014;472:2816-2825.

19. Cook JL, Purdam CR. Is tendon pathology a continuum? A pathology model to explain the clinical presentation of load-induced tendinopathy. Br J Sports Med. 2009;43:409-416.

20. Yamaguchi K, Ditsios K, Middleton WD, Hildebolt CF, Galatz LM, Teefey SA. The demographic and morphological features of rotator cuff disease. A comparison of asymptomatic and symptomatic shoulders. J Bone Joint Surg Am. 2006;88:1699-1704. 
21. McFarland EG, Maffulli N, Del Buono A, Murrell GA, Garzon-Muvdi J, Petersen SA. Impingement is not impingement: the case for calling it "Rotator Cuff Disease". Muscles Ligaments Tendons J. 2013;3: 196-200.

22. Dean BJ, Gwilym SE, Carr AJ. Why does my shoulder hurt? A review of the neuroanatomical and biochemical basis of shoulder pain. Br J Sports Med. 2013;47:1095-1104.

23. Oliva F, Via AG, Maffulli N. Physiopathology of intratendinous calcific deposition. BMC Med. 2012;10:95.

24. Cools AM, Struyf F, De Mey K, Maenhout A, Castelein B, Cagnie B. Rehabilitation of scapular dyskinesis: from the office worker to the elite overhead athlete. Br J Sports Med. 2014;48:692-697.

25. Kibler WB, Sciascia A. Current concepts: scapular dyskinesis.. Br J Sports Med. 2010;44:300-305.

26. Bureau NJ. Calcific tendinopathy of the shoulder. Semin Musculoskelet Radiol. 2013;17:80-84.

27. Gosens T, Hofstee DJ. Calcifying tendinitis of the shoulder: advances in imaging and management. Curr Rheumatol Rep. 2009;11:129-134.

28. de Witte PB, de Groot JH, van Zwet EW, et al. Communication breakdown: clinicians disagree on subacromial impingement. Med Biol Eng Comput. 2014;52:221-231.

29. Schellingerhout JM, Verhagen AP, Thomas S, Koes BW. Lack of uniformity in diagnostic labeling of shoulder pain: time for a different approach. Man Ther. 2008;13:478-483.

30. Diercks R, Bron C, Dorrestijn $\mathrm{O}$, et al. Guideline for diagnosis and treatment of subacromial pain syndrome. Acta Orthop. 2014;85:314-322.

31. Andersson IH. The course of non-malignant chronic pain: a 12-year follow-up of a cohort from the general population. Eur J Pain. 2004;8:47-53.

32. Luime JJ, Kuiper JI, Koes BW, Verhaar JAN, Miedema HS, Burdorf A. Work-related risk factors for the incidence and recurrence of shoulder and neck complaints among nursing-home and elderly-care workers. Scand J Work Environ Health. 2004;30:279-286.

33. Macfarlane GJ, Hunt IM, Silman AJ. Predictors of chronic shoulder pain: a population based prospective study. J Rheumatol. 1998;25:1612-1615.

34. Kuijpers T, van Tulder MW, van der Heijden GJMG, Bouter LM, van der Windt DIAWM. Costs of shoulder pain in primary care consulters: a prospective cohort study in The Netherlands. $B M C$ Musculoskelet Disord. 2006;7:83.

35. Dinant GJ, Buntinx FF, Butler CC. The necessary shift from diagnostic to prognostic research. BMC Fam Pract. 2007;8:53.

36. Hill JC, Whitehurst DG, Lewis M, et al. Comparison of stratified primary care management for low back pain with current best practice (STarT Back): a randomised controlled trial. Lancet. 2011;378: 1560-1571.

37. Hingorani AD, Windt DA, Riley RD, et al. Prognosis research strategy (PROGRESS) 4: stratified medicine research. $B M J$. 2013;346:e5793. 
Summary 


\section{Summary}

This thesis investigates the potential role of ultrasound imaging for improved management of acute shoulder pain (pain less than 3 months) due to subacromial disorders in general practice patients. While shoulder pain is a frequently occurring complaint in general practice, treatment is not yet optimal. The course of shoulder pain has a poor prognosis, and patients with shoulder pain generate considerable costs. Mostly a subacromial disorder is the cause in patients with shoulder pain, for example tendinopathy or tear of a rotator cuff tendon. For all specific subacromial disorders, evidence based therapies are available. However, the problem arises that as regards the diagnosis, the general practitioner (GP) relies mainly on history taking and physical examination, the result of which is often not satisfying. Consequently, GPs often start an advised treatment based on patients' signs and symptoms and not on the disorder itself. This approach is not optimal as it often appears not to be effective. Moreover, it delays the onset of an adequate treatment based on the disorder. A more directed approach is indeed possible. For that, a more reliable diagnosis has to be made as early as possible. We propose that this is achievable by adding ultrasound imaging to the management of shoulder pain. Hence, the aim of this thesis was to study the effectiveness of tailoring treatment to the ultrasound diagnosis on clinical recovery of patients with acute shoulder pain, who present in general practice; the Maastricht Ultrasound Shoulder pain Trial (MUST-study). In order to reach this aim, several other studies were required. For that purpose, we had to determine the diagnostic accuracy of ultrasound imaging for detecting subacromial disorders, and had to obtain a deeper understanding about GPs perspectives on the diagnostic work-up of patients with shoulder pain in general practice. In addition, the diagnostic yield of ultrasound imaging in patients with shoulder pain in general practice had to be studied (Chapter 1).

Chapter 2 summarizes the results of a systematic review and meta-analysis into the diagnostic accuracy of ultrasound imaging for detecting subacromial disorders in patients presenting in primary and secondary care settings. So far, only studies that have reviewed the diagnostic accuracy of ultrasound imaging for the diagnosis of rotator cuff tears have been published. Twenty-three studies were included: 22 reported on full-thickness rotator cuff tears, 15 on partial-thickness tears, 3 on subacromial bursitis and 2 on tendinopathy and calcific tendonitis, respectively. Not a single study included primary care patients. The studies addressing full-thickness tears showed an overall pooled sensitivity of 0.95 , and specificity of 0.96 . For partialthickness tears, pooled sensitivity was 0.72 , and specificity 0.93 . Statistical pooling was not possible for the other disorders. For subacromial bursitis, sensitivity ranged from 
0.79 to 0.81 , and specificity from 0.94 to 0.98 , while for tendinopathy, sensitivity ranged from 0.67 to 0.93 , and specificity from 0.88 to 1.00 . Sensitivity for calcific tendonitis was 1.00 in both studies, with specificity ranging from 0.85 to 0.98 . Based on these results, we recommend ultrasound imaging in patients for whom conservative treatment fails, to rule in or out full-thickness tears, to rule in partial-thickness tears and to a lesser extent to diagnose tendinopathy, subacromial bursitis and calcifying tendonitis. This implies that ultrasound imaging also enables GPs to establish a more specific diagnosis, as these disorders are present earlier in the course.

The diagnostic work-up of patients with shoulder pain in general practice is complex. Chapter 3 aimed to explore GPs' perspectives on the diagnostic work-up of patients with shoulder pain. Semi-structured interviews with 18 GPs showed that GPs use a variety of diagnostic classifications, and that despite recommendations in the shoulder pain guideline, many GPs prefer to establish a specific diagnosis. Moreover, GPs apply different strategies establishing a diagnosis. These differences are based on expertise and experience, but also on patient preferences. GPs apply different strategies dealing the uncertainties in the complex diagnostic process. GPs mention diagnostic imaging and interdisciplinary consultation and referral as strategies dealing these uncertainties. Currently, there appears to be little agreement if, or in which phase of shoulder pain, diagnostic ultrasound is useful or indicated. This study shows no unequivocal approach regarding the diagnostic work-up of shoulder pain, and the results do not seem to be related to the GP characteristics years of experience and number of referrals for ultrasound imaging. Based on these results we advise to investigate diagnostic classification preferences, and the role of ultrasound imaging in the diagnostic process. Moreover, we advise to train GPs in decision-making concerning diagnostic work-up and dealing corresponding uncertainties.

The frequencies of specific disorders as diagnosed with ultrasound imaging in patients with shoulder pain referred from general practice is unknown. In Chapter $\mathbf{4}$ we set out to determine the frequency of specific ultrasound diagnosed shoulder disorders in daily practice in these patients and to investigate if the disorders detected differ between specific subgroups based on age and duration of pain. With $29 \%$ calcific tendonitis was the most frequently diagnosed disorder, followed by subacromial bursitis $(12 \%)$, tendinopathy $(11 \%)$, partial-thickness tears $(11 \%)$, full-thickness tears $(8 \%)$ and ACosteoarthritis $(0.4 \%)$. In $40 \%$ of patients, no disorders were found on ultrasound. Significantly more full thickness-tears were found in the $\geq 65$ years group. 'No disorders' were found significantly more often in the $<65$ years group. The supraspinatus tendon was the most frequently affected tendon (72\%). These results imply that patients can 
be stratified into diagnostic subgroups, and more tailored treatment than currently applied is possible.

The aim of Chapter 5 was to describe the study protocol of the MUST-study. This is a pragmatic, randomised controlled trial assessing the clinical effectiveness of tailoring treatment to an ultrasound diagnosis in patients with acute shoulder pain, who present in general practice. After a qualification period of two weeks, patients who were still symptomatic with standard treatment entered the trial with a 50 week follow-up period comparing two treatment strategies for patients in whom the GP suspected a subacromial disorder: treatment tailored to ultrasound diagnosis and usual care according to the shoulder pain guideline of the Dutch College of General Practitioners. Furthermore, the methodological considerations focusing on the sample size calculation, and relation between ultrasound diagnosis and treatments are discussed.

The objectives of Chapter 6 were to prospectively assess the frequencies of specific disorders as diagnosed with ultrasound imaging in patients enrolled in the MUST-study, and to identify possible predictors of shoulder pain. In this observational study, 129 patients aged 18-65 years with acute shoulder pain in whom the family physician suspected a subacromial disorder underwent standardised ultrasound imaging performed by radiologists. Subacromial disorders were present in $81 \%$ (105/129) of the patients, and $50 \%$ (52/105) of them had multiple disorders. Calcific tendonitis was the most frequently diagnosed specific disorder $(50 \%, 65 / 129)$, followed by tendinopathy (29\%, 37/129). Age $\geq 40$ years was most strongly related to a subacromial disorder (OR 14.93, 95\% $\mathrm{Cl} 5.17$ to 43.14). In these patients, a disorder was present in $91 \%$ (96/106), while in patients aged <40 years a disorder was present in $39 \%$ (9/23). We can conclude that in nearly all patients over 40 years with acute shoulder pain, ultrasound imaging showed a subacromial disorder. Timely diagnosis of underlying disorders in these patients could optimise treatment, while additional ultrasound in patients aged $<40$ years is less beneficial as the majority of those patients has no subacromial disorder, which needs confirmation in clinical studies.

Chapter 7 presents the results of the MUST-study. Over a 38-month recruitment period, 129 patients were enrolled in this trial by 26 GP practices. Eighteen patients recovered during the two-week qualification period, resulting in 111 randomised patients; 56 were allocated to ultrasound tailored treatment and 55 to usual care. Although more patients in the ultrasound tailored treated group perceived to be recovered after 1 year according to the patients' Global Perceived Effect assessment $(72.5 \%(37 / 51)$ vs. $60 \%(30 / 50)$, Odds Ratio $1.86,(95 \% \mathrm{Cl} 0.79$ to 4.36$))$, this difference 
was not significant $(p=0.15)$. Logistic mixed model analysis adjusting for age (cut-off at 50 years) and variation at the level of the GP practice, patient, and repeated observations with data from four time points $(3,6,9$ and 12 months) showed similar results (Odds Ratio $2.24,95 \% \mathrm{Cl} 0.72$ to $6.89, \mathrm{p}=0.16$ ) for patient perceived recovery after 1 year. Secondary outcome measures also showed no significant differences after 1 year.

We did not find any significant differences in the proportion of patients referred to physiotherapy (59\% of patients receiving ultrasound tailored treatment vs. $64 \%$ of patients receiving usual care), corticosteroid injections ( $39 \%$ vs. $31 \%$ ) or referrals to secondary care ( $20 \%$ vs. $13 \%)$. Our results suggest that ultrasound tailored treatment does not significantly improve patient perceived recovery after 1 year. However, it does not give a conclusive answer to whether ultrasound tailored treatment improves the clinical outcome, as the trial was under-enrolled. Furthermore, this treatment does not influence healthcare use at the study group level.

Finally, in Chapter $\mathbf{8}$ the main methodological challenges are addressed, as well as the main findings of the studies and their implications for daily practice and for future research. One of the main challenges we encountered was the recruitment of the aimed number of patients in the multicentre randomised controlled trial. Unfortunately, this challenge was not met; our study was under-enrolled. In this chapter we reflect on the ways we dealt with this challenge, and we present some lessons learned. Furthermore, the findings of our studies implicate that the management of patients with shoulder pain can be improved. Despite the fact that ultrasound imaging enables GPs to tailor treatment, ultrasound tailored treatment of patients with acute shoulder pain due to a subacromial disorder and without previous treatment, does not significantly improve patient perceived recovery after 1 year. However, the results of our trial do not give a conclusive answer to whether ultrasound tailored treatment improves outcome, as the trial was under-enrolled. From a clinical point of view, GPs should be informed that ultrasound imaging in patients with acute shoulder pain does not provide additional information to improve patient outcome. However, we can conclude that ultrasound imaging allows for stratified medicine. Therefore, future research in general practice should focus on subgroups of patients rather than a heterogeneous group of patients with a variety of underlying disorders. 
Samenvatting 


\section{Samenvatting}

Schouderpijn is een veelvoorkomende klacht in de huisartspraktijk, die het gevolg kan zijn van diverse aandoeningen. Het ziekteverloop heeft een slechte prognose en patiënten met schouderpijn zorgen voor aanzienlijke kosten. Meestal is er sprake van een subacromiale aandoening, zoals een tendinopathie of ruptuur van een rotator cuff pees. Voor elk van deze specifieke aandoeningen zijn behandelingen beschikbaar. Het probleem doet zich echter voor dat de huisarts voor het diagnosticeren van de specifieke aandoening slechts gebruik kan maken van de anamnese en het doen van lichamelijk onderzoek, en dat is in de praktijk vaak onvoldoende. Dientengevolge start de huisarts veelal een behandeling die is gericht op de pijnklachten en niet op de onderliggende oorzaak van de pijn. Deze aanpak is niet optimaal, omdat hij vaak niet afdoende blijkt te zijn. Bovendien zorgt het voor uitstel van een adequate behandeling, die wel gericht is op de onderliggende oorzaak. Toch is een meer gerichte behandeling wel degelijk mogelijk. Daartoe moet in een zo vroeg mogelijk stadium een meer betrouwbare diagnose worden gesteld. Wij veronderstellen dat dit mogelijk is door echografie op te nemen in het diagnostisch proces. Het doel van dit promotieonderzoek was om de klinische effectiviteit te bepalen van "behandeling op maat" van patiënten met acute schouder pijn in de huisartspraktijk, door de behandeling te richten op de echografie diagnose. Om dit doel te bereiken moesten er ook een aantal andere studies worden uitgevoerd. We hebben getracht om de diagnostische waarde van echografie voor de verschillende subacromiale aandoeningen vast te stellen en inzicht te krijgen in het perspectief van huisartsen op het diagnostisch proces bij patiënten met een pijnlijke schouder. Tevens hebben we de diagnostische opbrengst van echografieën van de schouder onderzocht (Hoofdstuk 1).

In hoofdstuk 2 wordt een overzicht met meta-analyse gepresenteerd van de beschikbare literatuur met betrekking tot de diagnostische waarde van echografie bij subacromiale aandoeningen voor de patiëntenpopulatie uit de huisartspraktijk en het ziekenhuis. Drieëntwintig studies werden geïncludeerd: 22 met betrekking tot volledige-dikte rupturen, 15 met betrekking tot partiële-dikte rupturen, 3 met betrekking tot subacromiale bursitis, 2 met betrekking tot tendinopathie en 2 met betrekking tot tendinitis calcarea. Geen enkel onderzoek werd uitgevoerd in de huisartspraktijk. De resultaten toonden dat de gepoolde sensitiviteit en specificiteit van echografie voor volledige-dikte rupturen respectievelijk $95 \%$ en $96 \%$ bedroeg. Voor partiële-dikte rupturen waren de gepoolde sensitiviteit en specificiteit respectievelijk $72 \%$ en $93 \%$. Statistische pooling was niet mogelijk voor de andere aandoeningen. Voor subacromiale bursitis varieerde de sensitiviteit van 79 tot $81 \%$ en de specificiteit van 
94 tot $98 \%$. Voor tendinopathie varieerde de sensitiviteit van 67 tot $93 \%$ en de specificiteit van 88 tot $100 \%$. De sensitiviteit van echografie voor tendinitis calcarea bedroeg $100 \%$ en de specificiteit varieerde van 85 tot $98 \%$. Gebaseerd op deze uitkomsten adviseren wij bij patiënten met persisterende klachten een echografie te maken om volledige-dikte rupturen aan te tonen dan wel uit te sluiten en een partiëledikte ruptuur aan te tonen. Voor het aantonen en uitsluiten van tendinopathie, subacromiale bursitis en tendinitis calcarea lijkt echografie ook geschikt. Omdat de subacromiale aandoeningen ook eerder in het beloop aanwezig zijn en dus echografisch zijn vast te stellen, kunnen huisartsen de echografie resultaten gebruiken bij het opstellen van een behandelplan.

Hoofdstuk 3 beschrijft het perspectief van huisartsen op het diagnostisch proces bij patiënten met een pijnlijke schouder. Uit semigestructureerde interviews met 18 huisartsen blijkt dat huisartsen gebruik maken van een variëteit aan diagnostische classificaties en dat, ondanks aanbevelingen in de NHG-Standaard Schouderklachten, veel huisartsen toch graag een specifieke diagnose stellen. Daarnaast verschillen huisartsen onderling in de gehanteerde strategie om tot een diagnose te komen. Deze verschillen zijn gebaseerd op kennis en persoonlijke ervaring, maar ook op voorkeuren van patiënten. Het diagnostisch proces bij schouderpijn blijkt complex te zijn en huisartsen gaan hier verschillend mee om. Het aanvragen van beeldvormende diagnostiek en intercollegiale consultatie of verwijzing werden genoemd om meer grip te krijgen op deze complexiteit. Er was weinig overeenstemming over de plaatsbepaling van echografie. De verschillen in aanpak tussen huisartsen was niet gerelateerd aan het aantal jaar werkervaring en de hoeveelheid echografieën die jaarlijks worden aangevraagd. Gebaseerd op deze resultaten adviseren wij onderzoek te doen naar de voorkeur van diagnostische classificatie en de plaatsbepaling van echografie in het diagnostisch proces. Daarnaast adviseren wij ook om huisartsen te trainen in besluitvorming rondom diagnostiek en het omgaan met bijbehorende onzekerheden.

In hoofdstuk 4 wordt retrospectief de diagnostische opbrengst beschreven van echografieën aangevraagd door huisartsen bij patiënten met een pijnlijke schouder. Met $29 \%$ was tendinitis calcarea de meest voorkomende subacromiale aandoening. Subacromiale bursitis kwam voor bij $12 \%$ van de patiënten, terwijl tendinopathie en partiële-dikte rupturen met $11 \%$ even vaak voorkwamen. Artrose van het AC-gewricht werd vastgesteld bij $0.4 \%$ van de patiënten. Bij $40 \%$ procent van de patiënten werden "geen echografische afwijkingen" geconstateerd. Volledige dikte-rupturen werden significant vaker gediagnosticeerd bij patiënten van 65 jaar en ouder, terwijl "geen echografische afwijkingen" significant vaker voorkwam bij patiënten jonger dan 65 jaar. 
De meeste aandoeningen kwamen voor in de supraspinatuspees. Op basis van deze bevindingen kan geconcludeerd worden dat patiënten met schouderpijn ingedeeld kunnen worden in diagnostische subgroepen, waardoor er meer gericht behandeld kan worden dan momenteel geadviseerd in de richtlijnen.

De onderzoeksopzet van de MUST-studie wordt beschreven in hoofdstuk $\mathbf{5}$. Dit is een pragmatische, gerandomiseerde interventiestudie naar het klinische effect van "behandeling op maat" van patiënten met acute schouder pijn in de huisartspraktijk, door de behandeling te richten op de echografie diagnose. Patiënten, die na twee weken standaard behandeling niet genezen waren, werden door loting verdeeld over de twee studiegroepen: behandeling op basis van de echografie diagnose (interventiegroep) en de gebruikelijke zorg volgens de NHG-standaard (controlegroep). De patiënten werden een jaar gevolgd. In dit hoofdstuk beschrijven we verder de methodologische overwegingen gerelateerd aan de steekproefgrootte en de koppeling tussen echografie diagnosen en behandelstrategie.

De frequentie van de echografisch vastgestelde aandoeningen in de MUST-studie en het identificeren van mogelijke voorspellers voor een aandoening wordt beschreven in hoofdstuk 6. In deze prospectieve observationele studie werd bij 129 patiënten met een leeftijd tussen 18 en 65 jaar door een radioloog een gestandaardiseerde echografie gemaakt. Bij $81 \%(105 / 129)$ van de patiënten kon een subacromiale aandoening worden vastgesteld en bij de helft hiervan (52/105) werden minimaal twee aandoeningen gezien. Tendinitis calcarea was de meest voorkomende aandoening (50\%, 65/129), gevolgd door tendinopathie (29\%, 37/129). Met een Odds Ratio van 14.93 (95\% BI 5.17-43.14) bleek leeftijd $\geq 40$ jaar de belangrijkste voorspeller te zijn voor het hebben van een subacromiale aandoening. Meer dan 90\% (96/106) van de patiënten ouder dan 40 jaar had een subacromiale aandoening, terwijl bij patiënten jonger dan 40 jaar dit percentage 39\% (9/23) was. Op basis van deze bevindingen kunnen we concluderen dat de meeste patiënten met schouderpijn, die ouder zijn dan 40 jaar, een subacromiale aandoening hebben. Bij deze leeftijdsgroep lijkt behandeling gericht op de onderliggende oorzaak van de schouderpijn mogelijk, terwijl echografie bij patiënten jonger dan 40 jaar minder voordelen oplevert, omdat de meerderheid in deze leeftijdsgroep geen subacromiale aandoening heeft is. De klinische effectiviteit hiervan moet nog worden vastgesteld.

In hoofdstuk 7 presenteren we de resultaten van de MUST-studie. Er werden 129 proefpersonen geïncludeerd, waarvan er na twee weken 18 waren genezen. De overige 111 proefpersonen werden gerandomiseerd en gelijk verdeeld over de twee 
studiegroepen: 56 in de groep met behandeling op basis van de echografie diagnose (interventiegroep) en 55 in de groep die de gebruikelijke zorg volgens de NHGStandaard kreeg (controlegroep). Hoewel er na één jaar meer patiënten in de interventiegroep waren genezen dan in de controle groep (72.5\% vs. $60 \%)$, was dit verschil niet significant (Odds Ratio 1.86, (95\% BI 0.79-4.36), p=0.15). De logistische mixed model analyse, die corrigeert voor leeftijd (cutoff 50 jaar) en variatie op het niveau van de huisartspraktijk, de patiënt en herhaalde metingen met data van vier tijdstippen (3, 6, 9 en 12 maanden) liet hetzelfde resultaat zien (Odds Ratio 2.24, 95\% BI 0.72-6.89, $p=0.16$ ) voor het door de patiënt ervaren herstel na 1 jaar. Ook voor de secundaire uitkomstmaten werden geen significantie verschillen gevonden. Er werden tevens geen verschillen gevonden tussen de percentages patiënten die verwezen zijn voor fysiotherapie ( $59 \%$ vs. $64 \%$ ), verwezen zijn naar een specialist ( $20 \%$ vs. $13 \%$ ) of een corticosteroïd injecties kregen (39\% vs. $31 \%$ ). De resultaten van deze trial suggereren dat een behandeling op basis van een echografie diagnose het door de patiënt ervaren herstel na 1 jaar niet verbetert. Echter, een definitief antwoord kunnen we niet geven, omdat er te weinig patiënten waren geïncludeerd.

In Hoofdstuk 8 beschrijven we de belangrijkste methodologische uitdaging en reflecteren we op de onderzoeksbevindingen. Tevens presenteren we de implicaties van onze studies voor de dagelijkse praktijk en voor toekomstig wetenschappelijk onderzoek. De belangrijkste methodologische uitdaging was het includeren van voldoende patiënten in de gerandomiseerde interventiestudie. Helaas is het niet gelukt om het benodigd aantal patiënten te includeren. In dit hoofdstuk bespreken we hoe we met deze uitdaging zijn omgegaan en wat we hiervan hebben geleerd. Uit onze resultaten blijkt dat de behandeling van patiënten met schouderpijn kan worden verbeterd. Ondanks het feit dat echografie bijdraagt aan een meer gerichte behandeling, suggereren de resultaten van onze trial, dat een behandeling op basis van een echografie diagnose bij patiënten met acute schouderpijn het door de patiënt ervaren herstel na 1 jaar niet verbetert. Daarom is het vanuit een klinisch perspectief raadzaam, dat huisartsen geen echografieën aanvragen bij patiënten met acute schouderpijn. Desalniettemin kunnen we concluderen, dat echografie het mogelijk maakt om gerichter te behandelen. Daarom zal toekomstig onderzoek zich moeten richten op subgroepen van patiënten met schouderpijn en niet langer op de heterogene groep van patiënten met een variëteit aan aandoeningen van de schouder. 
Valorisation 


\section{Valorisation}

Valorisation is the process of creating value out of knowledge, by making this knowledge suitable and available for economic or societal utilisation and to translate this knowledge into products, services, processes and new business. Valorisation should bring science closer to society, and has become a new maxim of modern research. ${ }^{1}$ Clearly, the scientific findings presented in this thesis have societal value. The specific societal value is discussed within their respective chapters. In this valorisation section we summarise the overall implications of the major findings and provide additional information how our scientific findings can be transferred to and utilised in practice. The societal value will be reflected on from four different perspectives: relevance for practice; target groups to whom the findings are relevant; translation of the findings in concrete activities and products; planning of the valorisation.

\section{Relevance for practice}

Accurate diagnosis and timely treatment of patients with shoulder pain seems necessary to prevent the development of a potential chronic disorder with subsequent high costs. Musculoskeletal disorders are the second most expensive disease group for healthcare costs in the Netherlands. ${ }^{2}$ In 2011 total healthcare costs for musculoskeletal disorders in the Netherlands was $€ 5.2$ billion, which was approximately $5.8 \%$ of total healthcare costs, and $€ 1.1$ billion (21\%) can be attributed to primary care. ${ }^{3}$ It is not known which part can be attributed to shoulder pain. Although shoulder pain related costs during six months after presentation do not seem alarmingly high, approximately $€ 700$ per patient, it is suggested that prolonged and recurrent episodes generate additional costs for expensive care and sick leave. ${ }^{4}$ In general, costs due to sick leave (productivity losses) are substantial and represent a higher burden to the economy than healthcare costs. ${ }^{5}$

Unfortunately, accurate diagnosis of shoulder pain is difficult because findings from medical history and physical examination often poorly correlate with the underlying disorder. $^{6-13}$ The systematic review presented in Chapter 2 shows that ultrasound imaging is accurate in diagnosing subacromial disorders in patients seen in secondary care settings for whom conservative treatment fails. This implies that ultrasound imaging enables to establish a more specific diagnosis, also in general practice patients as these disorders are present earlier in the course. The relevance of our findings are addressed for both settings. 


\section{General practice}

As discussed in Chapter 7, the results of our trial indicate that general practitioners (GPs) should refrain from ordering ultrasound imaging in patients with acute shoulder pain without previous treatment as it does not improve prognosis after one year. However, as shown in Chapters 3 and 4, ultrasound imaging is frequently applied in patients with acute shoulder pain. ${ }^{14,15}$ The results of our trial imply that the total number of ultrasound examinations can be reduced. From a healthcare perspective, this reduction will save costs. On the other hand, an economic evaluation performed from a societal perspective, which implies that all relevant costs are taken into account (for example healthcare costs and costs due to sick leave), regardless of who pays for them, will be more informative. We anticipate an economic evaluation of our trial to follow, in which analyses will be performed from a societal perspective, meaning that healthcare costs, patient and family costs, as well as productivity losses will be included.

In patients with therapy resistance, ultrasound imaging may yield a more specific diagnosis, provide a rationale for further treatment, and inform patients about the prognosis of their disorder. Two examples are given to show the benefit of ultrasound imaging in patients with persistent pain. First, calcific tendonitis is the most prevalent observed disorder in patients with shoulder pain (Chapters 4 and 6). ${ }^{15,16}$ In these cases, GPs can inform patients about the natural course, which is considered to be selflimiting with recurrent painful episodes. The self-limiting nature of this disorder may be reassuring for patients, and might prevent further treatment. If painful episodes are bothersome, treatment choice should be guided by shared decision-making based on information as to both the effectiveness and adverse events of each treatment option. Secondly, ultrasound imaging prevents unnecessary referrals to secondary care, as it enables GPs to refer patients targeted to secondary care. Preferably, patients are referred to an orthopedic surgeon in case surgery is needed, for example in case of fullthickness tendon tears. However, Chapters 4 and 6 show that full-thickness tendon tears are infrequently present in patients with acute and chronic shoulder pain, who present in general practice. ${ }^{15}$

\section{Secondary care}

Management of shoulder pain in secondary care involves various medical disciplines, for example orthopedic surgery, rheumatology, sports medicine, rehabilitation medicine, and radiology. Also for each of these disciplines the diagnosis of patients with shoulder pain is a complex problem. ${ }^{6-12}$ Especially in secondary care, an accurate 
diagnosis is essential to ensure that patients receive appropriate and timely treatment and correct information regarding their prognosis.

The results of the systematic review provided in Chapter 2 suggest that ultrasound imaging can have a positive impact on establishing a more accurate diagnosis in patients with shoulder complaints seen in a secondary care setting. Magnetic resonance imaging (MRI) and magnetic resonance arthrography (MR arthrography) can also be used to evaluate the painful shoulder. In addition, the results of Chapter 2 confirm the evidence that ultrasound imaging offers at least similar performance to $M R I$ in diagnosing full-thickness tears. Evidence has shown that ultrasound imaging is the more cost-effective test in secondary care to diagnose full- and partial thickness tears. ${ }^{6,17}$ This implies that ultrasound imaging can be the method of choice in evaluating patients with shoulder pain, and MRI can be reserved for diagnosing concomitant abnormalities in those identified for surgery, or for those patients with suspected intraarticular disorders, for example labrum lesions. ${ }^{18} \mathrm{MR}$ arthrography is more accurate in ruling out a partial-thickness tear. ${ }^{17}$ This implies that MR arthrography can be performed in cases in which ultrasound imaging is not definitive. The combination of medical history, physical examination and ultrasound imaging fulfil the need for diagnostic certainty and enables physicians to tailor treatment in most cases. For example, ultrasound imaging is able to rule in and rule out full-thickness tears and hence allows for rapid identification of those eligible to cuff repair surgery.

An example of how ultrasound imaging affects diagnosis and timely treatment is provided in a study by Rutten et al. ${ }^{19}$ This secondary care study showed that patients with posttraumatic shoulder pain have a high prevalence of unsuspected and initially missed rotator cuff tears, and ultrasound imaging was accurate in the detection of clinically relevant trauma-related shoulder disorders. ${ }^{19}$ In this study, ultrasound imaging changed the initial working diagnosis in $74 \%$ of patients with posttraumatic pain and the treatment strategy in more than half of the patients. The authors conclude that active referral for ultrasound imaging may identify these abnormalities in an earlier phase and that this might improve clinical outcome.

\section{Target groups}

The results presented in this thesis are of relevance for several target groups. First of all, GPs and their patients directly benefit from the findings from this thesis. In patients with acute shoulder pain, ultrasound imaging does not improve recovery after one year. So, GPs should follow the advice stated in the shoulder pain guidelines issued by the Dutch College of General Practitioners, and should refrain from applying ultrasound imaging in patients with acute shoulder pain. ${ }^{20}$ This implies that costs can be saved for 
an individual patient. In The Netherlands all patients have healthcare insurance, however in 2015, they have a mandatory deductible of $€ 375$. An ultrasound exam costs about $€ 80$, which therefore can be saved.

Secondly, the results are relevant to other professionals who are involved in patients with shoulder pain. The benefits for secondary care disciplines are outlined above. Also, physiotherapists can benefit from these results. In Chapter 3 we showed that GPs refer patients to physiotherapists to establish a diagnosis. ${ }^{14}$ Another study showed that twothirds of patients referred by a GP was given the referral during the first GP consultation without further treatment by the GP. ${ }^{21}$ These findings imply that GPs and patients have confidence in the process of physiotherapists. The confidence of patients is also illustrated by the number of self-referrals, direct access, to physiotherapy. In the Netherlands, patients can access physiotherapists directly; this is known as direct access or self-referral. Of all shoulder pain patients seen in physiotherapy practice, $12 \%$ use direct access. ${ }^{21}$ Nowadays, ultrasound imaging is increasingly used by physiotherapists in The Netherlands. ${ }^{22}$

Thirdly, the results presented in Chapter 7 are relevant for diagnostic centers and radiology departments offering ultrasound imaging of the shoulder. They should inform GPs in their adherence area about the effectiveness of ultrasound imaging in patients with acute shoulder pain.

Fourthly, the findings are relevant to guideline developers. The Dutch College of General Practitioners issues guidelines for general practitioners. The most recent shoulder pain guidelines were issued in $2008 .{ }^{20}$ Based on our findings, we recommend several adjustments. In order to inform patients about the natural course of a specific disorder and evidence-based treatments, GPs require more information than is currently available in the shoulder pain guidelines. Currently, the guidelines lack information about the natural course and treatments of the specific subacromial disorders. We recommend that this should be incorporated in the update of the guidelines, especially given that ultrasound imaging is frequently ordered by GPS (Chapters 3 and 4). ${ }^{14,15}$ We also recommend the introduction of a clear and unequivocal classification of shoulder pain (Chapter 3$).{ }^{14}$ Uniform classifications are essential for the timely diagnosis and treatment of shoulder pain conditions by various healthcare professionals such as GPs, physiotherapists, orthopedic surgeons and rheumatologists. Despite the shoulder pain guidelines advising GPs to classify patients based on pain and physical examination findings, GPs use a variety of classifications. This lack of uniformity is not limited to general practice, but also exists among physiotherapists, orthopedic surgeons and researchers. ${ }^{23,24}$ In 2012, the Netherlands Orthopedic Association introduced the term "Subacromial pain syndrome (SAPS)" in the eponymous "Guideline for diagnosis and treatment of subacromial pain syndrome". ${ }^{25}$ We therefore 
recommend the use of the term SAPS in the shoulder pain guidelines of the Dutch College of General Practitioners, especially considering the guidelines for physiotherapists, released by the Royal Dutch Society for Physical Therapy, are known as "Evidence Statement for Subacromial pain". ${ }^{20,26}$ In cases where the specific disorders are known, SAPS can be replaced by this specific disorder.

Finally, the results are of importance for providers of training for GPs. They should not focus only on the diagnostic work-up and therapeutic strategies as outlined in the guidelines, but should also address how to combine the guidelines, their own experience and expertise, and their patients' preferences in decision-making concerning diagnostic work-up (Chapter 3). As other factors influence patient's reassurance, GPs might benefit form training in tolerating diagnostic uncertainty, and the use of other reassuring strategies. Consequently, ultrasound imaging will be more adequately applied. ${ }^{14}$

\section{Activities and products}

Several activities have been performed and products have been developed, or will be developed in the near future. Chapters 2 to 7 are presented on national or international congresses. The evidence-based treatment protocol presented in Chapter 5 provides GPs a handle to cope with specific shoulder disorders, however, an update incorporating new evidence is recommended.

As outlined in the General Discussion of this thesis, we developed an evidence based flowchart to specify when a GP should advise ultrasound imaging to shoulder patients. This flowchart is incorporated in the application form released by Medical Coordination Centre Omnes (Omnes) for which GPs have to complete when ordering ultrasound exams. We anticipate an update of this flowchart to follow.

\section{Planning and realisation}

On a regional level, Omnes will be informed so that the flowchart to specify when a GP should advise ultrasound imaging to shoulder patients can be modified, and a regional training for GPs, addressing the topics described earlier, can be scheduled.

On a national level, further distribution can be realised by the Federation of Medical Coordination Centers, as Omnes is a member of this federation.

In addition, updating the shoulder pain guidelines is coordinated by the Dutch College of General Practitioners. 


\section{References}

1. De Jonge B, Louwaars N. Valorizing science: whose values? Science \& society series on convergence research. EMBO reports. 2009;10:535-539.

2. Meerding WJ, Bonneux L, Polder JJ, Koopmanschap MA, van der Maas PJ. Demographic and epidemiological determinants of healthcare costs in Netherlands: cost of illness study. BMJ. 1998;317: 111-115.

3. RIVM. Kosten van ziekten tool (Health care costs in the Netherlands 2011). Kosten van Ziekten 2011. Bilthoven: RIVM. Available at: www.kostenvanziekten.nl

4. Kuijpers T, van Tulder MW, van der Heijden GJMG, Bouter LM, van der Windt DIAWM. Costs of shoulder pain in primary care consulters: a prospective cohort study in The Netherlands. BMC Musculoskelet Disord. 2006;7:83.

5. Jensen MK, Sjogren P, Ekholm O, Rasmussen NK, Eriksen J. Identifying a long-term/chronic, non-cancer pain population using a one-dimensional verbal pain rating scale: an epidemiological study. Eur J Pain. 2004;8:145-152.

6. Dinnes J, Loveman E, Mclntyre L, Waugh N. The effectiveness of diagnostic tests for the assessment of shoulder pain due to soft tissue disorders: a systematic review. Health Technol Assess. 2003;7:1-166.

7. Hegedus EJ, Goode A, Campbell S, et al. Physical examination tests of the shoulder: a systematic review with meta-analysis of individual tests. Br J Sports Med. 2008;42:80-92.

8. Hermans J, Luime JJ, Meuffels DE, Reijman M, Simel DL, Bierma-Zeinstra SM. Does this patient with shoulder pain have rotator cuff disease?: The Rational Clinical Examination systematic review. JAMA. 2013;310:837-847.

9. Bamji AN, Erhardt CC, Price TR, Williams PL. The painful shoulder: can consultants agree? $B r J$ Rheumatol. 1996;35:1172-1174.

10. de Winter AF, Jans MP, Scholten RJ, Deville W, van Schaardenburg D, Bouter LM. Diagnostic classification of shoulder disorders: interobserver agreement and determinants of disagreement. Ann Rheum Dis. 1999;58:272-277.

11. Fischer AF, Dexter WW, eds. How evidence-based is our examination of the shoulder. London: Blackwell, BMJ Books; 2007. MacAuly D, Best TM, eds. Evidence based sports medicine.

12. Hughes PC, Taylor NF, Green RA. Most clinical tests cannot accurately diagnose rotator cuff pathology: a systematic review. Austr J Physiother. 2008;54:159-170.

13. Liesdek C, van der Windt DAWM, Koes BW, Bouter LM. Soft-tissue disorders of the shoulder. A study of inter-observer agreement between general practitioners and physiotherapists and an overview of physiotherapeutic treatment. Physiotherapy. 1997;83:12-17.

14. Ottenheijm RP, Hesselmans NJ, Kemper A, et al. GPs' perspectives on the diagnostic work-up in patients with shoulder pain: a qualitative study. J Eval Clin Pract. 2014;20:239-245.

15. Ottenheijm RP, van't Klooster IG, Starmans LM, et al. Ultrasound-diagnosed disorders in shoulder patients in daily general practice: a retrospective observational study. BMC Fam Pract. 2014;15:115.

16. Ottenheijm RP, Cals JW, Weijers R, Vanderdood K, de Bie RA, Dinant GJ. Ultrasound imaging for tailored treatment of patients with acute shoulder pain. Ann Fam Med. 2015;13:53-55.

17. de Jesus JO, Parker L, Frangos AJ, Nazarian LN. Accuracy of MRI, MR arthrography, and ultrasound in the diagnosis of rotator cuff tears: a meta-analysis. Am J Roentgenol. 2009;192:1701-1707.

18. Matava MJ, Purcell DB, Rudzki JR. Partial-thickness rotator cuff tears. Am J Sports Med. 2005;33: 1405-1417.

19. Rutten MJ, Collins JM, de Waal Malefijt MC, Kiemeney LA, Jager GJ. Unsuspected sonographic findings in patients with posttraumatic shoulder complaints. J Clin Ultrasound. 2010;38:457-465.

20. Winters JC, van der Windt DAWM, Spinnewijn WEM, et al. Shoulder pain guideline of the Dutch College of General Practitioners (in Dutch). Huisarts Wet. 2008;51:555-565.

21. Kooijman M, Swinkels I, van Dijk C, de Bakker D, Veenhof C. Patients with shoulder syndromes in general and physiotherapy practice: an observational study. BMC Musculoskelet Disord. 2013;14:128. 
22. Thoomes-de Graaf M, Scholten-Peeters GG, Duijn E, et al. Inter-professional agreement of ultrasoundbased diagnoses in patients with shoulder pain between physical therapists and radiologists in the Netherlands. Man Ther. 2014;19:478-483.

23. de Witte PB, de Groot JH, van Zwet EW, et al. Communication breakdown: clinicians disagree on subacromial impingement. Med Biol Eng Comput. 2014;52:221-231.

24. Schellingerhout JM, Verhagen AP, Thomas S, Koes BW. Lack of uniformity in diagnostic labeling of shoulder pain: time for a different approach. Man Ther. 2008;13:478-483.

25. Diercks R, Bron C, Dorrestijn O, et al. Guideline for diagnosis and treatment of subacromial pain syndrome. Acta Orthop. 2014;85:314-322.

26. Royal Dutch Society for Physical Therapy. Diagnosis and treatment of subacromial disorders (in Dutch). Available at: www.kngfrichtlijnen.nl (2011). 
List of publications 


\section{List of publications}

\section{Papers}

Ottenheijm RPG, Cals JWL, Weijers RE, Vanderdood K, de Bie RA, Dinant GJ.

Ultrasound imaging for tailored treatment of patients with acute shoulder pain.

Ann Fam Med 2015;13:53-55

Ottenheijm RPG, van 't Klooster IGM, Starmans LMM, Vanderdood K, de Bie RA, Dinant GJ, Cals JWL.

Ultrasound-diagnosed disorders in shoulder patients in daily general practice: a retrospective observational study. BMC Fam Pract 2014;15:115

Ottenheijm RPG, Hesselmans NJJM, Kemper A, Moser A, de Bie RA, Dinant GJ, Cals JWL. GPs' perspectives on the diagnostic work-up in patients with shoulder pain: a qualitative study.

J Clin Eval Pract 2014; 20:239-45

\section{Ottenheijm RPG.}

ESWT bij schouderpijn?

Huisarts Wet 2014;57:555

Thoomes-de Graaf M, Scholten-Peeters W, Duijn E, Karel Y, Van den Borne M, Beumer A, Ottenheijm RPG, Dinant GJ, Tetteroo E, Lucas C, Koes B, Verhagen A.

Inter-professional agreement of ultrasound-based diagnoses in patients with shoulder pain between physical therapists and radiologists in The Netherlands.

Man Ther 2014;19:478-83

Karel YH, Scholten-Peeters WG, Thoomes-de Graaf M, Duijn E, Ottenheijm RPG, Koes BW, Verhagen AP.

Current management and prognostic factors in physiotherapy practice for patients with shoulder pain: design of a prospective cohort study.

BMC Musculoskelet Disord 2013;14:62

\section{Ottenheijm RPG}

Echografie niet nuttig bij schouderinjecties.

Huisarts Wet 2013;56: 601 


\section{Hoogsteder P, Ottenheijm RPG}

Pijnlijke elleboog.

Huisarts Wet 2012;55:136

Jansen MJ, Brooijmans F, Geraets JJXR, Lenssen AF, Ottenheijm RPG, Penning LIF, de Bie RA.

Evidence Based Statement Subacromiale klachten.

Royal Dutch Society for Physical Therapy (2011): www.kngfrichtlijnen.nl

Ottenheijm RPG, Joore MA, Walenkamp GHIM, Weijers RE, Winkens B, Cals JWL, de Bie RA, Dinant GJ.

The Maastricht Ultrasound Shoulder pain trial (MUST): Ultrasound imaging as a diagnostic triage tool to improve management of patients with non-chronic shoulder pain in primary care.

BMC Musculoskelet Disord 2011;12:154.

Ottenheijm RPG, Jansen MJ, Staal JB, van den Bruel A, Weijers RE, de Bie RA, Dinant GJ. Accuracy of diagnostic ultrasound in patients with suspected subacromial disorders: a systematic review and meta-analysis.

Arch Phys Med Rehabil 2010;91:1616-25.

Erratum in: Arch Phys Med Rehabil 2010;91:1962-3.

Ottenheijm RPG, Zwietering PJ, Scherpbier AJJA, Metsemakers JFM.

Early student-patient contacts in general practice: an approach based on educational principles.

Med Teach 2008;30:802-8

Fogelholm M, van Marken Lichtenbelt WD, Ottenheijm RPG, Westerterp KR. Amenorrhea in ballet dancers in The Netherlands.

Med Sci Sports Exerc 1996;28:545-50

Van Marken Lichtenbelt WD, Fogelholm M, Ottenheijm RPG, Westerterp KR. Physical activity, body composition and bone density in ballet dancers.

Br J Nutr 1995;74:439-51 


\section{Oral presentations}

De effectiviteit van echografie bij acute schouderpijn.

NHG Wetenschapsdag 2015, Rotterdam.

Welke diagnostiek en behandelstrategie worden toegepast door de huisarts bij schouderklachten?

Schouder symposium Orthopedie MUMC 2013, Maastricht.

Plaatsbepaling van echografie bij traumata van het spier-skeletstelsel.

Onderzoek in Beweging 2013, Maastricht.

Echografie van de schouder in de eerstelijn.

Sporttrauma symposium Orthopedie MUMC 2012, Maastricht.

Accuracy of diagnostic ultrasound in patients with suspected subacromial disorders: a systematic review and meta-analysis.

Primary Care Musculoskeletal Research Congress 2010, Rotterdam.

Echografie bij musculoskeletale klachten in de huisartspraktijk.

NVMBR Jubileumcongres 2010, Maastricht.

Teaching and learning in the clinical setting: patient contacts on sound educational principles in undergraduate medical education.

$1^{\text {st }}$ annual meeting of GP/FM in Europe 2004, Kranjska Gora, Slovenia.

Teaching and learning in general practice: third year student-patient encounters.

$1^{\text {st }}$ annual meeting of GP/FM in Europe 2004, Kranjska Gora, Slovenia.

\section{Workshops}

Echografie van de schouder live, implementatie van het meekijk consult.

NHG Congres Kaderopleiding Bewegingsapparaat "A congress in motion" 2015, Utrecht.

Echografie van de schouder in de eerstelijn.

Schouder symposium Orthopedie MUMC 2013, Maastricht. 
Teaching and learning in primary care: from evidence to experience in a Dutch-Turkish educational partnership.

Wonca Europe 2008, Istanbul, Turkey.

Teaching and learning in the clinical setting: why and how?

Wonca Europe 2005, Kos, Greece.

\section{Poster presentations}

Ottenheijm RPG, Hesselmans NJJM, Kemper A, Moser A, de Bie RA, Dinant GJ, Cals JWL. Het perspectief van de huisarts op het diagnostisch proces van patiënten met een pijnlijke schouder.

NHG Wetenschapsdag 2013, Leiden.

Ottenheijm RPG, van 't Klooster IGM, Starmans LMM, Vanderdood K, de Bie RA, Dinant GJ, Cals JWL.

Schouderpijn in de huisartspraktijk; wat wordt gezien bij echografie?

NHG Wetenschapsdag 2012, Maastricht.

Ottenheijm RPG, Walenkamp GHIM, Weijers RE, Jong BMLA, Cals JWL, de Bie RA, Dinant GJ.

Echografische diagnostiek en doelgerichte behandeling van schouderpijn in de huisartspraktijk.

NHG Wetenschapsdag 2011, Nijmegen.

Ottenheijm RPG, Joore MA, Walenkamp GHIM, Weijers RE, Winkens B, Cals JWL, de Bie RA, Dinant GJ.

Maastricht Ultrasound Shoulder pain Trial: study protocol.

CAPHRI Research Day 2011, Maastricht.

Sieben JM, van Mameren H, van Ooij H, Staal B, Ottenheijm RPG, Portegijs PJM, de Bie RA.

Can we further specify acute "non-specific" low back pain? Screening for paraspinal compartment syndrome and erector spinae tears in acute low back pain patients.

IX International Forum on Primary Care Research on Low Back Pain 2007, Palma de Mallorca, Spain. 
Dankwoord 


\section{Dankwoord}

Met de verdediging van mijn proefschrift komt er een einde aan enerverende periode en breekt er weer een nieuwe fase aan. Terugkijkend op de afgelopen jaren kom ik tot de conclusie, dat ik veel inspirerende mensen heb ontmoet en het een leuke en leerzame periode is geweest. In 2003 werd duidelijk dat ik moest promoveren. Nu ben ik blij dat ik mocht promoveren. Destijds was het onderwerp van het proefschrift nog niet bekend, maar dat het betrekking zou hebben op het onderwijs in de huisartsgeneeskunde lag voor de hand. Echter, een promotietraject zou voor mij alleen slagen als ik mijn passie zou volgen. In 1989 ben ik geneeskunde gaan studeren met de gedachte om sport en geneeskunde te combineren. Later werd ik huisarts met de wens om me verder te profileren op het gebied van het bewegingsapparaat. Toen ik in 2006 zelf een sportblessure kreeg en er een echografie werd gemaakt, raakte ik enthousiast en gepassioneerd. De puzzelstukjes vielen op de juiste plaats en dit proefschrift is daarvan het resultaat. Dit proefschrift was nooit geschreven zonder de hulp van een grote groep fijne mensen.

Geert-Jan, in 2002 zaten we naast elkaar in het vliegtuig op weg naar het WONCA congres in Londen toen jij de vraag stelde of promoveren niet iets voor mij zou zijn. Het antwoord is gegeven. In 1996 heb ik je voor het eerst ontmoet. Je was mijn begeleider voor een buitenlandse stage huisartsgeneeskunde in Calgary, Canada. Tijdens mijn opleiding tot huisarts kwamen we elkaar weer tegen. Toen was je de begeleider van mijn wetenschappelijk onderzoek. In die periode nam ik samen met Josephine Asberg de taken waar van promovendus Rogier Hopstaken. Daarnaast interviewden wij onderzoekers over knelpunten bij het doen van onderzoek. Destijds dacht ik: "wat kan promoveren toch een ellende zijn". Ik heb je leren kennen als een inspirerende begeleider, maar vooral als iemand die altijd in mij heeft geloofd. Ook mijn promotietraject kende zijn tegenslagen, maar jij was er altijd om te helpen en de moed erin te houden. Je was een uitstekende promotor en ik hoop nog lang met je samen te mogen werken.

Rob, wij spraken elkaar voor het eerst in 2007. Mijn eerste ideeën voor een onderzoek leken niet haalbaar, waarop ik mijn koers wilde aanpassen. Het advies om daarover met jou te gaan praten was een gouden tip. In dit gesprek werd mij duidelijk dat mijn oorspronkelijk ideeën toch haalbaar konden zijn. Samen zijn we hierna de ideeën gaan uitwerken en werd je mijn promotor. Je positieve manier van coachen en er zijn op de momenten dat het erom ging zijn voor mij erg belangrijk geweest. Gelukkig heb ik met mijn gezin niet "onder de brug hoeven slapen". Een uitdrukking die je in het begin 
regelmatig gebruikte om duidelijk te maken dat we hier serieus werk van moesten maken. Gezien mijn toekomstige ambities zullen we nog regelmatig samenwerken en daar kijk ik naar uit.

Jochen, jij werd na de beginfase aan mijn promotieteam toegevoegd en dat was voor mij ideaal. Als geen ander weet je wat het is om ons werk als huisarts te combineren met het doen van onderzoek en dit in een levensfase met een jong gezin. Je had altijd tijd voor mij en we hebben zelfs bij je thuis vergaderd op je vrije dag. Je suggesties tijdens onze teambesprekingen waren helder en goed doordacht, waarbij je schrijftalent me veel voordelen heeft gebracht. "Kill your darlings" was voor mij niet altijd makkelijk, maar wel noodzakelijk. Jouw carrière en manier van coachen zijn een voorbeeld voor mij.

Job, in 2000 maakte jij voor mij de weg vrij in Geulle en hebt binnen de vakgroep een belangrijke rol gespeeld bij het faciliteren van mijn promotietraject. De twee spreekwoordelijk petten die je op had, één als collega in onze huisartspraktijk en de andere als vakgroepvoorzitter, hebben voor mij nooit een tegengesteld belang gediend. Mijn keuze om het onderwijs los te laten was voor het belang van de vakgroep spijtig, maar je hebt me daarna altijd gesteund in mijn ambities. Je was in onze huisartspraktijk een fijne collega en voor mij een rolmodel. Je vertrek uit onze huisartspraktijk was begrijpelijk, maar heb ik betreurd. We zullen nog regelmatig discussiëren over de "verschillende ballen in de lucht houden".

René, als radioloog in het Maastricht UMC was je vanaf het begin onderdeel van de projectgroep. Je constructieve feedback en kennis van zaken heb ik zeer gewaardeerd, waarbij je gevoel voor humor relativerend werkt.

Bjorn, jouw expertise als statisticus was onmisbaar tijdens mijn promotietraject. Tijdens onze momenten van overleg kon je op een heldere manier uitleg geven over statistische principes en het interpreteren van data, met name die uit de mixed models analyse.

Emeritus hoogleraar orthopedie Geert Walenkamp, Prof. dr. Danielle van der Windt en Dr. André Ament wil ik bedanken voor het meedenken tijdens het ontwikkelen van de MUST-studie. 
De leden van de beoordelingscommissie, Prof. dr. Lodewijk van Rhijn, Prof. dr. Patrick Bindels, Prof. dr. Nico van Meeteren en Dr. Matthieu Rutten wil ik bedanken voor hun bereidheid om dit proefschrift te beoordelen op de wetenschappelijke inhoud.

Karin, je hebt heel veel vragenlijsten verstuurd, data uit vragenlijsten verwerkt en nog veel meer. Zonder je hulp was ik nog lang niet klaar geweest met promoveren. Onze besprekingen waren altijd leerzaam, maar ook gezellig. Je kennis van SPSS is ongekend en zonder was ik verdwaald geraakt. Dat ik een lichte neiging tot controle heb is je niet ontgaan, maar dat ik pas op het einde doorkreeg dat roze je lievelingskleur is, daar kan ik als man niets aan doen.

Dan de afdeling radiologie van het Orbis MC (thans Zuyderland ziekenhuis, locatie Sittard-Geleen). Jullie betrokkenheid en inzet waren onmisbaar voor mijn onderzoek; alle echografieën zijn op jullie afdeling gemaakt. We hebben inhoudelijk en logistiek veel moeten afstemmen en dit ging altijd in een prettige sfeer. Bob, jij was mijn contactpersoon en telefonisch altijd bereikbaar voor vragen. Ook nu hebben we nog contact op het gebied van mijn verdere ontwikkeling als MSK echografist. Kurt, jij hebt meegeschreven aan de artikels en was de stille kracht op de achtergrond. De echografieverslagen van jullie beiden getuigen van een uitstekende kennis en zijn een voorbeeld van rapportage aan een huisarts. Funs en Mylene, jullie ondersteuning was onmisbaar. Onze periodieke voortgangsgesprekken verliepen altijd constructief en in een prettige sfeer. Fien en Monique, jullie waren voor mij de aanvoerders van het polikliniekteam en hebben geweldig werk geleverd.

Dirk, Jacqueline en Alfons, ook jullie zijn onmisbaar geweest door het ontwikkelen van de MUST webapplicatie. Het logistieke systeem was een onmisbare toepassing, wat ons veel voordeel heeft gebracht. Dirk, ik kon altijd op je terugvallen bij acute problemen en je behulpzaamheid was groot.

Ine, je bent een onmisbare schakel binnen de vakgroep. Regelmatig moest ik een beroep op je doen en meestal ging het over het maken van afspraken met mijn (co)promotoren. Samen met Conny werd er snel zaken gedaan, wat voor mij prettig werken was. Ik snoep niet, maar greep toch wel eens lekker in dat bakje met Engelse drop of spekjes.

Conny, je reageerde altijd snel op mijn vragen en verzoeken met betrekking tot het plannen van de afspraken met Rob. Je manier van reageren was altijd positief, bedankt. 
Suzanne, Frits en Judith, bij jullie kreeg ik altijd hulp bij vragen of met het versturen van brieven en vragenlijsten, bedankt voor deze steun.

Ellen, bij jou kon ik altijd binnenlopen met vragen die betrekking hadden op financiën. Je hielp me altijd snel en deskundig, bedankt.

Babette, bij jou kon ik altijd aankloppen met vragen over het academische onderzoeksveld en veel andere zaken. Ook jij hielp me altijd snel en kundig, bedankt.

Piet, je adviezen ten tijde van het indienen van het onderzoeksvoorstel bij de METC waren helder en scherp, bedankt.

Dan het MCC Omnes team. Vanaf het begin hebben jullie mijn promotietraject gesteund en gesponsord. MUST werd een begrip in de regio. Marc en Mariëlle, jullie betrokkenheid was vanaf het begin essentieel en daarnaast een grote steun voor mij. Paul, jij nam na de beginfase de taak van Marc over en hebt de ingezette lijn doorgetrokken en krachtig ondersteund. Toen we in 2013 de inclusieprocedure wilden verplaatsen naar de afdeling Radiologie is jullie steun onmisbaar geweest. Lilo, jij bent een onmisbare schakel in het Omnes team, werkt doelgericht en altijd bereid om te helpen. Ook bij ons anderhalvelijns project werkt je inbreng stimulerend. Wim, jij bent de financiële man, dacht mee indien nodig en hebt veel betalingen moeten doen. Marlou en Sandy, destijds werkzaam als directiesecretaresses, jullie hebben mij op een erg prettige manier geholpen met het drukwerk en het gereedmaken van het onderzoeksmateriaal voor de huisartsen.

De medewerkers van Andi Druk wil ik bedanken voor hun hulp bij het drukken van alle onderzoeksmaterialen en dat waren er heel veel.

Daphne Adams, nu werkzaam bij Canon Nederland, wil ik bedanken voor het maken van het MUST logo, dat al het onderzoeksmateriaal heeft gekleurd en herkenbaar maakte.

Alle deelnemende huisartsen en praktijkassistentes wil ik heel graag bedanken. Zonder jullie hulp was dit promotietraject nooit geslaagd. Velen van jullie hebben patiënten geïncludeerd en anderen zijn geïnterviewd. Het aantal patiënten dat voldeed aan de inclusie criteria viel tegen, waardoor ik langer dan beoogd een beroep op jullie heb moeten doen. Als onderzoeker voelde ik me bezwaard om jullie telkens te moeten vragen om te blijven includeren, omdat ik als huisarts weet ik wat het betekent om 
deze vraag te krijgen. Een speciale dank gaat uit naar de inclusietopscorers Tjeu Klaassen, Ruud Ubachs en de huisartsen van Overhoven-Hoogveld, Sandra van der Busse, Alfons Alberts en Kick Hamers, die respectievelijk 15, 12 en 15 patiënten hebben geïncludeerd.

Mijn medeauteurs: Mariëtte Janssen, Bart Staal, Ann van den Bruel, Albine Moser, en Manuela Joore. Mijn grote dank voor jullie hulp en ondersteuning. Een speciale dank gaat uit naar Mariëtte. Samen hebben we veel tijd gestoken in de systematic review en het schrijven van het KNGF Evidence Statement Subacromiale klachten. Tevens heb je geholpen bij de Instructiebijeenkomst voor fysiotherapeuten in september 2010, waar je samen met Jacques Geraets dit Statement hebt gepresenteerd.

Anouk, Nicolle, Inge en Laurens, ook jullie zijn medeauteurs, maar als WESP-student waren jullie ook collega onderzoeker. Jullie hebben onmisbaar werk verricht en het was een voorrecht om jullie te begeleiden.

Jacques, als fysiotherapeut ben je gepromoveerd op schouderklachten. Bij het opzetten van mijn onderzoek heeft jouw kennis me geholpen. Ook je bijdrage aan de Instructiebijeenkomst voor fysiotherapeuten in september 2010 heb ik zeer gewaardeerd.

De fysiotherapeuten, die patiënten hebben behandeld en bereid waren om patiënten te verwijzen voor inclusie, wil ik ook graag bedanken.

Tiny Wouters, je hebt een grote klus geklaard met het maken van de PDF van dit proefschrift. Het manuscript voor de beoordelingscommissie heb je snel en deskundig in elkaar gezet, wat voor mij heel prettig was. Daarna hadden we meer tijd en ik ben trots op het eindresultaat, bedankt.

Jan Klerkx and Stefanie Portelli, thank you very much for your linguistic advice, which improved the quality of my manuscripts and thesis. The advice was always knowledgeable and quick, thanks.

Mijn collega's in de huisartspraktijk: Jean, Ingrid, Marscha, Marieke, Eveline en Esther. Maar eerst begin ik met Paul en Marieke. Paul, dat jij vertrok in 2009 vond ik echt niet leuk; je was een echt maatje. Samen met Jean en Job ondersteunde je mijn ambitie om een musculoskeletale echografie opleiding te volgen en een echografie apparaat te kopen. Dat was een grote investering, waarvan het nog maar de vraag was 
of deze zich terug zou betalen. Echter, jullie visie kenmerkte zich door vooruitstrevendheid en innovatie. Daarvan pluk ik nu de vruchten. Het vertrek van Paul betekende de komst van Marieke. Marieke, tijdens je huisartsopleiding in onze praktijk heb ik je leren kennen als een fijne collega, maar vooral als een prettige persoonlijkheid. Helaas hebben we maar een paar jaar samengewerkt, omdat je verhuisde naar het oosten van het land. Je was een fijn maatje en ik vind het jammer dat we elkaar nauwelijks meer zien of spreken. Jean, mijn langst zittende collega, de rust die je uitstraalt en je analytisch vermogen zijn eigenschappen die ik bewonder. Ook jij hebt een terugtrekkende beweging gemaakt, omdat er andere uitdagingen op je pad kwamen, waarbij jouw kwaliteiten goed tot hun recht komen. Ingrid, toen ook Marieke vertrok was jij het lot uit de loterij. Na een aantal bewogen jaren, was er behoefte aan rust en stabiliteit. Jouw komst heeft hiervoor gezorgd. Het is heel prettig om met je samen te werken. Op het moment dat Job uit de praktijk wegging brak voor mij een drukke fase in het onderzoek aan, waardoor ik een halve dag per week extra afwezig was. Jouw steun was onmisbaar en erg prettig om te ervaren; ook jij bent voor mij het voorbeeld van een maatje. Marscha, voor jou kwam er een plaats in onze praktijk door het vertrek van Job. Jouw manier van werken getuigd van een grote betrokkenheid bij de patiënt en huisartspraktijk. Kwaliteiten waar onze praktijk zijn voordeel mee kan doen. Ook hebben we al mogen genieten van je muzikale talenten en ik hoop dat dit nog lang mag duren. Dan onze praktijkassistentes, Marieke, Eveline en Esther, in volgorde van langst werkende. Jullie zorgen voor een leuke en onmisbare dynamiek in onze praktijk, hoewel we ook een lastige periode samen hebben doorgemaakt. Verschillen zullen er altijd zijn en deze kunnen elkaar goed aanvullen. De kijk die jullie op het leven hebben werkt voor mij verhelderend.

Dan mijn collega's van de hagro Elsloo-Geulle: Jos, Loes, Suzanne, Petra, Henk (inmiddels met pensioen), Hans en Fianna. Onze praktijken hebben een lange traditie van samenwerken. Hoewel er ook verschillen zijn, lukt het ons om op steeds meer gebieden samen te werken met het doel de zorg voor patiënten te verbeteren. Jullie hebben mijn promotietraject op een prettige manier ondersteund door patiënten te includeren. Daarnaast ondersteunen jullie mijn werk als kaderhuisarts bewegingsapparaat met het verwijzen van patiënten en het kritisch meedenken met het verder ontwikkelen van de anderhalvelijnszorg (het meekijkconsult). Ik hoop nog lang met jullie te mogen samenwerken.

Rychard en Paul, wat vond ik de opleiding tot MSK echografist bij Fysus toch leuk en leerzaam. Rychard, als geen ander kun jij mensen prikkelen met opmerkingen en vragen. Het heeft mij aan het denken gezet, wat heeft geleid tot de aanzet van dit 
proefschrift. Daarnaast ben je een expert bij uitstek en ik hoop nog veel van je te mogen leren. De afspraak om weer een keer te komen kijken moet ik nog maken, maar zal niet lang meer op zich laten wachten. Paul, ook jij hebt veel expertise en een prettig gevoel voor humor, wat de masterclass bijeenkomsten naast leerzaam ook nog eens leuk maakten. Ik heb wel weer zin een kroketje.

Petra en Jan van Dynamic, jullie ondersteuning met echografie apparatuur in mijn praktijk is vanaf het begin onmisbaar geweest voor mijn ontwikkeling tot MSK echografist. Deskundig en betrouwbaar zijn kernwoorden die jullie bedrijf typeren, maar bovenal zijn jullie erg prettig om mee samen te werken.

Patrick, we hebben samen de MSK echografie opleiding gedaan. Uren hebben we in de auto gezeten op weg naar Baarn en Rotterdam, zodat we tijd hadden om verhalen te vertellen ons werk en privé leven. Daarnaast werken we ook prettig samen in het gezondheidscentrum. Je expertise als fysiotherapeut is enorm, waarvan ik nog lang hoop te profiteren.

Randy, we leerden elkaar kennen op de tennisbaan, waar je de ballen soms om mijn oren sloeg. Al snel troffen we elkaar regelmatig om ideeën uit te wisselen, jij als fysiotherapeut en ik als huisarts. Met bewondering heb ik gezien hoe je een eigen praktijk hebt opgezet en hiermee bent gegroeid. Het eerste stapje op gebied van samenwerking is inmiddels gemaakt en wie weet wat er nog in het vat zit; ik heb er zin in.

Laury, we zaten samen nog op een kamer toen ik mijn eerste ideeën voor een promotietraject ging uitwerken. Onze "lunchloopjes" zijn altijd gezellig, maar zijn ook fijne momenten om samen te reflecteren op ons werk en privé leven. Je carrière als huisarts heb je onderbroken om één bal minder in de lucht te hoeven houden, zodat je ook aandacht kon gaan besteden aan een promotietraject. Een moedige beslissing die alleen maar respect verdient.

Maurice, samen Alpe d'HuZes fietsen was een van de hoogtepunten van onze vriendschap tot nu toe. Samen met Patrick reden we zij aan zij naar boven, waarbij opgeven geen optie was. Die dagen in de Franse Alpen, samen met Iris en anderen, waren bijzonder en vormend. Dat we nog veel hoogtepunten samen mogen beleven. We fietsen nog elke zondagochtend met Harold, onze koempel, en noemen ons de "Goed weer fietsers", wat denk ik meer op ons humeur dan op het weer slaat. Met ons drieën wielrennen we over de heuvels en onderweg praten we over voetbal en andere 
belangrijke zaken. Heerlijk om samen te doen. De inspanningen en ervaringen van onze zonen op de voetbalvelden geven veel voer voor discussie. Wie weet staan ze ooit nog samen in één elftal, maar dan moet dat wel Oranje zijn, want sjengen en koempels in één elftal in Limburg lijkt nog ver weg. Op de fiets gaan we heuvel op en af, waarbij er maar één het snelste boven kan zijn.

Papa en mama, altijd staan jullie klaar om te helpen. Van jullie heb ik alle ruimte gekregen om me te ontwikkelen. De steun, interesse en het vertrouwen in mij voelen aan als een warm bad. Het advies om mijn passies te volgen probeer ik ook mijn kinderen mee te geven. Papa, je adviezen tijdens mijn promotietraject werkten verhelderend. Tijdens mijn middelbareschooltijd zei je vaker tegen mij: "we zijn niet lui en schrijven alles op". Ik heb geschreven. Mama, jouw rol in ons gezin is altijd ondersteunend geweest, waarbij de volgende quote van Thich Nhat Hanh aangeeft wie jij bent: "We really have to understand the person we want to love. If our love is only a will to possess, it is not love. If we only think of ourselves, if we know only our own needs and ignore the needs of the other person, we cannot love". Ik kan nog veel van jullie leren.

Mijn broers Stefan en Coen, wat vind ik het leuk dat jullie mijn paranimfen zijn. We hebben veel gezamenlijke interesses, wat het altijd weer gezellig maakt als we elkaar zien. Onze banen hebben veel raakvlakken, zodat ik ook daarvan veel van jullie kan leren. We hebben allemaal onze passie gevonden en gaan ervoor, ieder op zijn eigen manier. De levensweg die we afleggen levert interessante en diepgaande discussies op. We zijn er nog niet. Mijn schoonzussen Anne-Margreet en Beatrijs, jullie zijn geweldige partners voor mijn broers en erg prettig om in de familie te hebben. Ondanks dat we verspreid over het land wonen, hebben onze kinderen het samen fijn en kijken ze telkens weer uit om elkaar te zien. Laten we vaker samenkomen.

Mijn schoonfamilie, Hein en Marlies, Marc, Bas en Vera, jullie zijn gastvrij en staan altijd klaar om te helpen. Het oppassen door "opa en oma" op de kinderen gaf Miriam en mij de ruimte om te werken en ons verder te ontwikkelen, bedankt.

Tenslotte, Miriam, Yannick en Sem. Miriam, wat is het toch fijn om jou aan mijn zijde te hebben. Samen hebben we ons gezin op de rails gezet en houden alles draaiende. Dat laatste is niet altijd eenvoudig, zeker niet nu onze jongens elke avond van de week moeten trainen. De afgelopen jaren heb je me alle ruimte gegeven om dit promotietraject tot een goed einde te brengen. Regelmatig was ik's avonds ook nog weg in verband met verplichtingen als huisarts. Zeker de laatste twee jaren waren 
hectisch, omdat je een masteropleiding hebt gevolgd, subsidieaanvragen moest schrijven en ook nog werkte als JGZ-arts. Innovator, ook jij hebt je passie gevonden; ga ervoor! Mijn jongens, wat is het toch leuk om jullie vader te zijn. Met bewondering kijk ik naar jullie ontwikkeling en ik geniet van het samen zijn. Voetballen is jullie passie en $\mathrm{ik}$ ben trots dat ik jullie trainer en coach heb mogen zijn. Mogelijk komen er weer andere passies en vergeet niet om er altijd vol voor te gaan, zodat je geen spijt krijgt als iets niet lukt. Jullie aanwezigheid is een leerzame spiegel. 
Curriculum vitae 


\section{Curriculum vitae}

Ramon Ottenheijm was born on May the $30^{\text {th }} 1971$ in Washington DC, USA. His parents immigrated back to the Netherlands when he was a baby. He received secondary education at the Elshof College (currently known as Kandinsky College) in Nijmegen, the Netherlands. In 1989 he attended medical school at Maastricht University, and in his third year he went to the Manchester Royal Infirmary in Manchester, United Kingdom, for an elective in orthopaedic surgery. Before enrolling the internships, he participated in a research project on ballet dancers at the department of Human Biology of Maastricht University, under the supervision of professor Wouter van Marken Lichtenbelt. After a general practice elective internship at the University of Calgary, Canada, he received his medical degree in 1996. The year after graduation, he combined working as a physician in an asylum seekers centre (Echt) with teaching medical skills at the Skillslab of Maastricht University. During his internships it became clear that he wanted to become a general practitioner to be able to be in a more personal relationship with patients and started his residency in Maastricht in 1997. In 2000 he graduated as a general practitioner and started working in an academic general practice in Geulle. From then on he is combining clinical work with teaching and research at the Department of Family Medicine of Maastricht University. After nine years being involved in teaching, he shifted his attention to a $\mathrm{PhD}$ project on ultrasound imaging in shoulder patients, under the supervision of professor Geert-Jan Dinant and Jochen Cals (Department of Family Medicine), and professor Rob de Bie (Department of Epidemiology). The idea of this PhD project originated in his general practice, where, after an extensive training program, ultrasound imaging was introduced in the management of patients with musculoskeletal complaints. He will continue his work as a general practitioner and researcher/teacher.

From 2007 till 2012, Ramon was member of the supervisory board of MCC Omnes (Diagnostic and transmural care centre), Sittard-Geleen. He is registered as a general practitioner with special interest in research (2009), and as a general practitioner with special interest in musculoskeletal disorders (2013). He works as advisor to the musculoskeletal projects of MCC Omnes.

Ramon is happily married to Miriam Weijers, and is a father to their two sons, Yannick and Sem. He enjoys sports and reading. The last 8 years he was trainer-coach of the football teams of his sons, and he was member of the youth committee of their football club. 


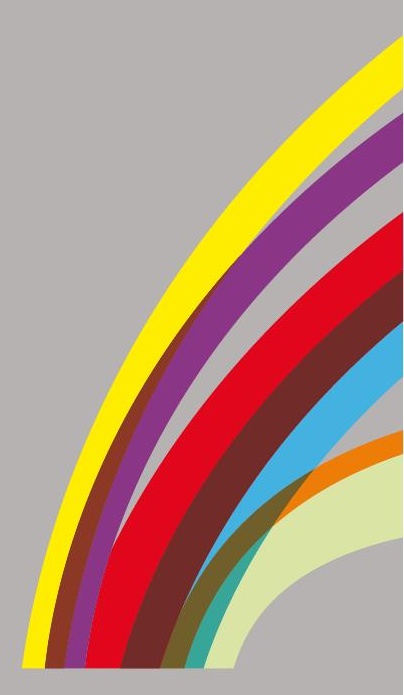

must 